\title{
Search for new phenomena in final states with large jet multiplicities and missing transverse momentum using $\sqrt{s}=13 \mathrm{TeV}$ proton-proton collisions recorded by ATLAS in Run 2 of the LHC
}

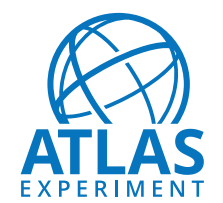

\section{The ATLAS collaboration}

E-mail: atlas.publications@cern.ch

ABSTRACT: Results of a search for new particles decaying into eight or more jets and moderate missing transverse momentum are presented. The analysis uses $139 \mathrm{fb}^{-1}$ of proton-proton collision data at $\sqrt{s}=13 \mathrm{TeV}$ collected by the ATLAS experiment at the Large Hadron Collider between 2015 and 2018. The selection rejects events containing isolated electrons or muons, and makes requirements according to the number of $b$-tagged jets and the scalar sum of masses of large-radius jets. The search extends previous analyses both in using a larger dataset and by employing improved jet and missing transverse momentum reconstruction methods which more cleanly separate signal from background processes. No evidence for physics beyond the Standard Model is found. The results are interpreted in the context of supersymmetry-inspired simplified models, significantly extending the limits on the gluino mass in those models. In particular, limits on the gluino mass are set at $2 \mathrm{TeV}$ when the lightest neutralino is nearly massless in a model assuming a two-step cascade decay via the lightest chargino and second-lightest neutralino.

KEYWORDS: Hadron-Hadron scattering (experiments)

ARXIV EPRINT: 2008.06032 


\section{Contents}

1 Introduction 1

2 ATLAS detector 2

3 Datasets 3

3.1 Data 3

3.2 Monte Carlo simulations 4

$\begin{array}{lll}4 & \text { Reconstruction and particle identification } & 7\end{array}$

$\begin{array}{llr}5 & \text { Event selection } & 9\end{array}$

6 Background estimation $\quad 11$

$\begin{array}{lll}6.1 & \text { Multijet background } & 13\end{array}$

$\begin{array}{lll}6.2 & \text { Leptonic backgrounds } & 16\end{array}$

$\begin{array}{ll}\text { 6.3 Background normalisation corrections } & 17\end{array}$

$\begin{array}{lll}6.4 & \text { Systematic uncertainties } & 18\end{array}$

$\begin{array}{lll}7 & \text { Results and interpretation } & 23\end{array}$

$\begin{array}{llr}8 & \text { Conclusion } & 28\end{array}$

$\begin{array}{ll}\text { The ATLAS collaboration } & 36\end{array}$

\section{Introduction}

The Large Hadron Collider [1] (LHC) has produced a large dataset of proton-proton ( $p p$ ) collisions at a centre-of-mass energy of $13 \mathrm{TeV}$ enabling searches for new heavy particles predicted by theories such as supersymmetry (SUSY) [2-7]. Evidence for SUSY models may be sought through searches for the production of these heavy particles (such as gluinos) decaying, often via extended cascades, into lighter ones. If the lightest of these interacts only weakly and is stable then it can be an ideal dark-matter candidate.

In $R$-parity-conserving (RPC) [8] SUSY models, the presence of a stable lightest supersymmetric particle (LSP) often leads to final states with significant missing transverse momentum $\left(E_{\mathrm{T}}^{\text {miss }}\right)$, often accompanied by a large number of jets. Large jet multiplicities would also occur in events in which gluinos decay via $R$-parity-violating (RPV) [9] couplings on short $(\lesssim n s)$ timescales. In this case the LSPs decay within the detector volume, so that the only invisible particles produced are neutrinos coming from SUSY particle decays, and hence the $E_{\mathrm{T}}^{\text {miss }}$ per event is generally smaller. A similar signature arises from any model in which cascade decays lead to the production of many jets, together with $E_{\mathrm{T}}^{\text {miss }}$ either from dark-matter particles or neutrinos. 
This paper reports the results of an analysis of $139 \mathrm{fb}^{-1}$ of $p p$ collision data recorded at $\sqrt{s}=13 \mathrm{TeV}$ by the ATLAS experiment [10] throughout the entire Run-2 period of the LHC (2015-2018). It explores events with significant $E_{\mathrm{T}}^{\mathrm{miss}}$ and at least eight jets with large transverse momentum $\left(p_{\mathrm{T}}\right)$. Selected events are further classified into categories based on the presence of jets containing $b$-hadrons ( $b$-jets) or on the sum of the masses of largeradius jets. The $b$-jet selection improves sensitivity to beyond-the-Standard-Model (BSM) signals with enhanced heavy-flavour decays. Given the unusually high jet multiplicities of the selected events, large jet masses can originate both from the capture of decay products from boosted heavy particles, including top quarks, and from accidental combinations [11]. The major backgrounds to the signal in this search are multijet production from QCD processes, top quark pair production $(t \bar{t})$ and $W$ boson production in association with jets $(W+$ jets $)$.

Previous searches by ATLAS in similar final states were carried out on smaller LHC datasets recorded at $\sqrt{s}=7 \mathrm{TeV}$ and $\sqrt{s}=8 \mathrm{TeV}$ during 2011 and 2012 [12-14]. In addition, two searches were performed at $\sqrt{s}=13 \mathrm{TeV}$, one analysing the 2015 dataset [15] and one combining it with 2016 data [16] to achieve a total integrated luminosity of $36 \mathrm{fb}^{-1}$. The current analysis extends those previous studies by including the complete Run-2 LHC dataset, by using an optimised selection tailored to the increased integrated luminosity, and also by incorporating several improved analysis methods which further increase sensitivity. One such development is the use of the particle-flow jet and $E_{\mathrm{T}}^{\mathrm{miss}}$ reconstruction algorithms recently developed for the ATLAS experiment [17]. These algorithms combine measurements of inner tracker and calorimeter energy deposits to improve the accuracy of the charged-hadron measurement, leading to improvements in the jet and $E_{\mathrm{T}}^{\mathrm{miss}}$ resolution and stability against additional $p p$ interactions in the same LHC bunch crossing. The analysis also employs an improved $E_{\mathrm{T}}^{\text {miss }}$ significance calculation [18], which accounts for the resolution of the reconstructed objects individually. This new definition increases the separation between events in which the $E_{\mathrm{T}}^{\text {miss }}$ originates from weakly interacting particles and those in which $E_{\mathrm{T}}^{\text {miss }}$ is only due to detector resolution effects. The combination of the larger dataset and the developments in the analysis methodology provides this analysis with sensitivity over a significantly increased mass range.

\section{ATLAS detector}

The ATLAS detector [10] is a multipurpose particle detector with a nearly $4 \pi$ coverage in solid angle. ${ }^{1}$ It consists of an inner tracking detector (ID) surrounded by a thin superconducting solenoid providing a $2 \mathrm{~T}$ axial magnetic field, electromagnetic (EM) and hadronic

\footnotetext{
${ }^{1}$ ATLAS uses a right-handed coordinate system with its origin at the nominal interaction point in the centre of the detector. The positive $x$-axis is defined by the direction from the interaction point to the centre of the LHC ring, with the positive $y$-axis pointing upwards, while the beam direction defines the $z$-axis. Cylindrical coordinates $(r, \phi)$ are used in the transverse plane, $\phi$ being the azimuthal angle around the $z$-axis. The pseudorapidity $\eta$ is defined in terms of the polar angle $\theta$ by $\eta=-\ln \tan (\theta / 2)$. The rapidity is defined as $y=0.5 \ln \left[\left(E+p_{z}\right) /\left(E-p_{z}\right)\right]$ where $E$ denotes the energy and $p_{z}$ is the component of the momentum along the beam direction. The transverse energy is defined to be $E_{\mathrm{T}}=E \cos \theta$. The angular distance $\Delta R$ is defined as $\sqrt{(\Delta y)^{2}+(\Delta \phi)^{2}}$.
} 
calorimeters, and a muon spectrometer. The inner tracking detector covers the pseudorapidity range $|\eta|<2.5$. It consists of silicon pixel, silicon microstrip, and transition radiation tracking detectors. A new inner pixel layer, the insertable B-layer [19, 20], was added at a mean radius of $3.3 \mathrm{~cm}$ before the start of the 2015 data-taking period, improving the identification of $b$-jets. Lead/liquid-argon (LAr) sampling calorimeters provide EM energy measurements with high granularity. A steel/scintillator-tile hadron calorimeter covers the central pseudorapidity range $(|\eta|<1.7)$. The endcap and forward regions are instrumented with LAr calorimeters for EM and hadronic energy measurements up to $|\eta|=4.9$. The muon spectrometer surrounds the calorimeters and is based on three large air-core toroidal superconducting magnets with eight coils each. The field integral of the toroids ranges between 2.0 and $6.0 \mathrm{Tm}$ across most of the detector. The muon spectrometer includes a system of precision tracking chambers and fast detectors for triggering. A two-level trigger system [21] is used to select events. The first-level trigger is implemented in hardware and uses a subset of the detector information to keep the accepted rate below $100 \mathrm{kHz}$. This is followed by a software-based trigger that reduces the accepted event rate to $1 \mathrm{kHz}$ on average.

\section{Datasets}

\subsection{Data}

The events considered in this paper correspond to $139 \mathrm{fb}^{-1}$ of $p p$ LHC collision data collected between 2015 and 2018 by the ATLAS detector, at a centre-of-mass energy of $13 \mathrm{TeV}$ and with a $25 \mathrm{~ns}$ proton bunch crossing interval. In 2015-2016 the average number of interactions per bunch crossing (pile-up) was $\langle\mu\rangle=20$, increasing to $\langle\mu\rangle=38$ in 2017 and $\langle\mu\rangle=37$ in 2018 . The uncertainty in the combined 2015-2018 integrated luminosity is $1.7 \%$ [22], obtained using the LUCID-2 detector [23] for the primary luminosity measurements.

Events were recorded using a variety of trigger selections. During both 2015 and 2016, events were selected by a trigger requiring at least six jets with $E_{\mathrm{T}}>45 \mathrm{GeV}$ and central pseudorapidity, $|\eta|<2$.4. Further events were triggered in 2015 by requiring the presence of at least five jets with $E_{\mathrm{T}}>70 \mathrm{GeV}$ and $|\eta|<3.2$, and in 2016 with a trigger requiring at least five jets with $E_{\mathrm{T}}>65 \mathrm{GeV}$ and $|\eta|<2.4$. In both 2017 and 2018, events were selected by triggers requiring at least five jets with $E_{\mathrm{T}}>70 \mathrm{GeV}$ and $|\eta|<2.4$, or seven jets with $E_{\mathrm{T}}>45 \mathrm{GeV}$ and $|\eta|<2.4$. Additional triggers were also used in 2017 and for some periods in 2018, which required at least six jets with $E_{\mathrm{T}}>45 \mathrm{GeV}$ and $|\eta|<2.4$. Due to their large trigger rates, these were set to select only a fraction of the events, approximately $15 \%$ of the two years' data; such triggers are referred to as 'prescaled' triggers. The trigger jet calibration was improved in 2017-2018, resulting in substantially improved efficiency [24] after offline selection for the same trigger thresholds. The trigger selections are summarised later in table 1 together with further event selections.

Data quality requirements are imposed to ensure that only events in which the entire ATLAS detector was functioning well are used [25]. These, for example, exclude events containing data corruption in the ID and calorimeters, excessive noise and spurious jets produced by non-collision backgrounds [26, 27]. 


\subsection{Monte Carlo simulations}

Simulated events produced with several Monte Carlo (MC) event generators are used to predict yields for subdominant background contributions from Standard Model (SM) processes and for possible signals.

All simulated events are overlaid with multiple $p p$ collisions simulated with the soft QCD processes of PYTHIA 8.186 [28] using the A3 set of tuned parameters (A3 tune) [29] and the NNPDF2.3 LO parton distribution functions (PDFs) [30]. The simulated events are required to pass the trigger selections, and are weighted such that the pile-up conditions match those of the data. The response of the detector to particles was modelled with an ATLAS detector simulation [31] based on GEANT4 [32] (full simulation), or using fast simulation based on a parameterisation of the performance of the ATLAS EM and hadronic calorimeters [33] and on GEANT4 elsewhere.

For the generation of $t \bar{t}$ and single top quarks via the $W t$ process and in the $s$-channel, matrix elements were calculated at next-to-leading order (NLO) using the PowHEGBox v2 generator [34-39] with the NNPDF3.0 NLO PDF set [40] in the five-flavour scheme. Electroweak $t$-channel single-top-quark events were generated using PowHEGBox v2, using the four-flavour scheme for the NLO matrix element calculations together with the fixed four-flavour PDF set NNPDF3.04f NLO. The diagram removal scheme [41] was used to prevent $W t$ events from being counted as $t \bar{t}$ events beyond leading order (LO). For these processes, the top quarks were decayed using MADSPIN [42, 43] preserving all spin correlations, while for all processes the parton shower, fragmentation, and the underlying event were simulated using PyTHIA 8.230 [44] with the NNPDF2.3 LO PDF set and the ATLAS A14 tune [45]. The top quark mass was set to $172.5 \mathrm{GeV}$. The $h_{\text {damp }}$ parameter, which controls the $p_{\mathrm{T}}$ of the first additional emission beyond the Born configuration in Powheg, was set to 1.5 times the mass of the top quark. The main effect of this parameter is to regulate the high- $p_{\mathrm{T}}$ emission against which the $t \bar{t}$ system recoils. The EvTGEN v1.6.0 program [46] was used to model properties of the $b$ - and $c$-hadron decays for this process and all others not simulated with SHERPA [47] unless otherwise stated. Simulated $t \bar{t}$ events are normalised to the cross-section calculated at next-to-next-to-leading order (NNLO) in perturbative QCD, including soft-gluon resummation to next-to-next-to-leading-logarithm (NNLL) accuracy [48]. The single-top-quark events for the $W t$ channel are normalised using its approximate NNLO prediction $[49,50]$, while the $t$ - and $s$-channels are normalised using their NLO predictions $[51,52]$.

Events containing $t \bar{t}$ and additional heavy particles - comprising three or four top quarks, $t \bar{t}+W, t \bar{t}+Z$ and $t \bar{t}+W W$ production - were modelled using MADGrAPH5_aMC@NLO [53] for the matrix element calculation, interfaced to the PYTHIA 8 parton shower, hadronisation and underlying event model. The $t \bar{t}+W W$, three- and four-top-quark processes were simulated at LO in the strong coupling constant $\alpha_{\mathrm{S}}$, using MadGraph5_aMC@NLO v2.2.2 interfaced to Pythia 8.186. The predicted production cross-sections were calculated to NLO as described in ref. [53] for these processes other than three-top-quark production, for which the cross-section was calculated to LO. The production of $t \bar{t}+W$ and $t \bar{t}+Z$ events was modelled using MAdGraph5_aMC@NLO v2.3.3 
at NLO with the NNPDF3.0 NLO PDF. Top quarks were decayed at LO using MADSPIN to preserve spin correlations. Parton shower and hadronisation were modelled with PYTHIA 8.210. The cross-sections were calculated at NLO QCD and NLO EW accuracy using MADGRAPH5_AMC@NLO as reported in ref. [54]. In the case of $t \bar{t} \ell \ell$ the cross-section is additionally scaled by an off-shell correction estimated at one-loop level in $\alpha_{\mathrm{S}}$. For all processes, the A14 set of PYTHIA 8 parameters was used, together with the NNPDF2.3 LO PDF set. EVTGEN v1.2.0 was used to model properties of the $b$ - and $c$-hadron decays. The contribution from $t \bar{t}+H$ was checked and found to be negligible.

Events containing $W$ or $Z$ bosons associated with jets were simulated using the SHERPA v2.2.1 generator. Matrix elements were calculated for up to two partons at NLO and four partons at LO using the Comix [55] and OpenLoops [56] matrix element generators and merged with the SHERPA parton shower [57] using the ME+PS@NLO prescription [58]. The NNPDF3.0 NNLO PDF set [40] was used in association with a tuning performed by the SHERPA authors.

Diboson processes with one hadronically decaying boson accompanied by one charged lepton and one neutrino, two charged leptons or two neutrinos were simulated using Sherpa v2.1.1. The calculations include one additional parton at NLO for $Z Z \rightarrow 2 \ell+q \bar{q}$ and $Z Z \rightarrow 2 \nu+q \bar{q}$ only, and up to three additional partons at LO using the ComIX and OpenLoops matrix element generators and merged with the Sherpa parton shower using the ME+PS@NLO prescription. The NNPDF3.0 NNLO PDF set was used in conjunction with a dedicated parton shower tuning developed by the SHERPA authors. Diboson processes with four charged leptons, three charged leptons and one neutrino, or two charged leptons and two neutrinos, are found to be negligible.

Theoretical uncertainties are considered for all simulated samples. By far the most important process simulated in this analysis is $t \bar{t}$ production, and several samples produced with different configurations, as explained below, are compared to estimate the uncertainty in this background. Samples were produced with the factorisation and renormalisation scales varied coherently up and down by a factor of two, and with parameters set to provide more/less radiation in the parton shower [59]. Additionally, to account for uncertainties from the parton shower modelling and generator choice, the nominal sample is compared to a sample generated with Powheg-Box interfaced to Herwig 7 [60] using the H7UE tune [61] and the MMHT2014 LO PDF set [62], as well as samples generated with MAdGraph5_aMC@NLO interfaced to Pythia 8. These alternative samples each use the NNPDF3.0 NLO PDF for the matrix element calculation. The comparison with samples which vary the amount of additional radiation contributes the largest uncertainty in the $t \bar{t}$ signal region predictions. Similar alternative samples are used to assess the uncertainties in single-top-quark production, whereas uncertainties in other processes are handled via scale variations in the corresponding generator.

Full simulation was used for all background MC samples, ensuring an accurate representation of detector effects. Further details of samples can be found in refs. [59, 63, 64].

A number of SUSY signal model samples were simulated using the ATLAS fast detector simulation [31] to allow the interpretation of the search results in terms of SUSY parameters. Substantial cross-sections are possible for production of gluinos. The resulting 


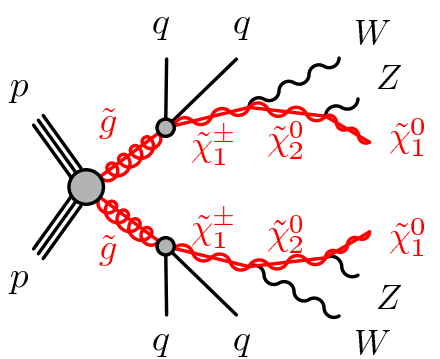

(a) Two-step decay

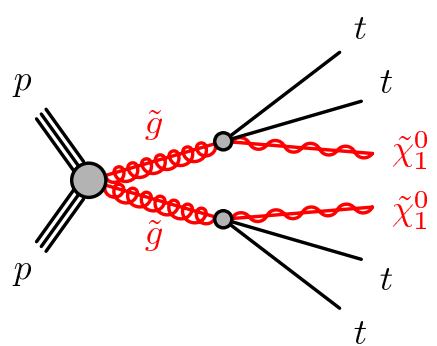

(b) Off-shell top squarks

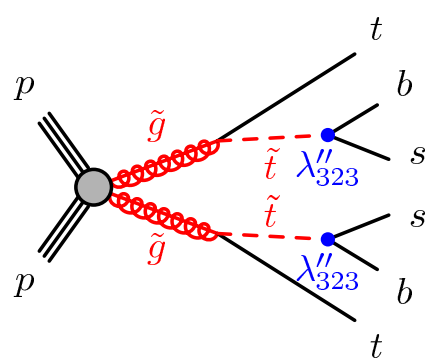

(c) RPV

Figure 1. Pseudo-Feynman diagrams for the different signal models used in this search. In (c), $\lambda_{323}^{\prime \prime}$ is one of the couplings of the third-generation squark to quarks in the RPV model.

cascade decays result in a large multiplicity of jets, and may also exhibit an unusually high heavy-flavour content or atypically large jet masses.

The first type of SUSY signal simulated is a simplified model, in which gluinos are pair-produced and then decay through an off-shell squark via the cascade:

$$
\begin{aligned}
\tilde{g} & \rightarrow q+\bar{q}^{\prime}+\tilde{\chi}_{1}^{ \pm} \quad\left(q, q^{\prime} \in\{u, d, s, c\}\right), \\
\tilde{\chi}_{1}^{ \pm} & \rightarrow W^{ \pm}+\tilde{\chi}_{2}^{0} \\
\tilde{\chi}_{2}^{0} & \rightarrow Z+\tilde{\chi}_{1}^{0}
\end{aligned}
$$

where the quarks are only permitted to be from the first two generations. The parameters of the model are the masses of the gluino, $m_{\tilde{g}}$, and of the lightest neutralino, $m_{\tilde{\chi}_{1}^{0}}$. The mass of the $\tilde{\chi}_{1}^{ \pm}$is constrained to be $\left(m_{\tilde{g}}+m_{\tilde{\chi}_{1}^{0}}\right) / 2$, and the mass of the $\tilde{\chi}_{2}^{0}$ is set to $\left(m_{\tilde{\chi}_{1}^{ \pm}}+m_{\tilde{\chi}_{1}^{0}}\right) / 2$. A diagram of this 'two-step' simplified model is shown in figure 1(a).

An additional signal model to which this analysis has significant sensitivity is gluinomediated top squark $\left(\tilde{t}_{1}\right)$ production, in which top-quark-rich final states are produced as shown in figure 1(b). This model manifests itself as top quark production via either offshell or on-shell top squarks. In the off-shell model, pair-production of gluinos is followed by their decay with a $100 \%$ branching ratio into $t \bar{t}+\tilde{\chi}_{1}^{0}$, through a virtual top squark. Naturalness arguments for SUSY favour light gluinos, top squarks, and Higgsinos, so they motivate consideration of this final state.

Permitting non-zero RPV couplings allows consideration of another variety of gluinomediated top squark production, wherein the last step of the decay proceeds via a baryonnumber-violating interaction: $\tilde{t}_{1} \rightarrow \bar{s}+\bar{b}$ (with charge conjugates implied). Such RPV models may give rise to final states with missing transverse momentum, for example from leptonic decays of $W$ bosons produced in top quark decays. The current analysis accepts final states with sufficiently low missing transverse momentum to be sensitive to these RPV scenarios. Figure 1(c) presents the RPV simplified model considered, for which the coupling strength induces prompt top squark decays.

The signal samples were generated using MADGRAPH5_aMC@NLO interfaced to Pythia 8 with the A14 tune for the modelling of the parton shower, hadronisation and 
underlying event. The versions of the generators used for the two-step and RPV simplified models were MADGraPh5_aMC@NLO v2.6.2 with Pythia 8.212, and for the gluinomediated top squark model MadGraph5_aMC@NLO v2.3.3 with Pythia 8.212. The matrix element calculation was performed at tree level and includes the emission of up to two additional partons. The PDF set used for the generation was NNPDF2.3 LO. The EvTGEN v1.6.0 program was used to simulate properties of the $b$ - and $c$-hadron decays. The matrix-element to parton-shower matching was done using the CKKW-L prescription [65], with a matching scale set to $m_{\tilde{g}} / 4$.

Signal cross-sections were calculated to approximate NNLO in the strong coupling constant, adding the resummation of soft gluon emission at NNLL accuracy [66-73]. The nominal cross-section and its uncertainty were determined using the PDF4LHC15_mc PDF set, following the recommendations of ref. [74].

\section{Reconstruction and particle identification}

Primary vertices are reconstructed using at least two charged-particle tracks with $p_{\mathrm{T}}>$ $500 \mathrm{MeV}$ measured by the ID [75]. The primary vertex with the largest sum of squared track transverse momenta $\left(\sum p_{\mathrm{T}}^{2}\right)$ is designated as the hard-scatter vertex.

Jets are reconstructed using the anti- $k_{t}$ [76-78] jet algorithm with radius parameter $R=0.4$. It uses as inputs particle-flow objects. These are charged-particle tracks matched to the hard-scatter vertex with the requirement $\left|z_{0} \sin \theta\right|<2.0 \mathrm{~mm}$, where $z_{0}$ is the longitudinal impact parameter, and calorimeter energy clusters surviving an energy subtraction algorithm that removes the calorimeter deposits of good-quality tracks originating from any vertex [17]. To eliminate jets containing a large energy contribution from pile-up, jets are tested for compatibility with the hard-scatter vertex with the jet vertex tagger (JVT) discriminant, utilising information from the ID tracks associated with the jet [79]. Any jets with $20 \mathrm{GeV}<p_{\mathrm{T}}<120 \mathrm{GeV}$ and $|\eta|<2.4$ for which JVT $<0.5$ are considered to originate from pile-up and are therefore rejected from the analysis. After the selection above is applied, only jets with $p_{\mathrm{T}}>20 \mathrm{GeV}$ and $|\eta|<2.8$ are considered in this analysis, with the exception of the $E_{\mathrm{T}}^{\text {miss }}$ calculation, for which jets in the range $2.8 \leq|\eta| \leq 4.5$ are also used. Hadronically decaying $\tau$-leptons are not discriminated from other hadronic jets.

Reconstructed $R=0.4$ jets with $p_{\mathrm{T}}>20 \mathrm{GeV}$ and $|\eta|<2.0$ are reclustered to form large-radius jets using the anti- $k_{t}$ algorithm with radius parameter $R=1.0$ [80]. The input jets are required to pass an overlap removal procedure accounting for ambiguities between jets and leptons, as discussed below. Large-radius jets are retained for analysis if they have $p_{\mathrm{T}}>100 \mathrm{GeV}$ and $|\eta|<1.5$.

Jets containing $b$-hadrons and which are within the ID acceptance $(|\eta|<2.5)$ are identified as $b$-tagged jets using a multivariate algorithm that exploits the impact parameters of the charged-particle tracks, the presence of secondary vertices, and the reconstructed flight paths of $b$ - and $c$-hadrons inside the jet [81]. The output of the algorithm is a single discriminant value which signifies the likelihood that the jet contains a $b$-hadron. This analysis considers jets to be $b$-tagged if the discriminant exceeds a threshold that results in an average identification efficiency of $70 \%$ for jets containing $b$-hadrons in simulated $t \bar{t}$ 
events [82]. In the same event sample, a rejection factor of approximately 300 is reached for jets initiated by light quarks and gluons and 8.9 for jets initiated by charm quarks.

Electron candidates are reconstructed from energy deposits in the EM calorimeter that are matched to charged-particle tracks in the ID [83]. 'Baseline electrons' are required to satisfy $p_{\mathrm{T}}>7 \mathrm{GeV}$ and $|\eta|<2.47$. They are identified using the 'loose' operating point provided by a likelihood-based algorithm [83]. Electrons with $p_{\mathrm{T}}>20 \mathrm{GeV}$ are defined as 'signal electrons' if they pass a 'tight' likelihood selection including impact parameter restrictions and the 'GradientLoose' isolation requirement [84] in addition to the 'baseline electron' preselection. To achieve additional rejection of background electrons from nonprompt sources, signal electron tracks must be matched to the hard-scatter vertex with a longitudinal impact parameter $\left|z_{0} \sin \theta\right|<0.5 \mathrm{~mm}$ and a transverse impact parameter significance $\left|d_{0}\right| / \sigma\left(d_{0}\right)<5$. Signal electrons are used in leptonic control regions, as described in section 6.2. The electron reconstruction and identification efficiency in simulated samples are corrected by factors determined by data-MC comparison using a given final state [84].

Photon candidates are identified using 'tight' criteria for lateral shower shapes in the first and second layers of the EM calorimeter, as well as for the degree of hadronic shower leakage [83]. Acceptance requirements of $p_{\mathrm{T}}>40 \mathrm{GeV}$ and $|\eta|<2.37$ are applied. Additionally, photons falling in the region $1.37<|\eta|<1.52$ are removed, to avoid a region of the calorimeter with limited instrumentation.

Muon candidates are reconstructed from matching tracks in the ID and muon spectrometer, refined through a global fit which uses the hits from both subdetectors [85]. 'Baseline muons' must have $p_{\mathrm{T}}>6 \mathrm{GeV}$ and $|\eta|<2.7$, and satisfy the 'medium' identification criteria. Similarly to electrons, the longitudinal impact parameter $z_{0}$ relative to the hard-scatter vertex is required to satisfy $\left|z_{0} \sin \theta\right|<0.5 \mathrm{~mm}$. Muons are characterised as 'signal muons' if they have a higher transverse momentum, $p_{\mathrm{T}}>20 \mathrm{GeV}$, and satisfy the 'GradientLoose' isolation requirement [85], as well as a further transverse impact parameter restriction $\left|d_{0}\right| / \sigma\left(d_{0}\right)<3$. Signal muons are used in leptonic control regions, as described in section 6.2. Muon reconstruction and identification efficiencies in simulated samples are corrected with factors evaluated by a data-MC comparison [86].

To resolve the reconstruction ambiguities between electrons, muons, photons and jets, an overlap removal procedure is applied to baseline objects. First, any electron sharing an ID track with a muon is rejected. If it shares the same ID track with another electron, the one with lower $p_{\mathrm{T}}$ is discarded. Next, photons with $\Delta R<0.4$ relative to an electron or a muon are discarded. Subsequently, non- $b$-tagged jets are rejected if they lie within $\Delta R=0.2$ of an electron or if the jet has no more than three tracks with $p_{\mathrm{T}}>500 \mathrm{MeV}$, or contains an ID track matched to a muon such that $p_{\mathrm{T}}^{\text {jet }}<2 p_{\mathrm{T}}^{\mu}$ and the muon track has more than $70 \%$ of the sum of the transverse momenta of all tracks in the jet, such that the jet resembles radiation from the muon. Finally, electrons or muons with $\Delta R<0.4$ from a surviving jet are eliminated and jets with $\Delta R<0.4$ from photons are removed.

The missing transverse momentum, $E_{\mathrm{T}}^{\text {miss }}$, is defined as the magnitude of the negative vector sum of the transverse momenta of baseline electrons and muons, photons and jets, which pass an overlap removal procedure, based on removing duplicated energy contributions and therefore distinct from that used for jet/lepton disambiguation. A 'soft term' 
is added to recover the contributions from other low- $p_{\mathrm{T}}$ particles $[87,88]$. The soft term is constructed from all tracks that are not associated with any of the preceding objects, but that are associated with the hard-scatter vertex. In this way, the missing transverse momentum is adjusted for the best calibration of the leptons, photons and jets, while maintaining pile-up robustness in the soft term.

The $E_{\mathrm{T}}^{\text {miss }}$ significance $\mathcal{S}\left(E_{\mathrm{T}}^{\text {miss }}\right)$ is defined in order to test the hypothesis that the total transverse momentum carried by non-interacting particles is equal to zero against the hypothesis that it is different from zero. It quantifies the degree to which the measured $E_{\mathrm{T}}^{\text {miss }}$ is inconsistent with originating purely from fluctuations and mismeasurements. A large value indicates that the $E_{\mathrm{T}}^{\text {miss }}$ genuinely originates from non-interacting particles. In this search, the variable $\mathcal{S}\left(E_{\mathrm{T}}^{\mathrm{miss}}\right)$ characterises the $E_{\mathrm{T}}^{\text {miss }}$ according to the $p_{\mathrm{T}}, p_{\mathrm{T}}$ resolution, and $\phi$ resolution of all objects in the event and is defined [18] as:

$$
\mathcal{S}\left(E_{\mathrm{T}}^{\mathrm{miss}}\right)=\frac{E_{\mathrm{T}}^{\mathrm{miss}}}{\sqrt{\sigma_{\mathrm{L}}^{2}\left(1-\rho_{\mathrm{LT}}^{2}\right)}} .
$$

In this definition, $\sigma_{\mathrm{L}}$ is the total expected longitudinal resolution of all objects in the event as a function of the $p_{\mathrm{T}}$ of each object. Likewise, $\rho_{\mathrm{LT}}$ is the correlation factor between all longitudinal and transverse object resolutions. The resolution of the soft term is fixed to $8.9 \mathrm{GeV}$ in each of the transverse coordinates. The resolution of each jet is further modified by the probability that the jet is a pile-up jet mistakenly tagged as being from the hard-scatter interaction, parameterised in $p_{\mathrm{T}}, \eta$ and JVT. By individually accounting for these resolutions, this 'object-based' $\mathcal{S}\left(E_{\mathrm{T}}^{\mathrm{miss}}\right)$ definition captures the response of the ATLAS detector and reconstruction algorithms better than the simpler $\mathcal{S}\left(E_{\mathrm{T}}^{\text {miss }}\right)$ proxies used in previous searches (such as in ref. [16]).

\section{Event selection}

Signal events for this analysis are characterised by a large jet multiplicity, beyond that generated by high cross-section $\mathrm{SM}$ processes, combined with an $E_{\mathrm{T}}^{\text {miss }}$ value that is significantly larger than that expected purely from detector resolution and mismeasurement effects. Several signal regions are defined that select a minimum jet multiplicity and further require a large value of $\mathcal{S}\left(E_{\mathrm{T}}^{\text {miss }}\right)$.

The common selection of events for the signal regions is as follows. Events are rejected if no primary vertex is reconstructed. To limit the contribution from SM background processes in which neutrinos are produced via the decay $W \rightarrow \ell \nu$, leading to significant $E_{\mathrm{T}}^{\text {miss }}$, events containing any baseline electron or muon with $p_{\mathrm{T}}>10 \mathrm{GeV}$ surviving the overlap removal are rejected.

Biases in the $E_{\mathrm{T}}^{\text {miss }}$ due to pile-up jets surviving the JVT selection are removed by excluding events for which a jet $j$ with $60 \mathrm{GeV}<p_{\mathrm{T}}<70 \mathrm{GeV}$ and JVT $<0.2$ lies opposite to the $E_{\mathrm{T}}^{\text {miss }}$ vector in $\phi\left(\left|\Delta \phi\left(j, E_{\mathrm{T}}^{\text {miss }}\right)\right|>2.2\right)$. Likewise, events are rejected if they contain a jet with $p_{\mathrm{T}}>50 \mathrm{GeV}$ and $|\eta|<2.0$ pointing in $\eta-\phi$ towards a region in which tile calorimeter modules were disabled. 
Subsequently, restrictions on the jet multiplicity $N_{\text {jet }}$ are imposed; only jets with $p_{\mathrm{T}}>$ 50 (80) $\mathrm{GeV}$ and $|\eta|<2.0$ are considered as signal jets and therefore used in the $N_{\text {jet }}$ selection. These selections are abbreviated as $j 50$ ( $j 80)$, for which the corresponding jet multiplicities are denoted $N_{\text {jet }}^{50}\left(N_{\text {jet }}^{80}\right)$. The lower and higher jet- $p_{\mathrm{T}}$ thresholds provide sensitivity to a variety of potential SUSY mass spectra and ensure that the corresponding trigger selections give efficiencies in simulation and in data that match to within $2 \%$.

A requirement of $\mathcal{S}\left(E_{\mathrm{T}}^{\mathrm{miss}}\right)>5$ is the last element of the common selection. This criterion eliminates the vast majority of SM multijet and other background events with low $E_{\mathrm{T}}^{\text {miss }}$, while retaining sensitivity to a broad range of potential signals.

Motivated by the likelihood of heavy-flavour jets being produced from cascade decays, events are classified by their $b$-jet multiplicity. Events passing the common selection may then be categorised according to three classes depending on whether they satisfy $N_{b \text {-jet }}=0$, $N_{b \text {-jet }}=1$ or $N_{b \text {-jet }} \geq 2$.

Should SUSY particles be produced and decay through a long decay chain, or provide enough kinetic energy to significantly boost heavy particles such as top quarks or SM electroweak bosons, signal events might be characterised not only by an unusually large jet multiplicity but also by the formation of large-radius jets with high masses. The kinematic structure of SM events, by contrast, does not produce a high rate of events containing large-radius jets with a mass greater than the top quark mass. On this basis, the selection variable $M_{\mathrm{J}}^{\Sigma}$ is defined to be the sum of the masses $m_{j}^{R=1.0}$ of the large-radius jets

$$
M_{\mathrm{J}}^{\Sigma}=\sum_{j} m_{j}^{R=1.0}
$$

where the sum is over the large-radius jets that satisfy $p_{\mathrm{T}_{j}^{R}}^{R=1.0}>100 \mathrm{GeV}$ and $\left|\eta_{j}^{R=1.0}\right|<$ 1.5 , as described in section 4 . After the common selection and $N_{b \text {-jet }}$ classification, selected events may be further partitioned into three $M_{\mathrm{J}}^{\Sigma}$ bins:

- $M_{\mathrm{J}}^{\Sigma} \leq 340 \mathrm{GeV}$;

- $340 \mathrm{GeV}<M_{\mathrm{J}}^{\Sigma} \leq 500 \mathrm{GeV}$;

- $M_{\mathrm{J}}^{\Sigma}>500 \mathrm{GeV}$.

As the bin boundaries are at approximately twice and three times the top quark mass, the residual irreducible backgrounds remaining in the higher $M_{\mathrm{J}}^{\Sigma}$ bins are respectively top quark pair production in association with vector bosons and processes with four top quarks, both of which have a very small rate.

The subselections in $N_{b \text {-jet }}$ and $M_{\mathrm{J}}^{\Sigma}$ are used in two subanalyses. First, a set of multibin signal selections is defined to improve the exclusion reach in the absence of an observed excess. Starting from the common selections, shown in table 1 , for $N_{\text {jet }}^{50} \geq 8$, the selected events are partitioned by $N_{b \text {-jet }}$ and $M_{\mathrm{J}}^{\Sigma}$ according to the thresholds above, defining nine statistically independent bins, as shown in table 2. The same subdivision is applied for events satisfying the $N_{\text {jet }}^{50} \geq 9$ and $N_{\text {jet }}^{50} \geq 10$ common selections. The statistical analyses of the three different $N_{\text {jet }}^{50}$ selections are performed separately since the selections are not 


\begin{tabular}{|c|cr|}
\hline Selection criterion & \multicolumn{2}{|c|}{ Selection ranges } \\
\hline Jet multiplicity, $N_{\text {jet }}$ & $N_{\text {jet }}^{50} \geq\{8,9,10,11,12\}$ & $N_{\text {jet }}^{80} \geq 9$ \\
\hline \multirow{2}{*}{ Trigger thresholds } & $2015-2016: 6$ jets, $E_{\mathrm{T}}>45 \mathrm{GeV}$ & $2015: 5$ jets, $E_{\mathrm{T}}>70 \mathrm{GeV}$ \\
& $2016: 5$ jets, $E_{\mathrm{T}}>65 \mathrm{GeV}$ \\
& $2017-2018: 7$ jets, $E_{\mathrm{T}}>45 \mathrm{GeV} \quad 2017-2018: 5$ jets, $E_{\mathrm{T}}>70 \mathrm{GeV}$ \\
\hline Lepton veto & 0 baseline leptons, $p_{\mathrm{T}}>10 \mathrm{GeV}$ \\
\hline$E_{\mathrm{T}}^{\text {miss }}$ significance, $\mathcal{S}\left(E_{\mathrm{T}}^{\text {miss }}\right)$ & \multicolumn{2}{|c|}{$\mathcal{S}\left(E_{\mathrm{T}}^{\text {miss }}\right)>5.0$} \\
\hline
\end{tabular}

Table 1. Summary of common selections prior to the categorisations based on $N_{b \text {-jet }}$ and $M_{\mathrm{J}}^{\Sigma}$.

\begin{tabular}{|l|r|c|c|}
\hline Signal region & $N_{\text {jet }}^{50}$ & $N_{b \text {-jet }}$ & $M_{\mathrm{J}}^{\Sigma}[\mathrm{GeV}]$ \\
\hline SR-8ij50 multi-bin & $\geq 8$ & & \\
\cline { 1 - 1 } SR-9ij50 multi-bin & $\geq 9$ & $=0,=1, \geq 2$ & $(0,340],(340,500],(500, \infty)$ \\
\hline SR-10ij50 multi-bin & $\geq 10$ & & \\
\hline
\end{tabular}

Table 2. Signal region subdivisions for the multi-bin fit in the $\{8,9,10\} \mathrm{ij} 50$ analysis channels. The selections on these variables apply to the signal regions as well as to the multijet template and validation regions described later in section 6 . For each inclusive jet-multiplicity fit, nine regions are generated combining the $N_{b \text {-jet }}$ and $M_{\mathrm{J}}^{\Sigma}$ selections.

statistically independent. For example, one of the nine bins in the 8-jet selection (e.g. $\left.N_{b \text {-jet }}=0, M_{\mathrm{J}}^{\Sigma}>500\right)$ includes all events in the corresponding bin for the 9- and 10-jet selections. The inputs to the statistical analysis also include validation regions (VRs) and control regions (CRs), further discussed in section 6 .

Additionally, an ensemble of single-bin regions is defined making use of inclusive selections in $N_{b \text {-jet }}$ and $M_{\mathrm{J}}^{\Sigma}$, which are designed to reject the SM hypothesis with high confidence, should new physics be present. Following an optimisation procedure to maximise coverage over the benchmark signal models, the signal regions defined in table 3 were considered. To give an indication of their relevance, the two-step gluino cascade decay motivates the SR-10ij50-0ib-MJ340 and SR-10ij50-0ib-MJ500 regions while the SR-9ij50-0ib-MJ340 and SR-10ij50-1ib-MJ500 regions are optimised for sensitivity to gluino decays into $t \bar{t}+\tilde{\chi}_{1}^{0}$. The SR-10ij50-1ib-MJ500 selection also provides the best sensitivity to RPV gluino decays.

If an excess of data is observed over the background predictions, its significance can be quantified in the single-bin regions. If on the contrary the data are consistent with the background-only hypothesis, signal models are excluded using the constraint achieved by whichever signal region is expected to be most sensitive to the model, whether it is a singleor multi-bin selection.

\section{Background estimation}

The signal selection in this analysis demands higher jet multiplicities than can practically be generated with fixed-order multi-leg matrix element MC generators for most SM 


\begin{tabular}{|l|rrrc|}
\hline Signal region & $N_{\text {jet }}^{50}$ & $N_{\text {jet }}^{80}$ & $N_{b \text {-jet }}$ & $M_{\mathrm{J}}^{\Sigma}[\mathrm{GeV}]$ \\
\hline SR-8ij50-0ib-MJ500 & $\geq 8$ & - & - & $\geq 500$ \\
SR-9ij50-0ib-MJ340 & $\geq 9$ & - & - & $\geq 340$ \\
SR-10ij50-0ib-MJ340 & $\geq 10$ & - & - & $\geq 340$ \\
SR-10ij50-0ib-MJ500 & $\geq 10$ & - & - & $\geq 500$ \\
SR-10ij50-1ib-MJ500 & $\geq 10$ & - & $\geq 1$ & $\geq 500$ \\
SR-11ij50 & $\geq 11$ & - & - & - \\
SR-12ij50-2ib & $\geq 12$ & - & $\geq 2$ & - \\
SR-9ij80 & - & $\geq 9$ & - & - \\
\hline
\end{tabular}

Table 3. Summary of signal region criteria for single-bin selections. The requirements in multiplicity and $M_{\mathrm{J}}^{\Sigma}$ are inclusive in all cases. A dash ('-') indicates that no requirement is applied to the corresponding variable. The requirement $\mathcal{S}\left(E_{\mathrm{T}}^{\mathrm{miss}}\right)>5$ is applied to all bins.

background processes. The kinematic requirements also challenge the parton shower approximations that are used to obtain higher multiplicities than are possible in fixed-order calculations. For this reason the analysis was designed in a manner that allows the expected yields in the signal regions for each of the most important backgrounds to be determined either from fully data-driven methods or by using measurements of ATLAS data to calibrate and to confirm the predictions of the MC simulations.

Three different broad classes of backgrounds are identified, and a different strategy is employed for each.

The first class of background is multijet production through QCD processes in which detector resolution effects produce an apparent momentum imbalance even in the absence of any true $E_{\mathrm{T}}^{\mathrm{miss}}$. This class includes any contribution to the $E_{\mathrm{T}}^{\text {miss }}$ from neutrinos when semileptonic decays of $b$ - and $c$-hadrons are present in those jets. The probability distribution of jet mismeasurement from either source can be measured with high statistical precision in data events with lower jet multiplicity. The distribution of $\mathcal{S}\left(E_{\mathrm{T}}^{\mathrm{miss}}\right)$ is observed to be almost independent of the jet multiplicity, and so the expected yield in each signal region can be determined using a data-driven background template of $\mathcal{S}\left(E_{\mathrm{T}}^{\mathrm{miss}}\right)$ extracted from lower jet-multiplicity data events. Section 6.1 describes this method fully.

The second class of backgrounds consists of processes which contain true momentum imbalance caused by the production of neutrinos in association with charged leptons (excluding semileptonic $b$ - and $c$-hadron decays, which fall in the first class). The dominant backgrounds in this second class are from $t \bar{t}$ and $W+$ jets production. For such backgrounds, auxiliary measurements of the data in control regions (CRs) are used to normalise the predictions from MC simulation. This control region approach is described in detail in section 6.2 .

The third class comprises subdominant backgrounds such as events from leptonically decaying $Z$ bosons and associated jets $(Z+$ jets), single-top-quark production, diboson production of $W W, W Z$, or $Z Z$, and the production of $t \bar{t}$ in association with heavier particles 


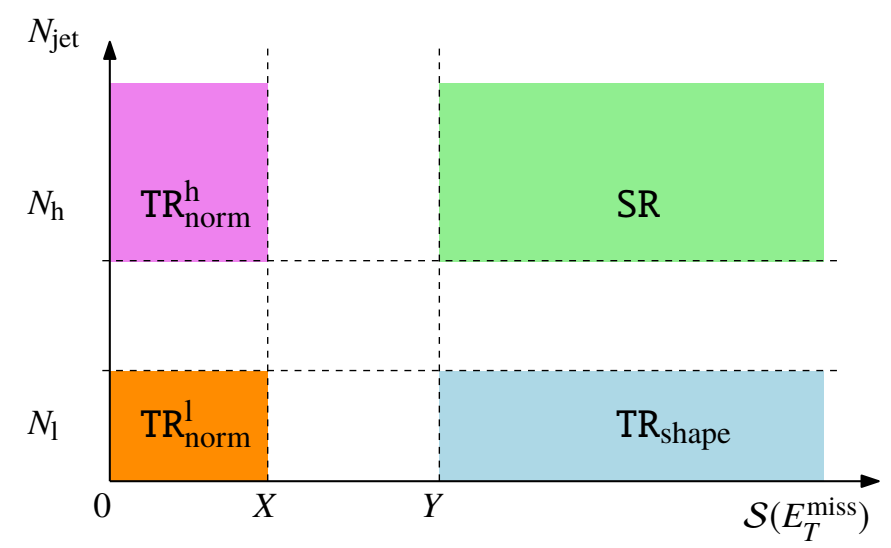

Figure 2. Schematic of the kinematic regions used in the multijet template estimate for the multijet background. SR represents any signal region, while the three TR variations are regions used to extract and normalise the corresponding background prediction.

- this final item covers the production of three or four top quarks, $t \bar{t}+W, t \bar{t}+Z$, and $t \bar{t}+W W$ production, and is collectively denoted by ' $t \bar{t}+X$ '. The expected yields from these backgrounds are found to be sufficiently small that they may be estimated with adequate precision from MC simulation alone.

The final determination of the yield from each of the first two classes of backgrounds is obtained using a simultaneous fit including control regions, as described in section 6.3.

\subsection{Multijet background}

The analysis is designed such that a data-driven method can be used for the estimation of the dominant multijet background. This technique relies on the approximate invariance of the multijet $\mathcal{S}\left(E_{\mathrm{T}}^{\mathrm{miss}}\right)$ distribution with respect to changes in the jet multiplicity. The procedure is also referred to as the multijet template estimate and is schematically shown in figure 2, which illustrates various template regions (TRs) used to extract the prediction.

The predicted multijet background yield in a region $\hat{N}\left[a<\mathcal{S}\left(E_{\mathrm{T}}^{\mathrm{miss}}\right)<b\right]$ with high jet multiplicity $\left(N_{\mathrm{h}}\right)$ and $\mathcal{S}\left(E_{\mathrm{T}}^{\text {miss }}\right)$ in the range $(a, b)$ is obtained from the measured yield $N_{\mathrm{TR}_{\text {shape }}}$ in a lower jet-multiplicity $\left(N_{\mathrm{l}}\right)$ template region $\mathrm{TR}_{\text {shape }}$ through the relation

$$
\hat{N}\left[a<\mathcal{S}\left(E_{\mathrm{T}}^{\mathrm{miss}}\right)<b\right]=\frac{N_{\mathrm{TR}_{\mathrm{norm}}^{\mathrm{h}}}}{N_{\mathrm{TR}_{\mathrm{norm}}^{1}}} N_{\mathrm{TR}_{\text {shape }}}\left[a<\mathcal{S}\left(E_{\mathrm{T}}^{\mathrm{miss}}\right)<b\right] .
$$

In this equation, the regions for template normalisation $\mathrm{TR}_{\text {norm }}^{l}$ and $\mathrm{TR}_{\text {norm }}^{\mathrm{h}}$, at lower and higher jet multiplicity respectively, require $\mathcal{S}\left(E_{\mathrm{T}}^{\mathrm{miss}}\right)<2$ in order to reduce signal and leptonic background contamination. The fraction of signal contamination for non-excluded parameter values in the target models is found to range from $<0.1 \%$ to $10 \%$ depending on the region.

The multijet yields in the template regions $T R_{\text {shape }}, \mathrm{TR}_{\text {norm }}^{1}$ and $\mathrm{TR}$ norm are extracted by subtracting the predicted contributions from the two leptonic background classes from the yield in data. For signal regions requiring $N_{\text {jet }}^{50} \geq 8-12$, the $\mathrm{TR}_{\text {norm }}^{1}$ and $\mathrm{TR}_{\text {shape }}$ are defined using events with $N_{\text {jet }}^{50}=7$. The criterion $N_{\text {jet }}^{80}=5$ defines the corresponding template 


\begin{tabular}{|l|ccccc|}
\hline $\mathcal{S}\left(E_{\mathrm{T}}^{\text {miss }}\right)$ range & $0-2$ & $2-3$ & $3-4$ & $4-5$ & $>5$ \\
\hline$N_{\text {jet }}^{80}=5$ & $\mathrm{TR}_{\text {norm }}^{1}$ & - & - & - & $\mathrm{TR}_{\text {shape }}$ \\
$N_{\text {jet }}^{80}=6$ & - & - & - & - & $\mathrm{VR}_{N_{\text {jet }}}$ \\
$N_{\text {jet }}^{80} \geq 9$ & $\mathrm{TR}_{\text {norm }}^{\mathrm{h}}$ & - & $\mathrm{QCR}$ & $\mathrm{VR}_{\mathcal{S}\left(E_{\mathrm{T}}^{\text {miss }}\right)}$ & $\mathrm{SR}$ \\
\hline
\end{tabular}

Table 4. Illustration of the main multijet template, control and validation regions in $N_{\text {jet }}$ and $\mathcal{S}\left(E_{\mathrm{T}}^{\mathrm{miss}}\right)$ corresponding to the $\mathrm{SR}-9 \mathrm{ij} 80$ signal region. The template regions used to derive predictions in the control and validation regions are not shown.

regions for the signal region selecting events with $N_{\text {jet }}^{80} \geq 9$. The $\mathrm{TR}_{\text {norm }}^{\mathrm{h}}$ selection requires the same jet multiplicity as the corresponding signal region.

A residual dependence of the multijet template estimate on event kinematics was observed previously [15]. This secondary dependence is addressed by applying the template method differentially in bins of the total scalar sum of jet transverse momenta $H_{\mathrm{T}}=\sum_{j \in \text { jets }} p_{\mathrm{T} j}$, where $p_{\mathrm{T} j}>40 \mathrm{GeV}$. The $H_{\mathrm{T}}$ bin ranges are chosen dynamically to ensure good statistical precision in each bin. The effect of a different choice of the binning on the background estimate is accounted for by a dedicated systematic uncertainty. For each signal region bin, the template estimate is extracted after applying the same $M_{\mathrm{J}}^{\Sigma}$ and $N_{b \text {-jet }}$ selections to all relevant template regions. Residual inaccuracies due to the $N_{\text {jet }}$ extrapolation and potential mismodelling of the simulated backgrounds in the template regions are corrected in a control region (QCR) defined in the range $3<\mathcal{S}\left(E_{\mathrm{T}}^{\mathrm{miss}}\right)<4$, and at the same jet multiplicity as the signal region. The yield in each QCR is used as input to the simultaneous fit described in section 6.3.

To verify the accuracy of the multijet background prediction, two sets of validation regions are defined. These correspond to the two dimensions in which the background prediction is extrapolated:

- $\mathrm{VR}_{N_{\text {jet }}}$ is defined at a jet multiplicity below that of the signal region, and tests the extrapolation in $\mathcal{S}\left(E_{\mathrm{T}}^{\mathrm{miss}}\right)$;

- $\mathrm{VR}_{\mathcal{S}\left(E_{\mathrm{T}}^{\mathrm{miss}}\right)}$ instead is defined in an intermediate $\mathcal{S}\left(E_{\mathrm{T}}^{\mathrm{miss}}\right)$ range between the QCR and the signal region, specifically $4<\mathcal{S}\left(E_{\mathrm{T}}^{\mathrm{miss}}\right)<5$, and tests the extrapolation in $N_{\text {jet }}$.

For the SR-9ij80 signal region, the $\mathrm{VR}_{N_{\text {jet }}}$ requires $N_{\text {jet }}^{80}=6$, a multiplicity between the template regions and the signal region selection. An intermediate jet-multiplicity region is not available for the $j 50$ selections, as the $S R-8 i j 50$ regions are adjacent to the template regions. Instead, the $\mathrm{VR}_{N_{\text {jet }}}$ requires $N_{\text {jet }}^{50}=7$, and the test is performed on a template extracted from data with $N_{\text {jet }}^{50}=6$, selected with a prescaled trigger. The template region $\mathcal{S}\left(E_{\mathrm{T}}^{\text {miss }}\right)$ and $N_{\text {jet }}$ requirements are summarised together with the QCR and VR requirements in table 4 and table 5. Example distributions of $\mathcal{S}\left(E_{\mathrm{T}}^{\mathrm{miss}}\right)$ in the lower jet-multiplicity VRs are shown in figure 3 . 


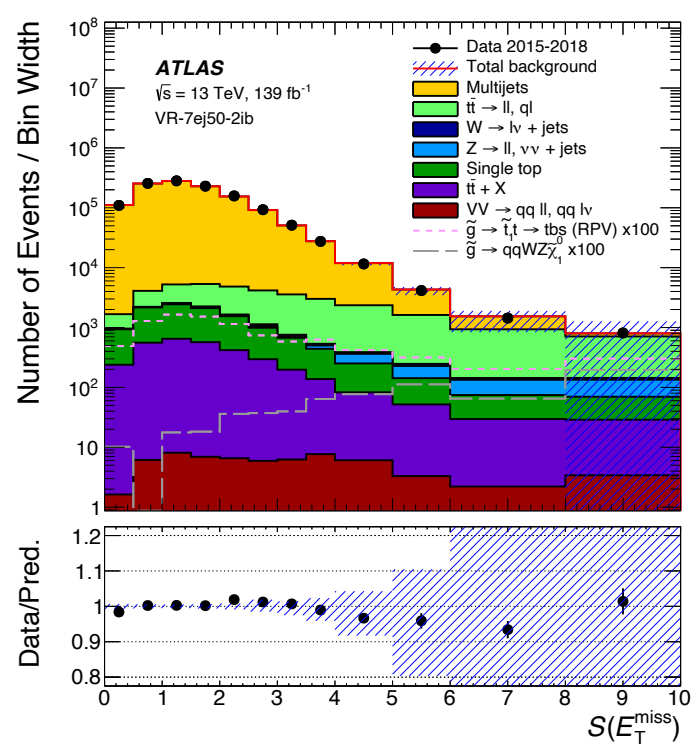

(a) $N_{\text {jet }}^{50}=7, N_{b-\text { jet }} \geq 2$

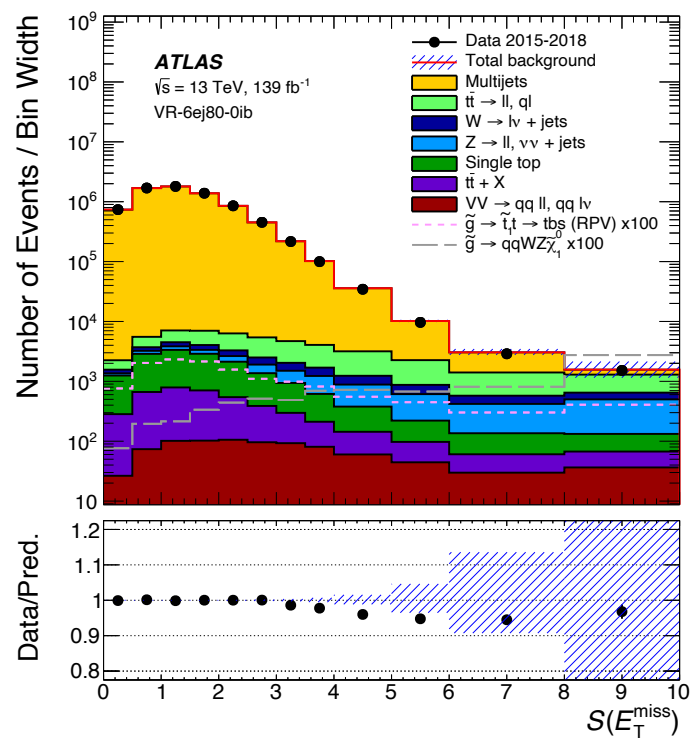

(b) $N_{\text {jet }}^{80}=6, N_{b-\text { jet }} \geq 0$

Figure 3. Distributions of $\mathcal{S}\left(E_{\mathrm{T}}^{\mathrm{miss}}\right)$ for events in two of the validation regions. The upper panel shows the absolute yields for data (black points) and all background subcomponents (histograms), with the combination of statistical and systematic uncertainties shown by the hatched areas. The yields for two benchmark signal models are overlaid, representing $1.6 \mathrm{TeV}$ gluinos decaying into $W$ and $Z$ bosons and a $100 \mathrm{GeV}$ neutralino via intermediate gauginos (long dashed histogram) or instead into $t b s / t b d$ via a $600 \mathrm{GeV}$ top squark through an $R$-parity-violating (RPV) coupling (short dashed histogram). Signal yields are scaled by a factor of 100 for visibility. The lower panel shows the ratio of the data yields to the total SM prediction. 


\begin{tabular}{|l|ccccc|}
\hline $\mathcal{S}\left(E_{\mathrm{T}}^{\text {miss }}\right)$ range & $0-2$ & $2-3$ & $3-4$ & $4-5$ & $>5$ \\
\hline$N_{\text {jet }}^{50}=6$, prescaled data & $\mathrm{TR}_{\text {norm }}^{1, \text { prescale }}$ & - & - & - & $\mathrm{TR}_{\text {shape }}^{\text {prescale }}$ \\
$N_{\text {jet }}^{50}=7$, full dataset & $\mathrm{TR}_{\text {norm }}^{\text {h,prescale }}$ & - & - & - & $\mathrm{VR}_{N_{\text {jet }}}$ \\
\hline$N_{\text {jet }}^{50}=7$, full dataset & $\mathrm{TR}_{\text {norm }}^{1}$ & - & - & - & $\mathrm{TR}_{\text {shape }}$ \\
$N_{\text {jet }}^{50} \geq 8$, full dataset & $\mathrm{TR}_{\text {norm }}^{\mathrm{h}}$ & - & $\mathrm{QCR}$ & $\mathrm{VR}_{\mathcal{S}\left(E_{\mathrm{T}}^{\text {miss }}\right)}$ & $\mathrm{SR}$ \\
\hline
\end{tabular}

Table 5. Illustration of the main multijet template, control and validation regions in $N_{\text {jet }}$ and $\mathcal{S}\left(E_{\mathrm{T}}^{\mathrm{miss}}\right)$ corresponding to the $\mathrm{j} 50$ signal regions. The template regions needed to derive predictions in the control and $\mathrm{VR}_{\mathcal{S}\left(E_{\mathrm{T}}^{\mathrm{miss}}\right)}$ regions are not shown. For the row labelled 'prescaled data', a six-jet trigger was used that collected only a fraction of the Run 2 data. The superscript 'prescale' is used to indicate the template regions used to predict the $\mathrm{VR}_{N_{\text {jet }}}$ background using prescaled data.

\subsection{Leptonic backgrounds}

The SM processes which produce multijet events with one or more leptons are categorised as leptonic backgrounds. Although events containing charged leptons $(e$ or $\mu)$ are excluded from the signal regions, it is still possible for leptonic backgrounds to contaminate them. The veto only applies to events containing electrons or muons, and hence hadronically decaying $\tau$-leptons (originating from top quark or $W$ boson decays) remain a source of background. Such $\tau$-leptons are treated as jets within this analysis, so they may contribute to the jet count if they have sufficient $p_{\mathrm{T}}$, and the momentum lost through any associated neutrinos can also cause these events to enter the signal regions. Additionally, there are cases where the electrons or muons may fall outside of the detector acceptance, or be misreconstructed in the detector, increasing the $E_{\mathrm{T}}^{\mathrm{miss}}$ of the event.

The two largest leptonic backgrounds are from leptonically and semileptonically decaying $t \bar{t}$, and leptonically decaying $W$ bosons produced in association with jets. The estimation of these backgrounds employs the MC simulations described in section 3. To reduce normalisation and modelling uncertainties the background predictions are normalised to data using CRs.

The control regions are designed to be kinematically similar to signal regions, but not to overlap with them. They are designed to enhance the contributions from particular backgrounds, in order to measure those backgrounds cleanly, while being comparatively sparse in signal contamination. Statistical orthogonality between the signal regions and the leptonic CRs is achieved by requiring exactly one electron or muon in CR events. To reduce statistical uncertainties, each $N_{\text {jet }} \geq m$ signal region has a corresponding $\left(N_{\text {jet }} \geq\right.$ $m-1)$ control region, except in the case of signal regions with an $N_{\text {jet }}^{50} \geq 8$ requirement, where there are sufficiently large yields in the control region to match the signal region $N_{\text {jet }} \geq m$ requirement. In order to increase the statistical precision in control regions with higher jet-multiplicity requirements, the $\mathcal{S}\left(E_{\mathrm{T}}^{\mathrm{miss}}\right)$ threshold is reduced to 4 in the leptonic control regions.

Two leptonic control regions are defined for each signal region: the first (WCR) includes a $b$-jet veto to enhance the contribution of $W+$ jets backgrounds, and the second (TCR) requires at least one $b$-jet in the event, enriching the region with the $t \bar{t}$ background. The 


\begin{tabular}{|l|c|}
\hline Selection criterion & Selection ranges \\
\hline Lepton multiplicity & Exactly one signal $e$ or $\mu$ remaining after overlap removal \\
Lepton $p_{\mathrm{T}}$ & $>20 \mathrm{GeV}$ \\
$m_{\mathrm{T}}$ & $<120 \mathrm{GeV}$ \\
Trigger & Same as signal regions \\
Jet $p_{\mathrm{T}},|\eta|$ & Same as signal regions \\
$N_{\text {jet }}$ (including lepton) & $\geq 8$, for $N_{\text {jet }}^{50} \geq 8$ signal regions; \\
$N_{b \text {-jet }}$ & $\geq\left(N_{\text {jet }}^{\mathrm{SR}}-1\right)$, otherwise \\
$M_{\mathrm{J}}^{\Sigma}$ & $=0($ WCR $), \geq 1(\mathrm{TCR})$ \\
$\mathcal{S}\left(E_{\mathrm{T}}^{\text {miss }}\right)$ & Same as signal regions \\
\hline
\end{tabular}

Table 6. Summary of the selections used to define the leptonic control regions.

\begin{tabular}{|c|cc|}
\hline $\begin{array}{c}8,9,10 \mathrm{ij} 50 \\
\text { CR definitions }\end{array}$ & $N_{b \text {-jet }}=0$ & $N_{b \text {-jet }} \geq 1$ \\
\hline$M_{\mathrm{J}}^{\Sigma} \leq 340 \mathrm{GeV}$ & WCR 1 & TCR 1 \\
$340 \mathrm{GeV}<M_{\mathrm{J}}^{\Sigma} \leq 500 \mathrm{GeV}$ & WCR2 & TCR2 \\
$M_{\mathrm{J}}^{\Sigma}>500 \mathrm{GeV}$ & WCR3 & TCR3 \\
\hline
\end{tabular}

Table 7. Control region subdivisions for the background fits for multi-bin signal selections.

signal-region $p_{\mathrm{T}}$ thresholds imposed on the jets also apply to the corresponding control regions. To simulate the effect of $\tau$-leptons being reconstructed as jets in the signal regions, electrons and muons are also included as jets, for the purpose of the corresponding CR selection, provided that they pass the same $p_{\mathrm{T}}$ and $|\eta|$ requirements as the jets in the event. Finally, an upper bound on the transverse mass ${ }^{2}$ computed with the lepton and $E_{\mathrm{T}}^{\text {miss }}$ is applied at $120 \mathrm{GeV}$ in order to reduce contributions from signal processes. This variable has a kinematic endpoint at the $W$ boson mass for leptonically decaying on-shell $W$ bosons, but has no such bound when the lepton and $E_{\mathrm{T}}^{\text {miss }}$ originate from different decays. The control region definitions are summarised in table 6 .

Additional requirements are placed on $M_{\mathrm{J}}^{\Sigma}$ for the control regions in the same manner as for the corresponding signal region selections. In the case of the single-bin selections (table 3), the same threshold is applied. For multi-bin selections, the control regions have three bins, corresponding to the same $M_{\mathrm{J}}^{\Sigma}$ thresholds, as shown in table 7 .

The WCR and TCR are used as inputs to a fit that applies normalisation corrections to the $t \bar{t}$ and $W+$ jets background components, as described in section 6.3.

\subsection{Background normalisation corrections}

Background estimates in the signal region are made more accurate by employing a background likelihood fit based on the control regions, using the methods described in ref. [89]. By means of this fit, the raw estimated yields for the major background components, in-

\footnotetext{
${ }^{2}$ The transverse mass is defined as $\left.m_{\mathrm{T}}=\sqrt{2 p_{\mathrm{T}}^{\ell} E_{\mathrm{T}}^{\mathrm{miss}}\left[1-\cos \left(\Delta \phi\left({\overrightarrow{p_{\mathrm{T}}}}^{\ell}, \vec{E}_{\mathrm{T}}^{\mathrm{miss}}\right)\right)\right.}\right]$, where $p_{\mathrm{T}}^{\ell}$ is the lepton $p_{\mathrm{T}}$.
} 
cluding the multijet, $t \bar{t}$, and $W+$ jets processes, are corrected for mismodelling. For the other background processes, which contribute of the order of $1 \%$ of the SR yields, the nominal MC predictions are used directly. The extent to which the background prediction is compatible with the signal region observation is quantified in the form of a $p$-value $\mathrm{CL}_{\mathrm{b}}$, which is the probability of an upward fluctuation of the event yield relative to the signal region prediction no larger than that observed in data, given the background model. The multijet background, while estimated using a data-driven procedure, is incorporated in the simultaneous fit due to the dependence of the template prediction on the subtraction of other backgrounds which include the simulated $t \bar{t}$ and $W+$ jets estimates. Besides correcting for any residual mismodelling, fitting the multijet component handles the correlations between the systematic uncertainties of the different background components consistently.

In the case of the single-bin regions (table 3), the normalisations of the background components are allowed to vary within their nominal uncertainties, described in section 6.4. For the multi-bin analysis channels (table 2), the additional information available to the fit permits a reduction in the uncertainties, as well as a modification of the event yields to better accommodate the control region measurements. To avoid artificially constraining systematic uncertainties in the background fit due to the high statistical precision, the multijet normalisation region QCR is limited to a single bin with $N_{b \text {-jet }} \geq 0$ and $M_{\mathrm{J}}^{\Sigma} \geq 0$.

For illustration, the pre-fit yields for the SR-8ij50 leptonic CRs are shown in figure 4, demonstrating the extent of the observed mismodelling in the $W+$ jets and $t \bar{t}$ normalisation. The fitted normalisation factors are summarised for all signal selections in figure 5 , and are found to be consistent across the wide range of jet multiplicities probed.

Figure 6 shows the background modelling in the validation regions $\mathrm{VR}_{N_{\text {jet }}}$ and $\mathrm{VR}_{\mathcal{S}\left(E_{\mathrm{T}}^{\text {miss }}\right)}$ for the SR-8ij50 multi-bin analysis. Considering uncertainties, the data yields are in agreement with the predictions after applying the background normalisation factors. Similar levels of agreement are found for the single-bin signal regions, as can be seen in figure 7 . In the $\mathrm{VR}_{\mathcal{S}\left(E_{\mathrm{T}}^{\mathrm{miss}}\right)}$, there is a tendency for the background predictions to mildly overshoot the data, at the level of $10 \%$. This is due to residual kinematic correlations causing the $\mathcal{S}\left(E_{\mathrm{T}}^{\mathrm{miss}}\right)$ distribution not to be entirely independent of the jet multiplicity. Applying flavour-tagging and jet mass selections alters these correlations. In the validation and signal regions, an uncertainty based on the largest observed non-closure at lower jet multiplicities or smaller $\mathcal{S}\left(E_{\mathrm{T}}^{\mathrm{miss}}\right)$ values is applied, and is found to cover the observed discrepancies.

\subsection{Systematic uncertainties}

Systematic uncertainties from the following sources are assessed for the predicted background yields. Experimental systematic uncertainties chiefly include uncertainties in the energy or momentum scales of reconstructed jets and leptons or the missing transverse momentum, as well as the uncertainty in the total integrated luminosity and the magnitude of pile-up corrections. Theoretical uncertainties are assessed by varying the scales (renormalisation, factorisation, resummation, shower matching) at which cross-sections are calculated or by comparison of an ensemble of matrix element and parton shower programs used to generate the predictions. Each source of theoretical uncertainty is correlated across 


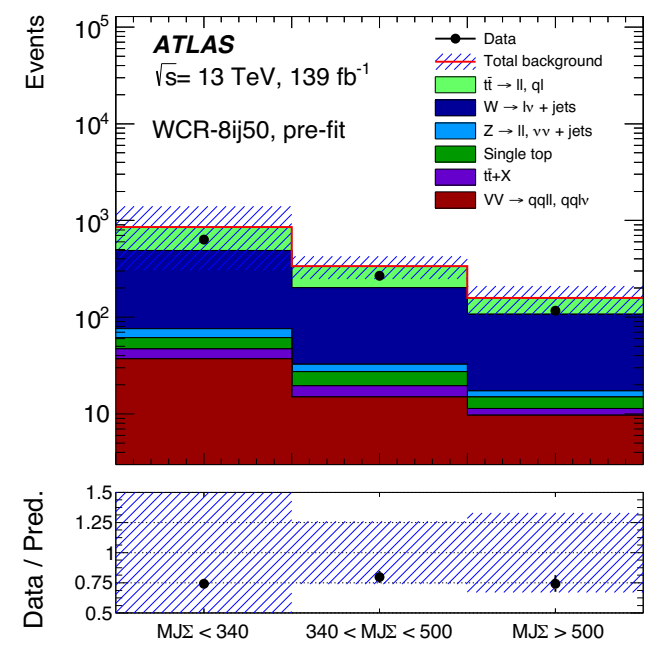

(a) WCR, pre-fit

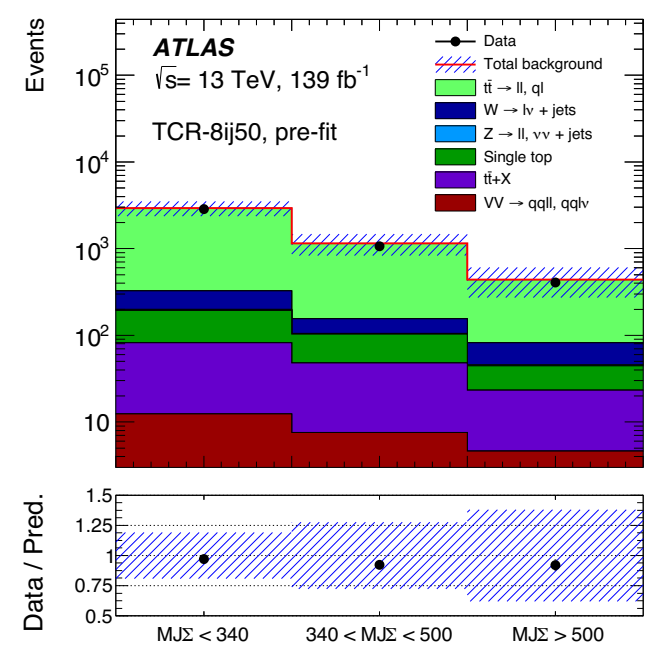

(b) TCR, pre-fit

Figure 4. Pre-fit yields in the (a) $W+$ jets and (b) $t \bar{t}$ background normalisation regions for the SR-8ij50 multi-bin analysis. The upper panel shows the absolute yields for data (black points) and all background subcomponents (histograms), with the combination of statistical and systematic uncertainties shown by the hatched areas. The lower panel shows the ratio of the data yields to the total SM prediction.

the signal, control and validation region selections, but assessed separately for each background process.

Additional uncertainties account for potential inaccuracies in the data-driven multijet estimate. The effects of residual kinematic correlations are estimated by modifying the $H_{\mathrm{T}}$-binning procedure used in the multijet estimate. A comparison between the nominal prediction and an alternative prediction assuming a broader resolution for flavour-tagged jets measured in data is used to estimate the impact of different flavour composition in 


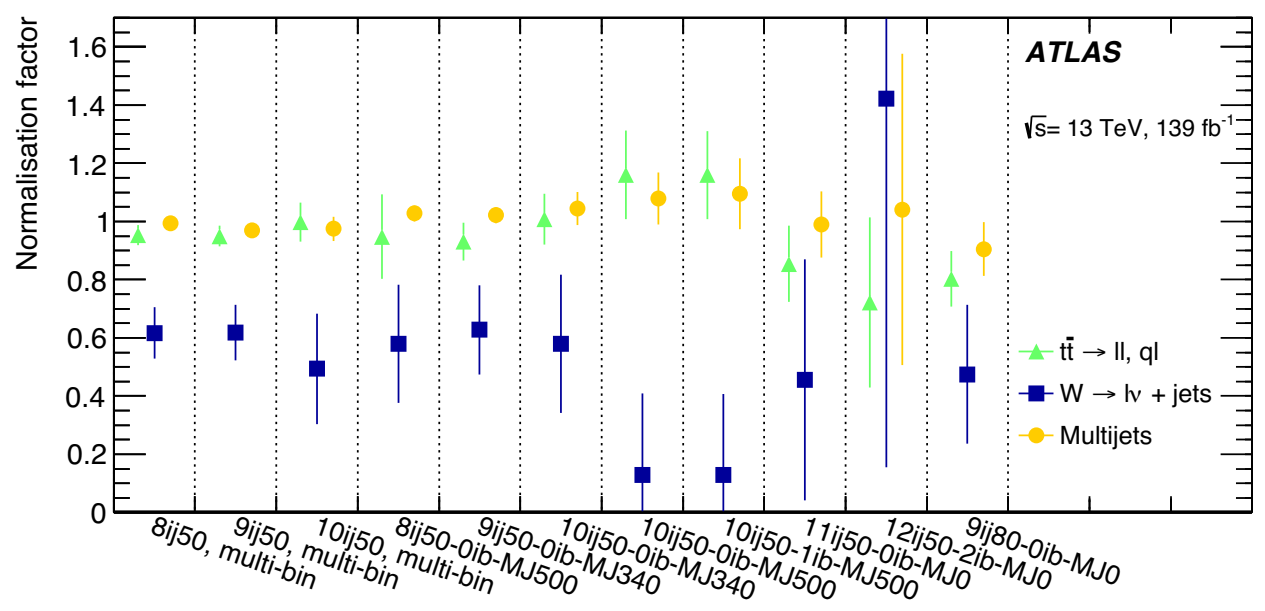

Figure 5. Summary of the fitted normalisation factors for the $t \bar{t}, W+$ jets and multijet backgrounds in all signal regions. The error bars indicate the combination of statistical and systematic uncertainties in the corresponding factors. Two pairs of SRs, namely the SR-8ij50 and SR-9ij50 multi-bin regions and the SR-10ij50-0ib-MJ500 and SR-10ij50-1ib-MJ500 single-bin regions share leptonic control regions, and therefore have highly correlated normalisation factors for the $W+$ jets and $t \bar{t}$ background components.

the template and signal regions. Finally, a conservative uncertainty is assessed from the observed non-closure of the prediction in validation regions.

The dominant sources of systematic uncertainty are listed in table 8. The total uncertainty in the predicted signal region yield is in the range $6-14 \%$ for the multi-bin signal regions, and 7-66\% for the less constrained single-bin regions. In both cases the largest uncertainties arise in the regions with the highest requirements on the jet multiplicity (and in the single-bin case also on the $b$-jet multiplicity or $M_{\mathrm{J}}^{\Sigma}$ ) since in those cases the supporting measurements are more statistically limited and so provide less precise constraints on the background predictions.

In each of the three multi-bin signal regions the most important uncertainty is the closure systematic uncertainty associated with the multijet template estimate (3-6\%), chiefly because of the dominance of this background component. In the single-bin signal regions the background normalisations are less constrained by the fit, and the statistical uncertainties associated with the multijet and $t \bar{t}$ control regions tend to dominate $(4-27 \%$ for multijets, $6-14 \%$ for $t \bar{t}$ ).

The theoretical uncertainty in the $t \bar{t}$ background predictions is also found to be significant, with that from final-state radiation contributing up to $17 \%$ in the single-bin fits. The impact on the total yield in multi-bin signal regions is $\leq 5 \%$. The $Z+$ jets component has a theoretical uncertainty of up to $14 \%$ in the single-bin regions while it is at most $6 \%$ in the multi-bin signal regions.

Most experimental systematic uncertainties affecting the reconstructed objects have insignificant impacts, being substantially reduced due to correlations between the MCbased predictions and the data-driven multijet template. The largest observed effects are 


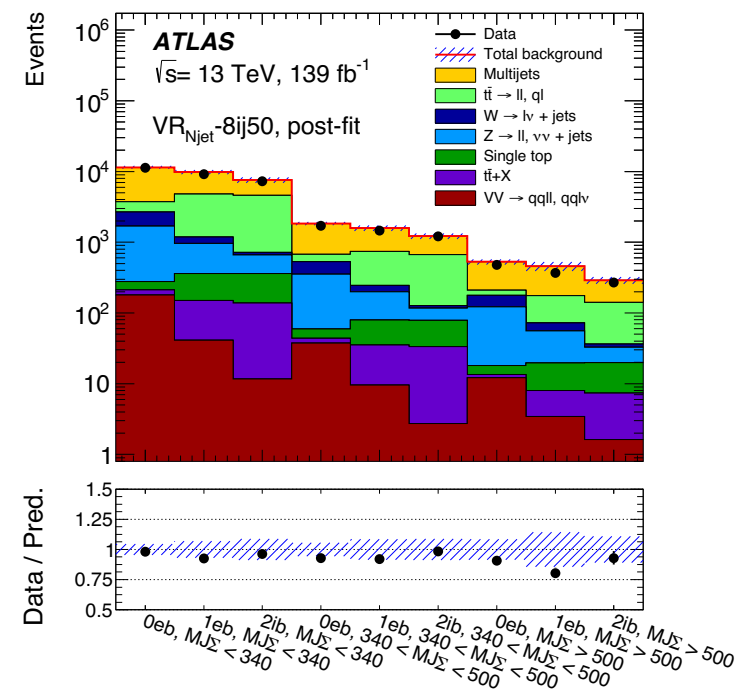

(a) $\operatorname{VR}_{N_{\text {jet }}}$

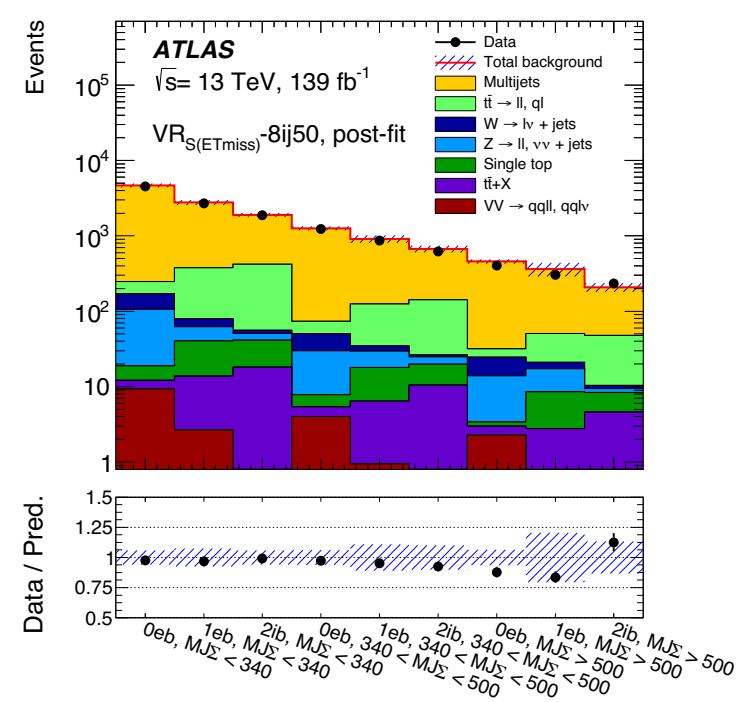

(b) $\operatorname{VR}_{\mathcal{S}\left(E_{T}^{\text {miss }}\right)}$

Figure 6. Post-fit event yields in validation regions for the SR-8ij50 multi-bin analysis. The upper panel shows the absolute yields for data (black points) and all background subcomponents (histograms), with the combination of statistical and systematic uncertainties shown by the hatched areas. The lower panel shows the ratio of the data yields to the total SM prediction. 


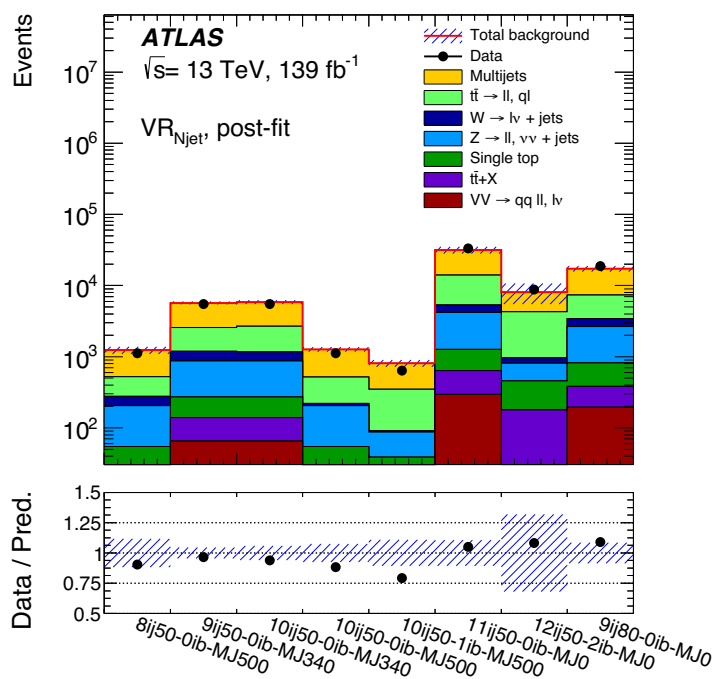

(a) $\operatorname{VR}_{N_{\text {jet }}}$

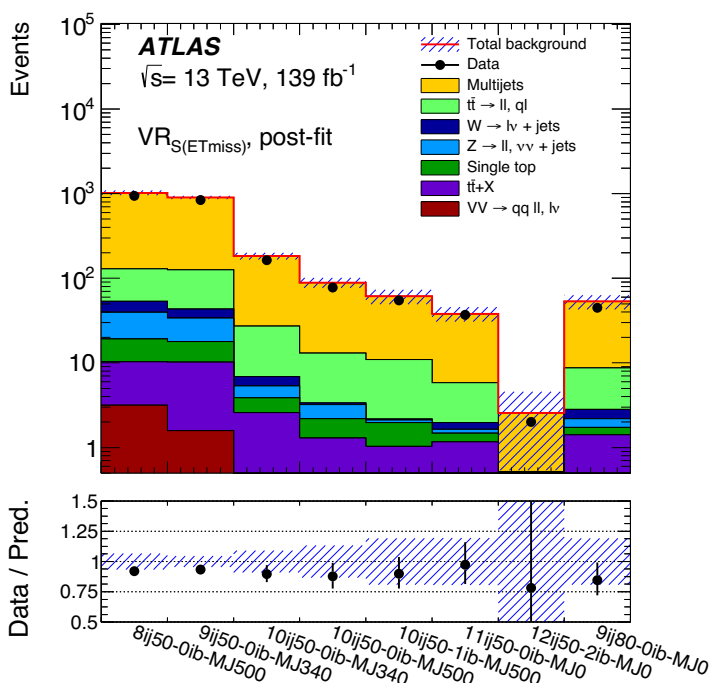

(b) $\operatorname{VR}_{\mathcal{S}\left(E_{T}^{\mathrm{miss}}\right)}$

Figure 7. Post-fit event yields in validation regions summarised for all single-bin signal regions. The upper panel shows the absolute yields for data (black points) and all background subcomponents (histograms), with the combination of statistical and systematic uncertainties shown by the hatched areas. The lower panel shows the ratio of the data yields to the total SM prediction. 


\begin{tabular}{|l|c|lr|lr|lr|}
\hline Signal region & Total syst. & \multicolumn{7}{|c|}{ Dominant systematic uncertainties } \\
\hline SR-8ij50-0ib-MJ500 & $16 \%$ & $E_{\mathrm{T}}^{\text {miss }}$ soft, L & $7 \%$ & $E_{\mathrm{T}}^{\text {miss }}$ soft, T & $7 \%$ & $Z$ +jets PS & $5 \%$ \\
SR-9ij50-0ib-MJ340 & $16 \%$ & $E_{\mathrm{T}}^{\text {miss }}$ soft, T & $9 \%$ & $E_{\mathrm{T}}^{\text {miss }}$ soft, L & $9 \%$ & $Z+$ jets PS & $4 \%$ \\
SR-10ij50-0ib-MJ340 & $20 \%$ & $t \bar{t}$ FSR & $9 \%$ & MC stat. & $9 \%$ & $E_{\mathrm{T}}^{\text {miss }}$ soft, L & $8 \%$ \\
SR-10ij50-0ib-MJ500 & $27 \%$ & $t \bar{t}$ FSR & $17 \%$ & MC stat. & $12 \%$ & $E_{\mathrm{T}}^{\text {miss }}$ soft, L & $9 \%$ \\
SR-10ij50-1ib-MJ500 & $24 \%$ & MC stat. & $14 \%$ & $E_{\mathrm{T}}^{\text {miss }}$ soft, L & $10 \%$ & $E_{\mathrm{T}}^{\text {miss }}$ soft, T & $10 \%$ \\
SR-11ij50 & $27 \%$ & MC stat. & $18 \%$ & $t \bar{t}$ FSR & $14 \%$ & $t \bar{t}$ norm & $6 \%$ \\
SR-12ij50-2ib & $70 \%$ & MC stat. & $62 \%$ & MJ norm & $25 \%$ & MJ $H_{\mathrm{T}}$ binning & $13 \%$ \\
SR-9ij80 & $21 \%$ & MC stat. & $14 \%$ & $Z+$ jets PS & $14 \%$ & $Z+$ jets match & $7 \%$ \\
\hline SR-8ij50 multi-bin & $6 \%$ & MJ closure & $3 \%$ & JES flavour & $3 \%$ & JES flavour & $2 \%$ \\
SR-9ij50 multi-bin & $7 \%$ & MJ closure & $4 \%$ & $Z$ +jets PS & $3 \%$ & $E_{\mathrm{T}}^{\text {miss }}$ soft, L & $3 \%$ \\
SR-10ij50 multi-bin & $14 \%$ & $Z+$ jets PS & $6 \%$ & MJ closure & $6 \%$ & $t \bar{t}$ FSR & $5 \%$ \\
\hline
\end{tabular}

Table 8. The total systematic uncertainties are shown for each of the single-bin signal regions, and also for the multi-bin signal regions, together with the three dominant contributions for each. The individual uncertainties can be (anti-)correlated, and do not necessarily sum in quadrature to the total background uncertainty. For the multi-bin signal regions the uncertainties are those found after summing the expected yields over the corresponding $M_{\mathrm{J}}^{\Sigma}$ and $b$-jet multiplicity bins of table 2 . Within the table 'MC stat.' indicates the statistical uncertainty of the simulated event yield in the $\mathrm{SR}$, 'MJ' indicates the uncertainty in the multijet background, 'closure' indicates the uncertainty from the multijet template method closure, 'norm' is the result of statistical uncertainties from the CRs, 'JES flavour' indicates the effect of uncertainties in the jet energy scale due to differences between quark- and gluon-initiated jets, ' $E_{\mathrm{T}}^{\mathrm{miss}}$ soft, $\mathrm{L} / \mathrm{T}$ ' indicate two sources, longitudinal and transverse, of uncertainty in the soft component of the missing transverse momentum, 'MJ $H_{\mathrm{T}}$ binning' relates to the parameters of the binning of the multijet template in $H_{\mathrm{T}}$, 'FSR' indicates final-state radiation, 'match' indicates the matrix element/parton shower matching scale, and 'PS' is the uncertainty from varying the scale at which the strong coupling constant is calculated for parton shower emissions in the MC simulation.

due to the uncertainties in the jet energy scale, which can have an effect of up to $3 \%$ due to the large jet activity in the events selected by this analysis, and in the soft term of the missing transverse momentum $(\leq 10 \%)$. In the least populated SRs, there can be a large statistical uncertainty in the predictions from simulation.

\section{$7 \quad$ Results and interpretation}

Data yields are shown graphically for all signal regions in figure 8. The background predicted to have the largest yield in all signal regions comes from multijet production. The relative contribution of the remaining backgrounds depends on the signal region. The $t \bar{t}$ process generally provides the second-largest contribution, and tends to form a larger fraction of the total background for higher jet-multiplicity requirements. As the requirement on the number of $b$-tagged jets increases, it can be seen that the relative contributions of $W+$ jets, $Z+$ jets and multi-boson backgrounds decrease compared to those from $t \bar{t}$ and single-top-quark production processes.

A breakdown of the yields in the single-bin regions is given in table 9. For illustration, the full $\mathcal{S}\left(E_{\mathrm{T}}^{\text {miss }}\right)$ distributions for several signal regions are shown in figure 9 . The 
data yields are found to be consistent with the background predictions within the assessed statistical and systematic uncertainties, with no significant excesses over the SM expectation. Mild deviations from the SM expectation are observed, with a tendency for the background to be overestimated in the higher jet-multiplicity regions, which is consistent with the trends observed in the corresponding validation regions. For interpretation, the likelihood fits for background estimation (section 6.3) are extended to include the signal region, and thereby perform two forms of hypothesis test using a profile-likelihood-ratio test statistic [90], quantifying the significance of any observed excesses or the lack thereof.

Firstly, the discovery test discriminates between the null hypothesis stating that the SR measurement is consistent with only SM contributions and an alternative hypothesis postulating a positive signal. Secondly, assuming a specific signal model, one may also form an exclusion test of the signal-plus-background hypothesis, where an observation significantly smaller than the combination of SM and SUSY processes would lead to rejection of the signal model. This provides the exclusion $p$-value $p_{1}$, the probability of observing at most the observed event yield when assuming that the signal is present with its nominal crosssection. A complementary $p$-value for the background observation $\mathrm{CL}_{\mathrm{b}}$ is defined as the probability of observing at most the observed yield under the background-only hypothesis. Points in the SUSY parameter space are considered excluded if the $\mathrm{CL}_{\mathrm{s}}$ parameter [91], computed as $p_{1} /\left(1-\mathrm{CL}_{\mathrm{b}}\right)$ is smaller than 0.05 . This protects against spurious exclusion of signals due to observing SR event counts significantly smaller than those predicted. While not strictly defining a frequentist confidence level, these are referred to as $95 \%$ confidence level (CL) limits.

The impact of the particle-flow reconstruction was assessed in the single-bin signal regions by applying the same event selection but instead using calorimeter-based hadronic reconstruction. As a result of the improved jet and $E_{\mathrm{T}}^{\text {miss }}$ resolution, the multijet background is found to be reduced by $30-50 \%$, corresponding to two standard deviations when considering statistical uncertainties as well as the systematic uncertainties of the jet energy scale and multijet estimate closure, resulting in an overall $20 \%$ lower total background yield in most regions. Consequently, the expected sensitivity to new physics signals is improved by up to $30 \%$ (one standard deviation), quantified in terms of the upper limit on BSM events.

The single-bin signal region event yields are used to derive model-independent constraints on the production of BSM particles. Table 10 shows the observed 95\% CL limits on the visible cross-section $\langle\epsilon \sigma\rangle_{\text {obs }}^{95}$ as well as the observed (expected) limits on the number of BSM signal events $S_{\text {obs }}^{95}\left(S_{\text {exp }}^{95}\right)$ in each signal region. The discovery $p$-value $p(s=0)$, defined as the probability of observing at least the observed event yield when assuming that no signal is present, is calculated, as is the corresponding Gaussian significance $Z$. For signal regions in which the predictions exceed the data, the value of $p(s=0)$ is capped at 0.5 . The smallest background $p$-value $\mathrm{CL}_{\mathrm{b}}$ computed in any region is 0.16 , while the smallest discovery $p$-value is 0.41 , and hence all observations are compatible with the SMonly hypothesis. The most stringent limits observed are for the SR-12ij50-2ib selection, for which visible cross-sections greater than $40 \mathrm{ab}$ are excluded.

Constraints on sparticle production in several benchmark parameter planes are shown in figure 10. These limits extend beyond those achieved by the previous search [16]. All 


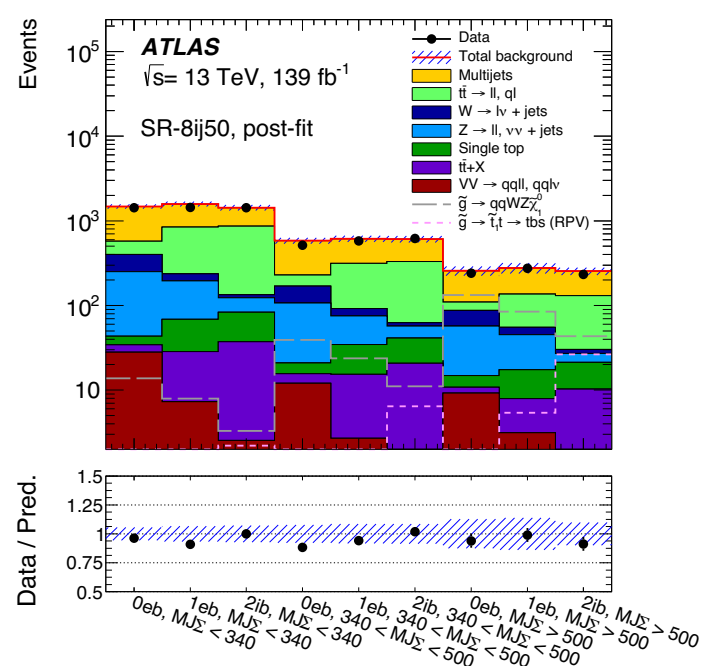

(a) SR-8ij50, multi-bin

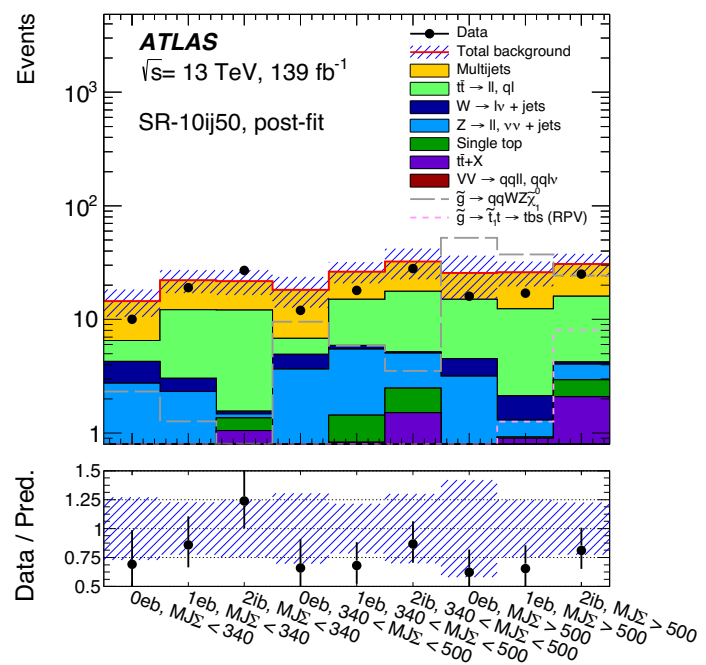

(c) SR-10ij50, multi-bin

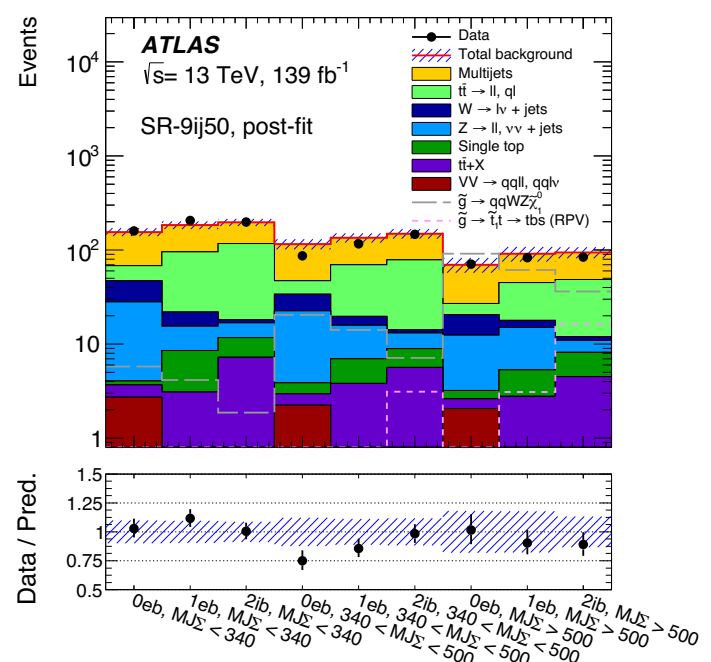

(b) SR-9ij50, multi-bin
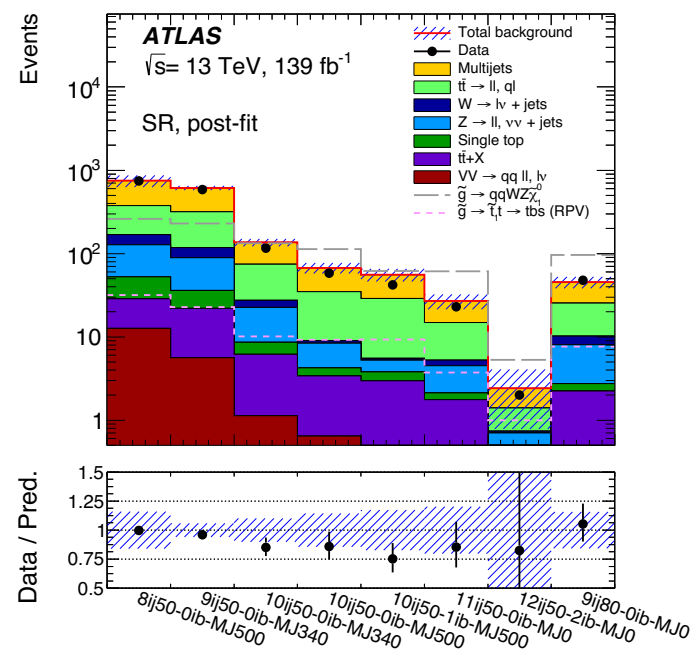

(d) Single-bin summary

Figure 8. Event yields in all signal regions, comparing data with the post-fit background predictions. The upper panel shows the absolute yields for data (black points) and all background subcomponents (histograms), with the combination of statistical and systematic uncertainties shown by the hatched areas. The yields for two benchmark signal models are overlaid, representing $1.6 \mathrm{TeV}$ gluinos decaying into $W$ and $Z$ bosons and a $100 \mathrm{GeV}$ neutralino via intermediate gauginos (long dashed histogram) or instead into $t b s / t b d$ via a $600 \mathrm{GeV}$ top squark through an $R$-parity-violating (RPV) coupling (short dashed histogram). The lower panel shows the ratio of the data yields to the total SM prediction. 


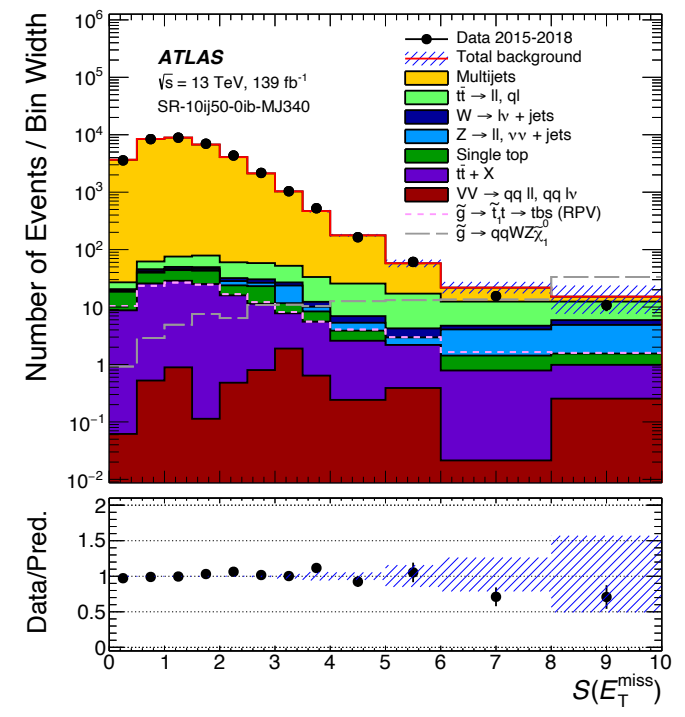

(a) SR-10ij50-0ib-MJ340
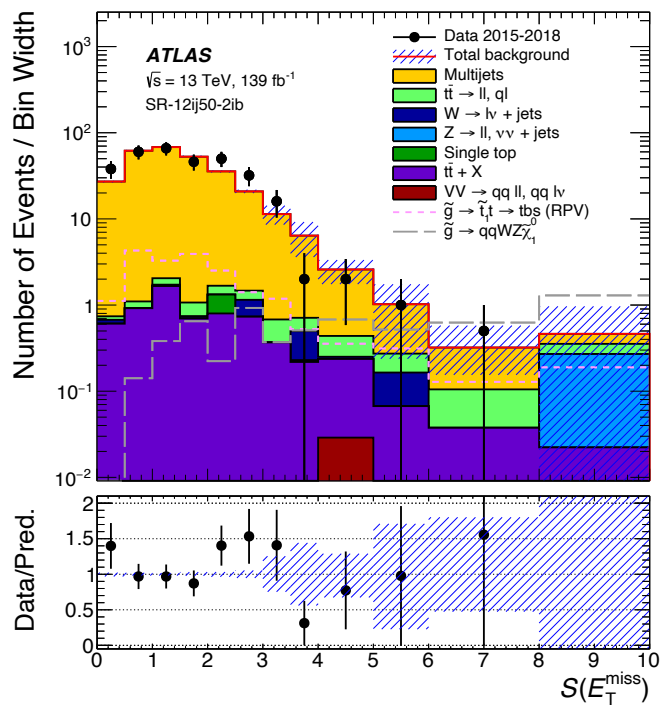

(b) SR-12ij50-2ib

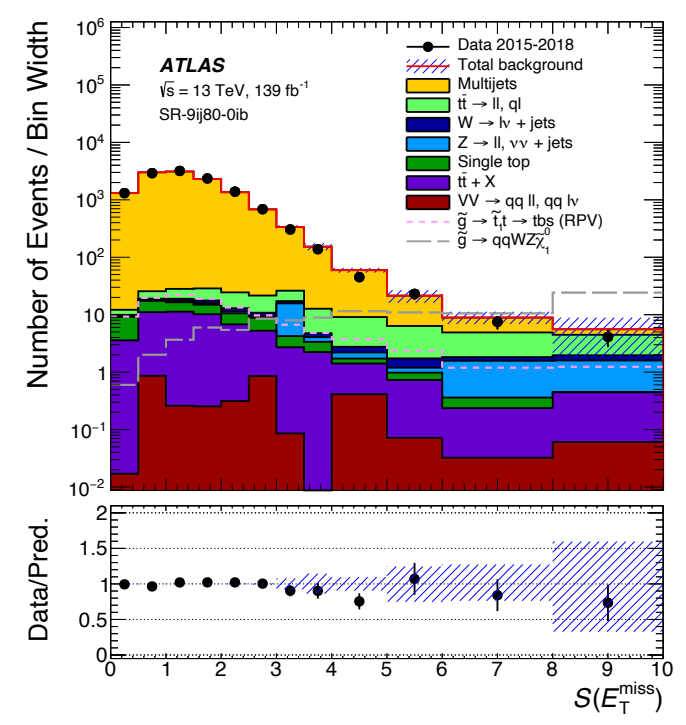

(c) SR-9ij80

Figure 9. Distribution of $\mathcal{S}\left(E_{\mathrm{T}}^{\mathrm{miss}}\right)$ in selected signal regions. The $t \bar{t}$ and $W+$ jets backgrounds are normalised according to the background fits, and the multijet background prediction adjusted accordingly. The upper panel shows the absolute yields for data (black points) and all background subcomponents (histograms), with the combination of statistical and systematic uncertainties shown by the hatched areas. The yields for two benchmark signal models are overlaid, representing $1.6 \mathrm{TeV}$ gluinos decaying into $W$ and $Z$ bosons and a $100 \mathrm{GeV}$ neutralino via intermediate gauginos (long dashed histogram) or instead into $t b s / t b d$ via a $600 \mathrm{GeV}$ top squark through an $R$-parity-violating (RPV) coupling (short dashed histogram). The lower panel shows the ratio of the data yields to the total SM prediction. 


\begin{tabular}{|lcc|}
\hline Background process & SR-8ij50-0ib-MJ500 & SR-9ij50-0ib-MJ340 \\
\hline Multijets & $372 \pm 46$ & $296 \pm 39$ \\
Top quark pairs & $208 \pm 89$ & $201 \pm 65$ \\
$W+$ jets & $41 \pm 20$ & $29 \pm 12$ \\
$Z+$ jets & $75 \pm 46$ & $53 \pm 33$ \\
Single top quarks & $23.9 \pm 10.5$ & $14.1 \pm 5.3$ \\
$t \bar{t}+X$ & $16.1 \pm 8.3$ & $16.4 \pm 8.4$ \\
Diboson, triboson & $12.7 \pm 1.7$ & $5.6 \pm 1.3$ \\
\hline Total background & $750 \pm 120$ & $614 \pm 97$ \\
Observed events & 747 & 588 \\
\hline
\end{tabular}

\begin{tabular}{|lccc|}
\hline Background process & SR-10ij50-0ib-MJ340 & SR-10ij50-0ib-MJ500 & SR-10ij50-1ib-MJ500 \\
\hline Multijets & $62 \pm 11$ & $32.8 \pm 6.1$ & $26.9 \pm 6.8$ \\
Top quark pairs & $47 \pm 17$ & $26 \pm 15$ & $23.5 \pm 9.8$ \\
$W+$ jets & $4.9 \pm 2.7$ & $0.6_{-0.6}^{+1.0}$ & $0.3_{-0.3}^{+0.4}$ \\
$Z+$ jets & $14 \pm 13$ & $4 \pm 4$ & $1.5_{-1.5}^{+1.6}$ \\
Single top quarks & $2.4 \pm 1.7$ & $0.9_{-0.9}^{+1.7}$ & $0.8_{-0.8}^{+1.1}$ \\
$t \bar{t}+X$ & $5.0 \pm 2.6$ & $2.7 \pm 1.5$ & $2.7 \pm 1.5$ \\
Diboson, triboson & $1.13 \pm 0.24$ & $0.65 \pm 0.19$ & $0.26 \pm 0.06$ \\
\hline Total background & $136 \pm 28$ & $68 \pm 18$ & $56 \pm 13$ \\
Observed events & 116 & 58 & 42 \\
\hline
\end{tabular}

\begin{tabular}{|lccc|}
\hline Background process & SR-11ij50 & SR-12ij50-2ib & SR-9ij80 \\
\hline Multijets & $12.0 \pm 3.8$ & $1.03 \pm 0.99$ & $20.0 \pm 5.1$ \\
Top quark pairs & $9.7 \pm 4.5$ & $0.66 \pm 0.53$ & $15.3 \pm 4.0$ \\
$W+$ jets & $0.75 \pm 0.75$ & $0.04_{-0.04}^{+0.08}$ & $2.2 \pm 1.2$ \\
$Z+$ jets & $2.4 \pm 1.4$ & $0.50 \pm 0.31$ & $5.3_{-5.3}^{+5.4}$ \\
Single top quarks & $0.4_{-0.4}^{+1.6}$ & $0.00 \pm 0.00$ & $0.50_{-0.50}^{+0.71}$ \\
$t \bar{t}+X$ & $1.4 \pm 0.8$ & $0.20 \pm 0.19$ & $1.9 \pm 1.0$ \\
Diboson, triboson & $0.31 \pm 0.06$ & $0.00 \pm 0.00$ & $0.32 \pm 0.06$ \\
\hline Total background & $27.0 \pm 7.3$ & $2.4 \pm 1.7$ & $45.6 \pm 9.5$ \\
Observed events & 23 & 2 & 48 \\
\hline
\end{tabular}

Table 9. Detailed event yields in all single-bin signal regions. Due to correlations, summing the uncertainties of individual background components may not reproduce the final uncertainty of the total background prediction.

exclusion fits are consistent with the background-only hypothesis, considering the total uncertainties.

For all three signal scenarios, the multi-bin limits provide the strongest expected constraints. There are two exceptions: the SR-9ij80-0ib selection reaches parity with the multi-bin limits for small neutralino masses in the two-step gluino decay model, while at low gluino masses in the RPV decay the SR-8ij50-0ib-MJ500 single-bin region achieves better sensitivity for small mass-splittings, but only for signal models already excluded 


\begin{tabular}{|lccrcc|}
\hline Signal channel & $\langle\epsilon \sigma\rangle_{\text {obs }}^{95}[\mathrm{fb}]$ & \multicolumn{1}{c}{$S_{\text {obs }}^{95}$} & \multicolumn{1}{c|}{$S_{\text {exp }}^{95}$} & $\mathrm{CL}_{\mathrm{b}}$ & $p(s=0)(Z)$ \\
\hline SR-8ij50-0ib-MJ500 & 1.16 & 163 & $162_{-39}^{+33}$ & 0.50 & $0.50(0.00)$ \\
SR-9ij50-0ib-MJ340 & 0.95 & 133 & $140_{-31}^{+30}$ & 0.50 & $0.50(0.00)$ \\
SR-10ij50-0ib-MJ340 & 0.22 & 31 & $40_{-11}^{+15}$ & 0.21 & $0.50(0.00)$ \\
SR-10ij50-0ib-MJ500 & 0.16 & 21.9 & $25.9_{-6.8}^{+9.6}$ & 0.29 & $0.50(0.00)$ \\
SR-10ij50-1ib-MJ500 & 0.12 & 16.8 & $22.8_{-6.1}^{+8.5}$ & 0.16 & $0.50(0.00)$ \\
SR-11ij50 & 0.09 & 13.0 & $15.1_{-4.2}^{+6.0}$ & 0.32 & $0.50(0.00)$ \\
SR-12ij50-2ib & 0.04 & 5.0 & $5.2_{-1.7}^{+2.5}$ & 0.44 & $0.50(0.00)$ \\
SR-9ij80 & 0.18 & 25.2 & $24.5_{-6.3}^{+7.0}$ & 0.57 & $0.41(0.22)$ \\
\hline
\end{tabular}

Table 10. Left to right: $95 \%$ CL upper limits on the visible cross-section $\left(\langle\epsilon \sigma\rangle_{\text {obs }}^{95}\right)$ and on the number of signal events $\left(S_{\mathrm{obs}}^{95}\right)$. The third column $\left(S_{\text {exp }}^{95}\right)$ shows the $95 \% \mathrm{CL}$ upper limit on the number of signal events, given the expected number of background events (and the $\pm 1 \sigma$ excursions around the expectation). The last two columns indicate the $\mathrm{CL}_{\mathrm{b}}$ value, i.e. the confidence level observed for the background-only hypothesis, and the discovery $p$-value $(p(s=0))$, together with the corresponding Gaussian significance $(Z)$.

by previous searches. More significantly, the multi-bin approach enhances the sensitivity to RPV decays of the gluino, as well as to gluino-mediated stop production in the region where the LSP is moderately massive. The 8-jet multi-bin region is most sensitive where the gluino-neutralino mass-splitting is smaller, while the 9- and 10-jet selections take over as the mass-splitting increases, which allows better signal-background discrimination with a harsher selection.

The strongest bounds on the gluino mass are placed at $m_{\tilde{g}}=2 \mathrm{TeV}$ for gluinos decaying via a two-step cascade to $W$ and $Z$ bosons when assuming nearly massless neutralinos. For gluino decays into a $t \bar{t}$ pair and $E_{\mathrm{T}}^{\text {miss }}$ via $\mathrm{RPC}$ couplings, the region with $m_{\tilde{g}}<1.8 \mathrm{TeV}$ and $m_{\tilde{\chi}_{1}^{0}}<700 \mathrm{GeV}$ is excluded, with the highest excluded neutralino mass being $m_{\tilde{\chi}_{1}^{0}} \simeq$ $950 \mathrm{GeV}$. This represents an improvement of $260 \mathrm{GeV}$ in $m_{\tilde{g}}$ and $230 \mathrm{GeV}$ in $m_{\tilde{\chi}_{1}^{0}}$ with respect to the previous search results. In the case of stop-mediated gluino decays via RPV couplings, the reach in $m_{\tilde{g}}$ is above $1.5 \mathrm{TeV}$ for $\tilde{t}_{1}$ masses of $400 \mathrm{GeV}$ to $1.1 \mathrm{TeV}$, with the maximal reach being achieved almost $1.6 \mathrm{TeV}$ for $m_{\tilde{t}_{1}}=900 \mathrm{GeV}$.

\section{Conclusion}

A search for new physics, producing many jets and moderate $E_{\mathrm{T}}^{\text {miss }}$, is presented, using the $139 \mathrm{fb}^{-1}$ dataset of $13 \mathrm{TeV} p p$ collisions collected by ATLAS during Run 2 of the LHC. The analysis selects events in regions with large jet multiplicities (from $\geq 8$ to $\geq 12$ jets) with further requirements on the number of $b$-tagged jets, and on the sum of the masses of largeradius jets. This search is the first from ATLAS to exploit particle-flow reconstruction for jets and $E_{\mathrm{T}}^{\mathrm{miss}}$. The combination of this improvement together with a better object-based $\mathcal{S}\left(E_{\mathrm{T}}^{\mathrm{miss}}\right)$ definition and a multi-bin statistical analysis leads to significant improvements in the search sensitivity beyond that afforded simply by the larger dataset.

No significant deviations from the Standard Model expectations are observed, while limits on the production of new particles are significantly extended. In the context of $R$ - 


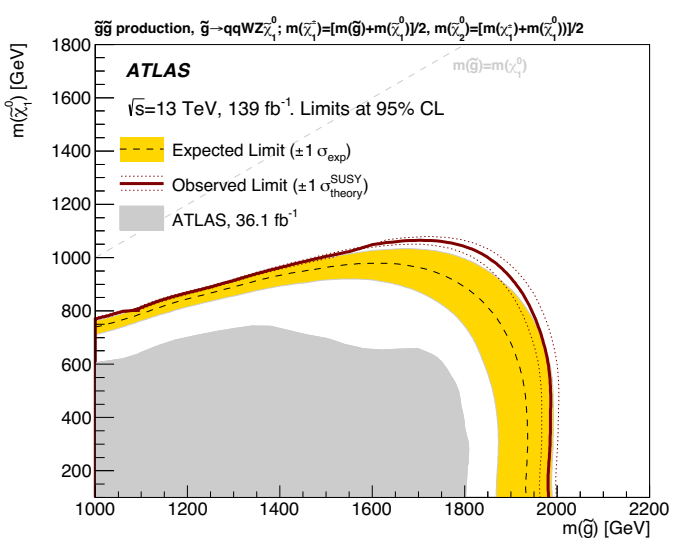

(a) $\tilde{g}, 2$-step cascade

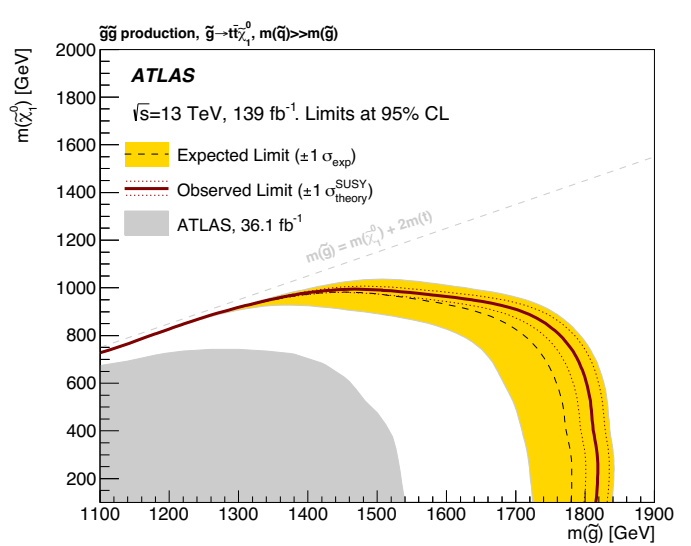

(b) $\tilde{g} \rightarrow t \bar{t} \tilde{\chi}_{1}^{0}$

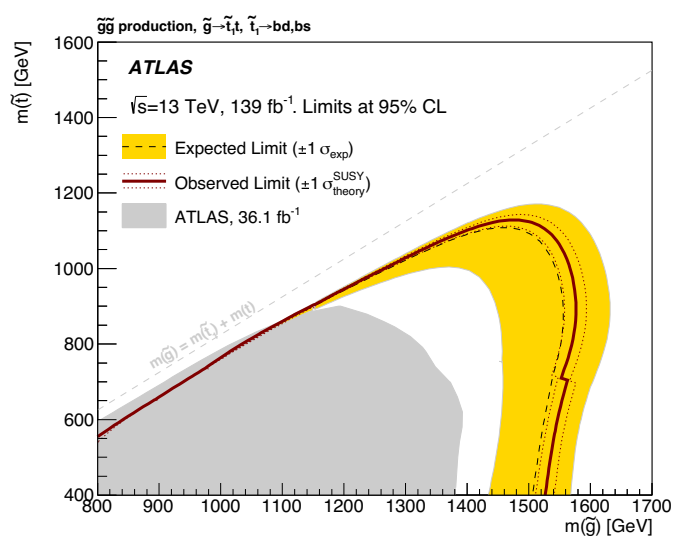

(c) RPV $\tilde{g} \rightarrow t b d, t b s$

Figure 10. Exclusion limits on gluino production in various parameter planes. The solid maroon line indicates the observed exclusion limit at $95 \% \mathrm{CL}$. Transitions between two signal regions which provide the best expected sensitivity in different regions of the parameter space may lead to discontinuities in the observed limit. The $1 \sigma$ variations in the observed limit due to the uncertainty on the signal production cross-sections are shown with dotted maroon lines. A dashed black line and yellow band respectively indicate the expected limit and its $1 \sigma$ variations due to all uncertainties in the signal acceptance and background yields. Grey shading is used to indicate the observed limit from the previous publication [16].

parity conserving supersymmetry, limits on the gluino mass reach $2 \mathrm{TeV}$ in the case of a two-step cascade via vector bosons, which are the most stringent limits observed to date when assuming a low-mass $\tilde{\chi}_{1}^{0}$. For gluino-pair production followed by the decay $\tilde{g} \rightarrow t \bar{t} \tilde{\chi}_{1}^{0}$ gluino masses up to $1.8 \mathrm{TeV}$ are excluded for $\tilde{\chi}_{1}^{0}$ masses up to $700 \mathrm{GeV}$. In the case of gluino-pair production followed by the $R$-parity-violating decays, $\tilde{g} \rightarrow t b d$, $t b s$, limits extend up to almost $1.6 \mathrm{TeV}$ for a $\tilde{t}$ mass of $900 \mathrm{GeV}$. Model-independent upper limits on the visible cross-section are set as low as $40 \mathrm{ab}$, in signal regions that probe extremely large jet multiplicities, with the most stringent selections requiring $\geq 12$ jets of which two are $b$-tagged. 


\section{Acknowledgments}

We thank CERN for the very successful operation of the LHC, as well as the support staff from our institutions without whom ATLAS could not be operated efficiently.

We acknowledge the support of ANPCyT, Argentina; YerPhI, Armenia; ARC, Australia; BMWFW and FWF, Austria; ANAS, Azerbaijan; SSTC, Belarus; CNPq and FAPESP, Brazil; NSERC, NRC and CFI, Canada; CERN; CONICYT, Chile; CAS, MOST and NSFC, China; COLCIENCIAS, Colombia; MSMT CR, MPO CR and VSC CR, Czech Republic; DNRF and DNSRC, Denmark; IN2P3-CNRS and CEA-DRF/IRFU, France; SRNSFG, Georgia; BMBF, HGF and MPG, Germany; GSRT, Greece; RGC and Hong Kong SAR, China; ISF and Benoziyo Center, Israel; INFN, Italy; MEXT and JSPS, Japan; CNRST, Morocco; NWO, Netherlands; RCN, Norway; MNiSW and NCN, Poland; FCT, Portugal; MNE/IFA, Romania; MES of Russia and NRC KI, Russia Federation; JINR; MESTD, Serbia; MSSR, Slovakia; ARRS and MIZŠ, Slovenia; DST/NRF, South Africa; MICINN, Spain; SRC and Wallenberg Foundation, Sweden; SERI, SNSF and Cantons of Bern and Geneva, Switzerland; MOST, Taiwan; TAEK, Turkey; STFC, United Kingdom; DOE and NSF, United States of America. In addition, individual groups and members have received support from BCKDF, CANARIE, Compute Canada and CRC, Canada; ERC, ERDF, Horizon 2020, Marie Skłodowska-Curie Actions and COST, European Union; Investissements d'Avenir Labex, Investissements d'Avenir Idex and ANR, France; DFG and AvH Foundation, Germany; Herakleitos, Thales and Aristeia programmes co-financed by EU-ESF and the Greek NSRF, Greece; BSF-NSF and GIF, Israel; La Caixa Banking Foundation, CERCA Programme Generalitat de Catalunya and PROMETEO and GenT Programmes Generalitat Valenciana, Spain; Göran Gustafssons Stiftelse, Sweden; The Royal Society and Leverhulme Trust, United Kingdom.

The crucial computing support from all WLCG partners is acknowledged gratefully, in particular from CERN, the ATLAS Tier-1 facilities at TRIUMF (Canada), NDGF (Denmark, Norway, Sweden), CC-IN2P3 (France), KIT/GridKA (Germany), INFN-CNAF (Italy), NL-T1 (Netherlands), PIC (Spain), ASGC (Taiwan), RAL (U.K.) and BNL (U.S.A.), the Tier-2 facilities worldwide and large non-WLCG resource providers. Major contributors of computing resources are listed in ref. [92].

Open Access. This article is distributed under the terms of the Creative Commons Attribution License (CC-BY 4.0), which permits any use, distribution and reproduction in any medium, provided the original author(s) and source are credited.

\section{References}

[1] L. Evans and P. Bryant, eds., LHC machine, 2008 JINST 3 S08001 [INSPIRE].

[2] Y. Golfand and E.P. Likhtman, Extension of the Algebra of Poincaré Group Generators and Violation of p Invariance, JETP Lett. 13 (1971) 323 [Pisma Zh. Eksp. Teor. Fiz. 13 (1971) 452] [INSPIRE].

[3] D.V. Volkov and V.P. Akulov, Is the Neutrino a Goldstone Particle?, Phys. Lett. B 46 (1973) 109 [INSPIRE]. 
[4] J. Wess and B. Zumino, Supergauge transformations in four-dimensions, Nucl. Phys. B $\mathbf{7 0}$ (1974) 39 [INSPIRE].

[5] J. Wess and B. Zumino, Supergauge invariant extension of quantum electrodynamics, Nucl. Phys. B 78 (1974) 1 [inSPIRE].

[6] S. Ferrara and B. Zumino, Supergauge invariant Yang-Mills theories, Nucl. Phys. B 79 (1974) 413.

[7] A. Salam and J.A. Strathdee, Supersymmetry and non-Abelian gauges, Phys. Lett. B 51 (1974) 353 [INSPIRE].

[8] G.R. Farrar and P. Fayet, Phenomenology of the production, decay, and detection of new hadronic states associated with supersymmetry, Phys. Lett. B 76 (1978) 575 [InSPIRE].

[9] H.D. Dreiner, An introduction to explicit R-parity violation, hep-ph/9707435.

[10] ATLAS collaboration, The ATLAS experiment at the CERN Large Hadron Collider, 2008 JINST 3 S08003 [INSPIRE].

[11] T. Cohen, E. Izaguirre, M. Lisanti and H.K. Lou, Jet substructure by accident, JHEP 03 (2013) 161 [arXiv:1212.1456] [INSPIRE].

[12] ATLAS collaboration, Search for new phenomena in final states with large jet multiplicities and missing transverse momentum using $\sqrt{s}=7 \mathrm{TeV}$ pp collisions with the ATLAS detector, JHEP 11 (2011) 099 [arXiv:1110.2299] [INSPIRE].

[13] ATLAS collaboration, Hunt for new phenomena using large jet multiplicities and missing transverse momentum with ATLAS in $4.7 \mathrm{fb}^{-1}$ of $\sqrt{s}=7 \mathrm{TeV}$ proton-proton collisions, JHEP 07 (2012) 167 [arXiv: 1206.1760] [INSPIRE].

[14] ATLAS collaboration, Search for new phenomena in final states with large jet multiplicities and missing transverse momentum at $\sqrt{s}=8$ TeV proton-proton collisions using the ATLAS experiment, JHEP 10 (2013) 130 [Erratum ibid. 01 (2014) 109] [arXiv:1308.1841] [INSPIRE].

[15] ATLAS collaboration, Search for new phenomena in final states with large jet multiplicities and missing transverse momentum with ATLAS using $\sqrt{s}=13$ TeV proton-proton collisions, Phys. Lett. B 757 (2016) 334 [arXiv: 1602.06194] [InSPIRE].

[16] ATLAS collaboration, Search for new phenomena with large jet multiplicities and missing transverse momentum using large-radius jets and flavour-tagging at ATLAS in $13 \mathrm{TeV} p p$ collisions, JHEP 12 (2017) 034 [arXiv:1708.02794] [INSPIRE].

[17] ATLAS collaboration, Jet reconstruction and performance using particle flow with the ATLAS Detector, Eur. Phys. J. C 77 (2017) 466 [arXiv:1703.10485] [INSPIRE].

[18] ATLAS collaboration, Object-based missing transverse momentum significance in the ATLAS detector, ATLAS-CONF-2018-038 (2018).

[19] ATLAS collaboration, ATLAS insertable B-layer technical design report, CERN-LHCC-2010-013 (2010).

[20] ATLAS IBL collaboration, Production and integration of the ATLAS insertable B-layer, 2018 JINST 13 T05008 [arXiv:1803.00844] [INSPIRE].

[21] ATLAS collaboration, Performance of the ATLAS Trigger System in 2015, Eur. Phys. J. C 77 (2017) 317 [arXiv:1611.09661] [INSPIRE]. 
[22] ATLAS collaboration, Luminosity determination in pp collisions at $\sqrt{s}=13 \mathrm{TeV}$ using the ATLAS detector at the LHC, ATLAS-CONF-2019-021 (2019).

[23] G. Avoni et al., The new LUCID-2 detector for luminosity measurement and monitoring in ATLAS, 2018 JINST 13 P07017 [INSPIRE].

[24] ATLAS collaboration, Trigger menu in 2017, ATL-DAQ-PUB-2018-002 (2018).

[25] ATLAS collaboration, ATLAS data quality operations and performance for 2015-2018 data-taking, 2020 JINST 15 P04003 [arXiv: 1911.04632] [INSPIRE].

[26] ATLAS collaboration, Characterisation and mitigation of beam-induced backgrounds observed in the ATLAS detector during the 2011 proton-proton run, 2013 JINST 8 P07004 [arXiv: 1303.0223] [INSPIRE].

[27] ATLAS collaboration, Selection of jets produced in $13 \mathrm{TeV}$ proton-proton collisions with the ATLAS detector, ATLAS-CONF-2015-029 (2015).

[28] T. Sjöstrand, S. Mrenna and P.Z. Skands, A brief introduction to PYTHIA 8.1, Comput. Phys. Commun. 178 (2008) 852 [arXiv:0710.3820] [INSPIRE].

[29] ATLAS collaboration, The PYTHIA 8 A3 tune description of ATLAS minimum bias and inelastic measurements incorporating the Donnachie-Landshoff diffractive model, Tech. Rep. ATL-PHYS-PUB-2016-017, CERN, Geneva (Aug, 2016).

[30] R.D. Ball et al., Parton distributions with LHC data, Nucl. Phys. B 867 (2013) 244 [arXiv:1207.1303] [INSPIRE].

[31] ATLAS collaboration, The ATLAS simulation infrastructure, Eur. Phys. J. C 70 (2010) 823 [arXiv: 1005.4568] [INSPIRE].

[32] GEANT4 collaboration, GEANT4 - a simulation toolkit, Nucl. Instrum. Meth. A 506 (2003) 250 [INSPIRE].

[33] ATLAS collaboration, Performance of the Fast ATLAS Tracking Simulation (FATRAS) and the ATLAS Fast Calorimeter Simulation (FastCaloSim) with single particles, ATL-SOFT-PUB-2014-001 (2014).

[34] P. Nason, A new method for combining NLO QCD with shower Monte Carlo algorithms, JHEP 11 (2004) 040 [hep-ph/0409146] [INSPIRE].

[35] S. Frixione, P. Nason and C. Oleari, Matching NLO QCD computations with Parton Shower simulations: the POWHEG method, JHEP 11 (2007) 070 [arXiv: 0709. 2092] [INSPIRE].

[36] S. Alioli, P. Nason, C. Oleari and E. Re, A general framework for implementing NLO calculations in shower Monte Carlo programs: the POWHEG BOX, JHEP 06 (2010) 043 [arXiv: 1002.2581] [INSPIRE].

[37] S. Alioli, P. Nason, C. Oleari and E. Re, NLO single-top production matched with shower in POWHEG: s- and t-channel contributions, JHEP 09 (2009) 111 [Erratum ibid. 02 (2010) 011] [arXiv:0907.4076] [INSPIRE].

[38] E. Re, Single-top Wt-channel production matched with parton showers using the POWHEG method, Eur. Phys. J. C 71 (2011) 1547 [arXiv:1009.2450] [INSPIRE].

[39] S. Alioli, S.-O. Moch and P. Uwer, Hadronic top-quark pair-production with one jet and parton showering, JHEP 01 (2012) 137 [arXiv:1110.5251] [INSPIRE].

[40] NNPDF collaboration, Parton distributions for the LHC Run II, JHEP 04 (2015) 040 [arXiv: 1410.8849] [INSPIRE]. 
[41] S. Frixione, E. Laenen, P. Motylinski, B.R. Webber and C.D. White, Single-top hadroproduction in association with a W boson, JHEP 07 (2008) 029 [arXiv:0805.3067] [INSPIRE].

[42] S. Frixione, E. Laenen, P. Motylinski and B.R. Webber, Angular correlations of lepton pairs from vector boson and top quark decays in Monte Carlo simulations, JHEP 04 (2007) 081 [hep-ph/0702198] [INSPIRE].

[43] P. Artoisenet, R. Frederix, O. Mattelaer and R. Rietkerk, Automatic spin-entangled decays of heavy resonances in Monte Carlo simulations, JHEP 03 (2013) 015 [arXiv:1212.3460] [INSPIRE].

[44] T. Sjöstrand et al., An introduction to PYTHIA 8.2, Comput. Phys. Commun. 191 (2015) 159 [arXiv: 1410.3012] [INSPIRE].

[45] ATLAS collaboration, ATLAS PYTHIA 8 tunes to 7 TeV datas, ATL-PHYS-PUB-2014-021 (2014).

[46] D.J. Lange, The EvtGen particle decay simulation package, Nucl. Instrum. Meth. A 462 (2001) 152 [INSPIRE].

[47] T. Gleisberg et al., Event generation with SHERPA 1.1, JHEP 02 (2009) 007 [arXiv: 0811.4622] [INSPIRE].

[48] M. Czakon and A. Mitov, Top++: a program for the calculation of the top-pair cross-section at hadron colliders, Comput. Phys. Commun. 185 (2014) 2930 [arXiv:1112.5675] [INSPIRE].

[49] N. Kidonakis, Two-loop soft anomalous dimensions for single top quark associated production with a $W$ - or H-, Phys. Rev. D 82 (2010) 054018 [arXiv: 1005.4451] [INSPIRE].

[50] N. Kidonakis, Top quark production, in the proceedings of the Helmholtz International Summer School on Physics of Heavy Quarks and Hadrons (HQ 2013), July 15-28, Dubna, Russia (2013), arXiv:1311.0283 [INSPIRE].

[51] M. Aliev, H. Lacker, U. Langenfeld, S. Moch, P. Uwer and M. Wiedermann, HATHOR: HAdronic Top and Heavy quarks crOss section calculatoR, Comput. Phys. Commun. 182 (2011) 1034 [arXiv: 1007.1327] [INSPIRE].

[52] P. Kant et al., HatHor for single top-quark production: updated predictions and uncertainty estimates for single top-quark production in hadronic collisions, Comput. Phys. Commun. 191 (2015) 74 [arXiv: 1406.4403] [INSPIRE].

[53] J. Alwall et al., The automated computation of tree-level and next-to-leading order differential cross sections, and their matching to parton shower simulations, JHEP 07 (2014) 079 [arXiv: 1405.0301] [INSPIRE].

[54] LHC Higgs Cross Section Working Group collaboration, Handbook of LHC Higgs Cross Sections: 4. Deciphering the Nature of the Higgs Sector, arXiv:1610.07922 [INSPIRE].

[55] T. Gleisberg and S. Hoeche, Comix, a new matrix element generator, JHEP 12 (2008) 039 [arXiv:0808.3674] [INSPIRE].

[56] F. Cascioli, P. Maierhofer and S. Pozzorini, Scattering amplitudes with open loops, Phys. Rev. Lett. 108 (2012) 111601 [arXiv:1111.5206] [InSPIRE].

[57] S. Schumann and F. Krauss, A parton shower algorithm based on Catani-Seymour dipole factorisation, JHEP 03 (2008) 038 [arXiv:0709.1027] [INSPIRE]. 
[58] S. Hoeche, F. Krauss, S. Schumann and F. Siegert, QCD matrix elements and truncated showers, JHEP 05 (2009) 053 [arXiv:0903.1219] [INSPIRE].

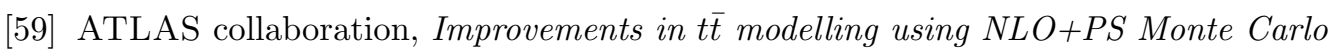
generators for Run2, ATL-PHYS-PUB-2018-009 (2018).

[60] M. Bahr et al., HERWIG++ physics and manual, Eur. Phys. J. C 58 (2008) 639 [arXiv: 0803.0883] [INSPIRE].

[61] J. Bellm et al., HERWIG 7.0/HERWIG++ 3.0 release note, Eur. Phys. J. C 76 (2016) 196 [arXiv: 1512.01178] [INSPIRE].

[62] L.A. Harland-Lang, A.D. Martin, P. Motylinski and R.S. Thorne, Parton distributions in the LHC era: MMHT 2014 PDFs, Eur. Phys. J. C 75 (2015) 204 [arXiv:1412.3989] [InSPIRE].

[63] ATLAS collaboration, Multi-boson simulation for $13 \mathrm{TeV}$ ATLAS analyses, ATL-PHYS-PUB-2017-005 (2017).

[64] ATLAS collaboration, ATLAS simulation of boson plus jets processes in Run 2, ATL-PHYS-PUB-2017-006 (2017).

[65] L. Lönnblad, Correcting the color dipole cascade model with fixed order matrix elements, JHEP 05 (2002) 046 [hep-ph/0112284] [INSPIRE].

[66] W. Beenakker, C. Borschensky, M. Krämer, A. Kulesza and E. Laenen, NNLL-fast: predictions for coloured supersymmetric particle production at the LHC with threshold and Coulomb resummation, JHEP 12 (2016) 133 [arXiv:1607.07741] [INSPIRE].

[67] W. Beenakker et al., NNLL resummation for squark and gluino production at the LHC, JHEP 12 (2014) 023 [arXiv: 1404.3134] [INSPIRE].

[68] W. Beenakker et al., Towards NNLL resummation: hard matching coefficients for squark and gluino hadroproduction, JHEP 10 (2013) 120 [arXiv:1304.6354] [INSPIRE].

[69] W. Beenakker, S. Brensing, M. Krämer, A. Kulesza, E. Laenen and I. Niessen, NNLL resummation for squark-antisquark pair production at the LHC, JHEP 01 (2012) 076 [arXiv:1110.2446] [INSPIRE].

[70] W. Beenakker, S. Brensing, M. Krämer, A. Kulesza, E. Laenen and I. Niessen, Soft-gluon resummation for squark and gluino hadroproduction, JHEP 12 (2009) 041 [arXiv:0909.4418] [INSPIRE].

[71] A. Kulesza and L. Motyka, Soft gluon resummation for the production of gluino-gluino and squark-antisquark pairs at the LHC, Phys. Rev. D 80 (2009) 095004 [arXiv:0905.4749] [INSPIRE].

[72] A. Kulesza and L. Motyka, Threshold resummation for squark-antisquark and gluino-pair production at the LHC, Phys. Rev. Lett. 102 (2009) 111802 [arXiv:0807.2405] [INSPIRE].

[73] W. Beenakker, R. Hopker, M. Spira and P.M. Zerwas, Squark and gluino production at hadron colliders, Nucl. Phys. B 492 (1997) 51 [hep-ph/9610490] [InSPIRE].

[74] J. Butterworth et al., PDF4LHC recommendations for LHC Run II, J. Phys. G 43 (2016) 023001 [arXiv: 1510.03865] [INSPIRE].

[75] ATLAS collaboration, Vertex reconstruction performance of the ATLAS detector at $\sqrt{s}=13 \mathrm{TeV}$, ATL-PHYS-PUB-2015-026 (2015).

[76] M. Cacciari, G.P. Salam and G. Soyez, The anti- $k_{t}$ jet clustering algorithm, JHEP 04 (2008) 063 [arXiv: 0802.1189] [INSPIRE]. 
[77] M. Cacciari, G.P. Salam and G. Soyez, FastJet user manual, Eur. Phys. J. C 72 (2012) 1896 [arXiv: 1111.6097] [INSPIRE].

[78] ATLAS collaboration, Topological cell clustering in the ATLAS calorimeters and its performance in LHC Run 1, Eur. Phys. J. C 77 (2017) 490 [arXiv:1603.02934] [INSPIRE].

[79] ATLAS collaboration, Performance of pile-up mitigation techniques for jets in pp collisions at $\sqrt{s}=8 \mathrm{TeV}$ using the ATLAS detector, Eur. Phys. J. C 76 (2016) 581 [arXiv: 1510.03823] [INSPIRE].

[80] ATLAS collaboration, Jet reclustering and close-by effects in ATLAS run II, ATLAS-CONF-2017-062 (2017).

[81] ATLAS collaboration, Optimisation of the ATLAS b-tagging performance for the 2016 LHC Run, ATL-PHYS-PUB-2016-012 (2016).

[82] ATLAS collaboration, ATLAS b-jet identification performance and efficiency measurement with $t \bar{t}$ events in pp collisions at $\sqrt{s}=13$ TeV, Eur. Phys. J. C 79 (2019) 970 [arXiv: 1907.05120] [INSPIRE].

[83] ATLAS collaboration, Electron and photon performance measurements with the ATLAS detector using the 2015-2017 LHC proton-proton collision data, 2019 JINST 14 P12006 [arXiv: 1908.00005] [INSPIRE].

[84] ATLAS collaboration, Electron efficiency measurements with the ATLAS detector using 2012 LHC proton-proton collision data, Eur. Phys. J. C 77 (2017) 195 [arXiv:1612.01456] [INSPIRE].

[85] ATLAS collaboration, Muon reconstruction performance of the ATLAS detector in proton-proton collision data at $\sqrt{s}=13$ TeV, Eur. Phys. J. C 76 (2016) 292 [arXiv: 1603.05598] [INSPIRE].

[86] ATLAS collaboration, Muon reconstruction performance in early $\sqrt{s}=13 \mathrm{TeV}$ data, ATL-PHYS-PUB-2015-037 (2015).

[87] ATLAS collaboration, Performance of missing transverse momentum reconstruction with the ATLAS detector using proton-proton collisions at $\sqrt{s}=13$ TeV, Eur. Phys. J. C 78 (2018) 903 [arXiv: 1802.08168] [inSPIRE].

[88] ATLAS collaboration, $E_{T}^{\text {miss }}$ performance in the ATLAS detector using 2015-2016 LHC p-p collisions, ATLAS-CONF-2018-023 (2018).

[89] M. Baak, G.J. Besjes, D. Côte, A. Koutsman, J. Lorenz and D. Short, HistFitter software framework for statistical data analysis, Eur. Phys. J. C 75 (2015) 153 [arXiv:1410.1280] [INSPIRE].

[90] G. Cowan, K. Cranmer, E. Gross and O. Vitells, Asymptotic formulae for likelihood-based tests of new physics, Eur. Phys. J. C 71 (2011) 1554 [Erratum ibid. 73 (2013) 2501] [arXiv: 1007.1727] [INSPIRE].

[91] A.L. Read, Presentation of search results: The CLs technique, J. Phys. G 28 (2002) 2693 [INSPIRE].

[92] ATLAS collaboration, ATLAS computing acknowledgements, ATL-SOFT-PUB-2020-001 (2020). 


\section{The ATLAS collaboration}

G. Aad $^{102}$, B. Abbott ${ }^{128}$, D.C. Abbott ${ }^{103}$, A. Abed Abud ${ }^{36}$, K. Abeling ${ }^{53}$, D.K. Abhayasinghe ${ }^{94}$, S.H. Abidi ${ }^{166}$, O.S. AbouZeid ${ }^{40}$, N.L. Abraham ${ }^{155}$, H. Abramowicz ${ }^{160}$, H. Abreu ${ }^{159}$, Y. Abulaiti ${ }^{6}$, B.S. Acharya ${ }^{67 a, 67 b, n}$, B. Achkar ${ }^{53}$, L. Adam ${ }^{100}$, C. Adam Bourdarios ${ }^{5}$, L. Adamczyk ${ }^{84 a}$, L. Adamek ${ }^{166}$, J. Adelman ${ }^{121}$, M. Adersberger ${ }^{114}$, A. Adiguzel ${ }^{12 c}$, S. Adorni ${ }^{54}$, T. Adye ${ }^{143}$,

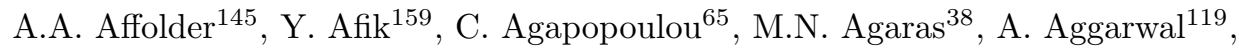
C. Agheorghiesei ${ }^{27 c}$, J.A. Aguilar-Saavedra ${ }^{139 f, 139 a, a d}$, A. Ahmad ${ }^{36}$, F. Ahmadov ${ }^{80}$, W.S. Ahmed ${ }^{104}$, X. Ai ${ }^{18}$, G. Aielli ${ }^{74 a}, 74 b$, S. Akatsuka ${ }^{86}$, T.P.A. Åkesson ${ }^{97}$, E. Akilli ${ }^{54}$, A.V. Akimov ${ }^{111}$, K. Al Khoury ${ }^{65}$, G.L. Alberghi ${ }^{23 b, 23 a}$, J. Albert ${ }^{175}$, M.J. Alconada Verzini ${ }^{160}$, S. Alderweireldt ${ }^{36}$, M. Aleksa ${ }^{36}$, I.N. Aleksandrov ${ }^{80}$, C. Alexa ${ }^{27 b}$, T. Alexopoulos ${ }^{10}$, A. Alfonsi $^{120}$, F. Alfonsi ${ }^{23 b, 23 a}$, M. Alhroob ${ }^{128}$, B. Ali ${ }^{141}$, S. Ali ${ }^{157}$, M. Aliev ${ }^{165}$, G. Alimonti ${ }^{69 a}$, C. Allaire ${ }^{36}$, B.M.M. Allbrooke ${ }^{155}$, B.W. Allen ${ }^{131}$, P.P. Allport ${ }^{21}$, A. Aloisio ${ }^{70 a}, 70 b$, F. Alonso ${ }^{89}$, C. Alpigiani ${ }^{147}$, E. Alunno Camelia ${ }^{74 a}, 74 b$, M. Alvarez Estevez ${ }^{99}$, M.G. Alviggi ${ }^{70 a}, 70 b$, Y. Amaral Coutinho ${ }^{81 b}$, A. Ambler ${ }^{104}$, L. Ambroz ${ }^{134}$, C. Amelung ${ }^{26}$, D. Amidei ${ }^{106}$, S.P. Amor Dos Santos ${ }^{139 a}$, S. Amoroso ${ }^{46}$, C.S. Amrouche ${ }^{54}$, F. An ${ }^{79}$, C. Anastopoulos ${ }^{148}$, N. Andari ${ }^{144}$, T. Andeen ${ }^{11}$, J.K. Anders ${ }^{20}$, S.Y. Andrean ${ }^{45 a, 45 b}$, A. Andreazza ${ }^{69 a, 69 b}$, V. Andrei ${ }^{61 a}$, C.R. Anelli ${ }^{175}$, S. Angelidakis ${ }^{9}$, A. Angerami ${ }^{39}$, A.V. Anisenkov ${ }^{122 b, 122 a}$, A. Annovi ${ }^{72 a}$, C. Antel ${ }^{54}$, M.T. Anthony ${ }^{148}$, E. Antipov ${ }^{129}$, M. Antonelli ${ }^{51}$, D.J.A. Antrim ${ }^{170}$, F. Anulli ${ }^{73 a}$, M. Aoki ${ }^{82}$, J.A. Aparisi Pozo ${ }^{173}$, M.A. Aparo ${ }^{155}$, L. Aperio Bella ${ }^{46}$,

N. Aranzabal Barrio ${ }^{36}$, V. Araujo Ferraz ${ }^{81 a}$, R. Araujo Pereira ${ }^{81 b}$, C. Arcangeletti ${ }^{51}$, A.T.H. Arce ${ }^{49}$, F.A. Arduh ${ }^{89}$, J-F. Arguin ${ }^{110}$, S. Argyropoulos ${ }^{52}$, J.-H. Arling 46 , A.J. Armbruster ${ }^{36}$, A. Armstrong ${ }^{170}$, O. Arnaez ${ }^{166}$, H. Arnold ${ }^{120}$, Z.P. Arrubarrena Tame ${ }^{114}$, G. Artoni ${ }^{134}$, S. Asai ${ }^{162}$, T. Asawatavonvanich ${ }^{164}$, N. Asbah ${ }^{59}$, E.M. Asimakopoulou ${ }^{171}$, L. Asquith ${ }^{155}$, J. Assahsah ${ }^{35 d}$, K. Assamagan ${ }^{29}$, R. Astalos ${ }^{28 a}$, R.J. Atkin ${ }^{33 a}$, M. Atkinson ${ }^{172}$, N.B. Atlay ${ }^{19}$, H. Atmani ${ }^{65}$, K. Augsten ${ }^{141}$, V.A. Austrup ${ }^{181}$, G. Avolio ${ }^{36}$, M.K. Ayoub ${ }^{15 a}$, G. Azuelos ${ }^{110, a l}$, H. Bachacou ${ }^{144}$, K. Bachas ${ }^{161}$, M. Backes ${ }^{134}$, F. Backman ${ }^{45 a, 45 b}$, P. Bagnaia ${ }^{73 a}, 73 b$, M. Bahmani ${ }^{85}$, H. Bahrasemani ${ }^{151}$, A.J. Bailey ${ }^{173}$, V.R. Bailey ${ }^{172}$, J.T. Baines ${ }^{143}$, C. Bakalis ${ }^{10}$, O.K. Baker ${ }^{182}$, P.J. Bakker ${ }^{120}$, E. Bakos ${ }^{16}$, D. Bakshi Gupta ${ }^{8}$, S. Balaji ${ }^{156}$, E.M. Baldin ${ }^{122 b, 122 a}$, P. Balek ${ }^{179}$, F. Balli ${ }^{144}$, W.K. Balunas ${ }^{134}$, J. Balz ${ }^{100}$, E. Banas ${ }^{85}$, M. Bandieramonte ${ }^{138}$, A. Bandyopadhyay ${ }^{24}$, Sw. Banerjee ${ }^{180, i}$, L. Barak ${ }^{160}$, W.M. Barbe ${ }^{38}$, E.L. Barberio ${ }^{105}$, D. Barberis ${ }^{55 b, 55 a}$, M. Barbero ${ }^{102}$, G. Barbour ${ }^{95}$, T. Barillari ${ }^{115}$, M-S. Barisits ${ }^{36}$, J. Barkeloo ${ }^{131}$, T. Barklow ${ }^{152}$, R. Barnea ${ }^{159}$, B.M. Barnett ${ }^{143}$, R.M. Barnett ${ }^{18}$, Z. Barnovska-Blenessy ${ }^{60 a}$, A. Baroncelli60a, G. Barone ${ }^{29}$, A.J. Barr ${ }^{134}$,

L. Barranco Navarro ${ }^{45 a, 45 b}$, F. Barreiro ${ }^{99}$, J. Barreiro Guimarães da Costa ${ }^{15 a}$, U. Barron ${ }^{160}$, S. Barsov ${ }^{137}$, F. Bartels ${ }^{61 a}$, R. Bartoldus ${ }^{152}$, G. Bartolini ${ }^{102}$, A.E. Barton ${ }^{90}$, P. Bartos ${ }^{28 a}$,

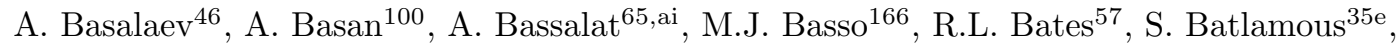
J.R. Batley ${ }^{32}$, B. Batool ${ }^{150}$, M. Battaglia ${ }^{145}$, M. Bauce ${ }^{73 a, 73 b}$, F. Bauer ${ }^{144}$, K.T. Bauer ${ }^{170}$, H.S. Bawa ${ }^{31}$, J.B. Beacham ${ }^{49}$, T. Beau ${ }^{135}$, P.H. Beauchemin ${ }^{169}$, F. Becherer ${ }^{52}$, P. Bechtle ${ }^{24}$, H.C. Beck ${ }^{53}$, H.P. Beck ${ }^{20, p}$, K. Becker ${ }^{177}$, C. Becot ${ }^{46}$, A. Beddall ${ }^{12 d}$, A.J. Beddall ${ }^{12 a}$, V.A. Bednyakov ${ }^{80}$, M. Bedognetti ${ }^{120}$, C.P. Bee ${ }^{154}$, T.A. Beermann ${ }^{181}$, M. Begalli ${ }^{81 b}$, M. Begel ${ }^{29}$, A. Behera ${ }^{154}$, J.K. Behr ${ }^{46}$, F. Beisiegel ${ }^{24}$, M. Belfkir ${ }^{5}$, A.S. Bell ${ }^{95}$, G. Bella ${ }^{160}$, L. Bellagamba ${ }^{23 b}$,

A. Bellerive ${ }^{34}$, P. Bellos ${ }^{9}$, K. Beloborodov ${ }^{122 b, 122 a}$, K. Belotskiy ${ }^{112}$, N.L. Belyaev ${ }^{112}$, D. Benchekroun ${ }^{35 a}$, N. Benekos ${ }^{10}$, Y. Benhammou ${ }^{160}$, D.P. Benjamin ${ }^{6}$, M. Benoit ${ }^{54}$, J.R. Bensinger ${ }^{26}$, S. Bentvelsen ${ }^{120}$, L. Beresford ${ }^{134}$, M. Beretta ${ }^{51}$, D. Berge ${ }^{19}$,

E. Bergeaas Kuutmann ${ }^{171}$, N. Berger ${ }^{5}$, B. Bergmann ${ }^{141}$, L.J. Bergsten ${ }^{26}$, J. Beringer ${ }^{18}$, S. Berlendis ${ }^{7}$, G. Bernardi ${ }^{135}$, C. Bernius ${ }^{152}$, F.U. Bernlochner ${ }^{24}$, T. Berry ${ }^{94}$, P. Berta ${ }^{100}$, C. Bertella ${ }^{15 a}$, A. Berthold ${ }^{48}$, I.A. Bertram ${ }^{90}$, O. Bessidskaia Bylund ${ }^{181}$, N. Besson ${ }^{144}$, 
A. Bethani ${ }^{101}$, S. Bethke ${ }^{115}$, A. Betti ${ }^{42}$, A.J. Bevan ${ }^{93}$, J. Beyer ${ }^{115}$, D.S. Bhattacharya ${ }^{176}$, P. Bhattarai ${ }^{26}$, V.S. Bhopatkar ${ }^{6}$, R. Bi $^{138}$, R.M. Bianchi ${ }^{138}$, O. Biebel ${ }^{114}$, D. Biedermann ${ }^{19}$, R. Bielski ${ }^{36}$, K. Bierwagen ${ }^{100}$, N.V. Biesuz ${ }^{72 a, 72 b}$, M. Biglietti ${ }^{75 a}$, T.R.V. Billoud ${ }^{110}$, M. Bindi $^{53}$, A. Bingul ${ }^{12 \mathrm{~d}}$, C. Bini ${ }^{73 a, 73 b}$, S. Biondi ${ }^{23 b}$,23a , C.J. Birch-sykes ${ }^{101}$, M. Birman ${ }^{179}$, T. Bisanz ${ }^{53}$, J.P. Biswal ${ }^{3}$, D. Biswas ${ }^{180, \mathrm{i}}$, A. Bitadze ${ }^{101}$, C. Bittrich ${ }^{48}$, K. Bjørke ${ }^{133}$, T. Blazek ${ }^{28 a}$, I. Bloch ${ }^{46}$, C. Blocker ${ }^{26}$, A. Blue ${ }^{57}$, U. Blumenschein ${ }^{93}$, G.J. Bobbink ${ }^{120}$, V.S. Bobrovnikov ${ }^{122 b, 122 a}$, S.S. Bocchetta ${ }^{97}$, D. Boerner ${ }^{46}$, D. Bogavac ${ }^{14}$, A.G. Bogdanchikov ${ }^{122 b, 122 a}$, C. Bohm ${ }^{45 a}$, V. Boisvert ${ }^{94}$, P. Bokan ${ }^{53,171,53}$, T. Bold ${ }^{84 a}$, A.E. Bolz ${ }^{61 b}$, M. Bomben ${ }^{135}$, M. Bona ${ }^{93}$, J.S. Bonilla ${ }^{131}$, M. Boonekamp ${ }^{144}$, C.D. Booth ${ }^{94}$, H.M. Borecka-Bielska ${ }^{91}$, L.S. Borgna ${ }^{95}$, A. Borisov ${ }^{123}$, G. Borissov ${ }^{90}$, J. Bortfeldt ${ }^{36}$, D. Bortoletto ${ }^{134}$, D. Boscherini ${ }^{23 b}$, M. Bosman ${ }^{14}$, J.D. Bossio Sola ${ }^{104}$, K. Bouaouda ${ }^{35 a}$, J. Boudreau ${ }^{138}$, E.V. Bouhova-Thacker ${ }^{90}$, D. Boumediene ${ }^{38}$, S.K. Boutle ${ }^{57}$, A. Boveia ${ }^{127}$, J. Boyd ${ }^{36}$, D. Boye ${ }^{33 c}$, I.R. Boyko ${ }^{80}$, A.J. Bozson ${ }^{94}$, J. Bracinik ${ }^{21}$, N. Brahimi ${ }^{60 d}$, G. Brandt ${ }^{181}$, O. Brandt ${ }^{32}$, F. Braren ${ }^{46}$, B. Brau ${ }^{103}$, J.E. Brau ${ }^{131}$, W.D. Breaden Madden ${ }^{57}$, K. Brendlinger ${ }^{46}$, L. Brenner ${ }^{46}$, R. Brenner ${ }^{171}$, S. Bressler ${ }^{179}$, B. Brickwedde ${ }^{100}$, D.L. Briglin ${ }^{21}$, D. Britton ${ }^{57}$, D. Britzger ${ }^{115}$, I. Brock ${ }^{24}$, R. Brock ${ }^{107}$, G. Brooijmans ${ }^{39}$, W.K. Brooks ${ }^{146 \mathrm{~d}}$, E. Brost ${ }^{29}$, P.A. Bruckman de Renstrom ${ }^{85}$, B. Brüers ${ }^{46}$,

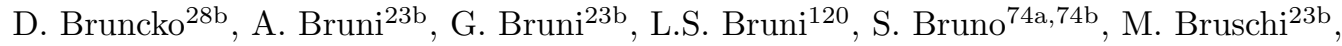
N. Bruscino ${ }^{73 a, 73 b}$, L. Bryngemark ${ }^{152}$, T. Buanes ${ }^{17}$, Q. Buat ${ }^{36}$, P. Buchholz ${ }^{150}$, A.G. Buckley ${ }^{57}$, I.A. Budagov ${ }^{80}$, M.K. Bugge ${ }^{133}$, F. Bührer ${ }^{52}$, O. Bulekov ${ }^{112}$, B.A. Bullard ${ }^{59}$, T.J. Burch ${ }^{121}$, S. Burdin ${ }^{91}$, C.D. Burgard ${ }^{120}$, A.M. Burger ${ }^{129}$, B. Burghgrave ${ }^{8}$, J.T.P. Burr ${ }^{46}$, C.D. Burton ${ }^{11}$, J.C. Burzynski ${ }^{103}$, V. Büscher ${ }^{100}$, E. Buschmann ${ }^{53}$, P.J. Bussey ${ }^{57}$, J.M. Butler ${ }^{25}$, C.M. Buttar ${ }^{57}$, J.M. Butterworth ${ }^{95}$, P. Butti ${ }^{36}$, W. Buttinger ${ }^{36}$, C.J. Buxo Vazquez ${ }^{107}$, A. Buzatu ${ }^{157}$, A.R. Buzykaev ${ }^{122 b, 122 a}$, G. Cabras ${ }^{23 b, 23 a}$, S. Cabrera Urbán ${ }^{173}$, D. Caforio ${ }^{56}$, H. Cai ${ }^{138}$, V.M.M. Cairo ${ }^{152}$, O. Cakir ${ }^{4 a}$, N. Calace $^{36}$, P. Calafiura ${ }^{18}$, G. Calderini ${ }^{135}$, P. Calfayan ${ }^{66}$, G. Callea ${ }^{57}$, L.P. Caloba ${ }^{81 b}$, A. Caltabiano ${ }^{74 a, 74 b}$, S. Calvente Lopez ${ }^{99}$, D. Calvet ${ }^{38}$, S. Calvet ${ }^{38}$, T.P. Calvet ${ }^{102}$, M. Calvetti ${ }^{72 a, 72 b}$, R. Camacho Toro ${ }^{135}$, S. Camarda ${ }^{36}$, D. Camarero Munoz ${ }^{99}$, P. Camarri ${ }^{74 a, 74 b}$, M.T. Camerlingo ${ }^{75 a, 75 b}$, D. Cameron ${ }^{133}$, C. Camincher ${ }^{36}$, S. Campana ${ }^{36}$,

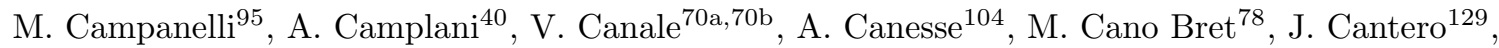
T. $\mathrm{Cao}^{160}$, Y. Cao ${ }^{172}$, M.D.M. Capeans Garrido ${ }^{36}$, M. Capua ${ }^{41 \mathrm{~b}, 41 \mathrm{a}}$, R. Cardarelli ${ }^{74 a}$,

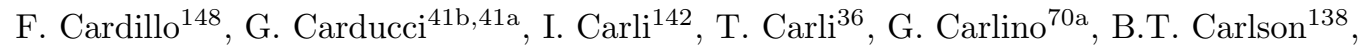
E.M. Carlson ${ }^{175,167 a}$, L. Carminati ${ }^{69 a, 69 b}$, R.M.D. Carney ${ }^{152}$, S. Caron ${ }^{119}$, E. Carquin ${ }^{146 d}$, S. Carrá ${ }^{46}$, G. Carratta ${ }^{23 b, 23 a}$, J.W.S. Carter ${ }^{166}$, T.M. Carter ${ }^{50}$, M.P. Casado ${ }^{14, \mathrm{f}}$, A.F. Casha ${ }^{166}$, F.L. Castillo ${ }^{173}$, L. Castillo Garcia ${ }^{14}$, V. Castillo Gimenez ${ }^{173}$, N.F. Castro ${ }^{139 a, 139 e}$,

A. Catinaccio ${ }^{36}$, J.R. Catmore ${ }^{133}$, A. Cattai ${ }^{36}$, V. Cavaliere ${ }^{29}$, E. Cavallaro ${ }^{14}$, V. Cavasinni ${ }^{72 a, 72 b}$, E. Celebi ${ }^{12 b}$, F. Celli ${ }^{134}$, K. Cerny ${ }^{130}$, A.S. Cerqueira ${ }^{81 a}$, A. Cerri ${ }^{155}$, L. Cerrito ${ }^{74 a, 74 b}$, F. Cerutti ${ }^{18}$, A. Cervelli ${ }^{23 b, 23 a}$, S.A. Cetin ${ }^{12 b}$, Z. Chadi ${ }^{35 a}$, D. Chakraborty ${ }^{121}$, J. Chan ${ }^{180}$, W.S. Chan ${ }^{120}$, W.Y. Chan ${ }^{91}$, J.D. Chapman ${ }^{32}$, B. Chargeishvili ${ }^{158 b}$, D.G. Charlton ${ }^{21}$, T.P. Charman ${ }^{93}$, C.C. $\mathrm{Chau}^{34}$, S. $\mathrm{Che}^{127}$, S. Chekanov $^{6}$, S.V. Chekulaev ${ }^{167 a}$, G.A. Chelkov ${ }^{80, a g}$, B. Chen ${ }^{79}$, C. Chen ${ }^{60 a}$, C.H. Chen ${ }^{79}$, H. Chen ${ }^{29}$, J. Chen ${ }^{60 a}$, J. Chen ${ }^{39}$, J. Chen ${ }^{26}$, S. Chen ${ }^{136}$, S.J. Chen ${ }^{15 c}$, X. Chen ${ }^{15 b}$, Y. Chen ${ }^{60 a}$, Y-H. Chen ${ }^{46}$, H.C. Cheng ${ }^{63 a}$, H.J. Cheng ${ }^{15 a}$, A. Cheplakov ${ }^{80}$, E. Cheremushkina ${ }^{123}$, R. Cherkaoui El Moursli ${ }^{35 e}$, E. Cheu ${ }^{7}$, K. Cheung ${ }^{64}$, T.J.A. Chevalérias ${ }^{144}$, L. Chevalier ${ }^{144}$, V. Chiarella ${ }^{51}$, G. Chiarelli ${ }^{72 a}$, G. Chiodini ${ }^{68 a}$, A.S. Chisholm ${ }^{21}$, A. Chitann ${ }^{27 b}$, I. Chiu ${ }^{162}$, Y.H. Chiu ${ }^{175}$, M.V. Chizhov ${ }^{80}$, K. Choi ${ }^{11}$, A.R. Chomont ${ }^{73 a, 73 b}$, S. Chouridou ${ }^{161}$, Y.S. Chow ${ }^{120}$, L.D. Christopher ${ }^{33 e}$, M.C. Chu ${ }^{63 a}$, X. Chu ${ }^{15 a, 15 d}$, J. Chudoba ${ }^{140}$, J.J. Chwastowski ${ }^{85}$, L. Chytka ${ }^{130}$, D. Cieri ${ }^{115}$, K.M. Ciesla ${ }^{85}$,

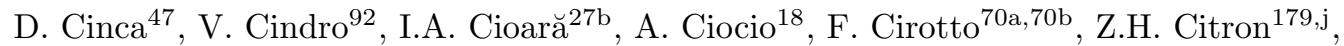
M. Citterio ${ }^{69 a}$, D.A. Ciubotaru² ${ }^{27 b}$, B.M. Ciungu ${ }^{166}$, A. Clark ${ }^{54}$, M.R. Clark ${ }^{39}$, P.J. Clark ${ }^{50}$, S.E. Clawson ${ }^{101}$, C. Clement ${ }^{45 a, 45 b}$, Y. Coadou ${ }^{102}$, M. Cobal ${ }^{67 a, 67 c}$, A. Coccaro ${ }^{55 b}$, J. Cochran ${ }^{79}$, 
R. Coelho Lopes De $\mathrm{Sa}^{103}$, H. Cohen ${ }^{160}$, A.E.C. Coimbra ${ }^{36}$, B. Cole ${ }^{39}$, A.P. Colijn ${ }^{120}$, J. Collot ${ }^{58}$, P. Conde Muiño ${ }^{139 a, 139 h}$, S.H. Connell ${ }^{33 c}$, I.A. Connelly ${ }^{57}$, S. Constantinescu ${ }^{27 b}$,

F. Conventi $70 a, a m$, A.M. Cooper-Sarkar ${ }^{134}$, F. Cormier ${ }^{174}$, K.J.R. Cormier ${ }^{166}$, L.D. Corpe ${ }^{95}$,

M. Corradi ${ }^{73 a}, 73 b$, E.E. Corrigan ${ }^{97}$, F. Corriveau ${ }^{104, a b}$, M.J. Costa ${ }^{173}$, F. Costanza ${ }^{5}$,

D. Costanzo ${ }^{148}$, G. Cowan ${ }^{94}$, J.W. Cowley ${ }^{32}$, J. Crane ${ }^{101}$, K. Cranmer ${ }^{125}$, R.A. Creager ${ }^{136}$,

S. Crépé-Renaudin ${ }^{58}$, F. Crescioli ${ }^{135}$, M. Cristinziani ${ }^{24}$, V. Croft $^{169}$, G. Crosetti $^{41 b, 41 a}$, A. Cueto ${ }^{5}$,

T. Cuhadar Donszelmann ${ }^{170}$, H. Cui ${ }^{15 a, 15 d}$, A.R. Cukierman ${ }^{152}$, W.R. Cunningham ${ }^{57}$,

S. Czekierda ${ }^{85}$, P. Czodrowski ${ }^{36}$, M.M. Czurylo ${ }^{61 b}$, M.J. Da Cunha Sargedas De Sousa ${ }^{60 b}$, J.V. Da Fonseca Pinto ${ }^{81 b}$, C. Da Via ${ }^{101}$, W. Dabrowski ${ }^{84 a}$, F. Dachs ${ }^{36}$, T. Dado ${ }^{28 a}$, S. Dahbi ${ }^{33 e}$, T. Dai ${ }^{106}$, C. Dallapiccola ${ }^{103}$, M. Dam ${ }^{40}$, G. D'amen ${ }^{29}$, V. D'Amico ${ }^{75 a, 75 b}$, J. Damp ${ }^{100}$, J.R. Dandoy ${ }^{136}$, M.F. Daneri ${ }^{30}$, M. Danninger ${ }^{151}$, V. Dao ${ }^{36}$, G. Darbo ${ }^{55 b}$, O. Dartsi ${ }^{5}$, A. Dattagupta ${ }^{131}$, T. Daubney ${ }^{46}$, S. D’Auria ${ }^{69 a, 69 b}$, C. David ${ }^{167 b}$, T. Davidek ${ }^{142}$, D.R. Davis ${ }^{49}$, I. Dawson ${ }^{148}$, K. De ${ }^{8}$, R. De Asmundis ${ }^{70 a}$, M. De Beurs ${ }^{120}$, S. De Castro ${ }^{23 b, 23 a}$, N. De Groot ${ }^{119}$, P. de Jong ${ }^{120}$, H. De la Torre ${ }^{107}$, A. De Maria ${ }^{15 c}$, D. De Pedis ${ }^{73 a}$, A. De Salvo ${ }^{73 a}$, U. De Sanctis ${ }^{74 a, 74 b}$, M. De Santis ${ }^{74 a, 74 b}$, A. De Santo ${ }^{155}$, J.B. De Vivie De Regie ${ }^{65}$, C. Debenedetti ${ }^{145}$, D.V. Dedovich ${ }^{80}$, A.M. Deiana ${ }^{42}$, J. Del Peso ${ }^{99}$, Y. Delabat Diaz ${ }^{46}$, D. Delgove ${ }^{65}$, F. Deliot ${ }^{144}$, C.M. Delitzsch ${ }^{7}$, M. Della Pietra ${ }^{70 a, 70 b}$, D. Della Volpe ${ }^{54}$,

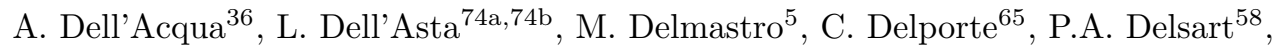
D.A. DeMarco ${ }^{166}$, S. Demers ${ }^{182}$, M. Demichev ${ }^{80}$, G. Demontigny ${ }^{110}$, S.P. Denisov ${ }^{123}$,

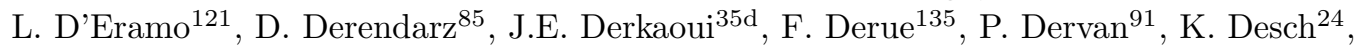
K. Dette ${ }^{166}$, C. Deutsch ${ }^{24}$, M.R. Devesa ${ }^{30}$, P.O. Deviveiros ${ }^{36}$, F.A. Di Bello ${ }^{73 a}, 73 b$, A. Di Ciaccio ${ }^{74 a, 74 b}$, L. Di Ciaccio5 5 , W.K. Di Clemente ${ }^{136}$, C. Di Donato ${ }^{70 a, 70 b}$, A. Di Girolamo ${ }^{36}$, G. Di Gregorio ${ }^{72 a, 72 b}$, B. Di Micco ${ }^{75 a, 75 b}$, R. Di Nardo ${ }^{75 a, 75 b}$, K.F. Di Petrillo ${ }^{59}$, R. Di Sipio ${ }^{166}$, C. Diaconu ${ }^{102}$, F.A. Dias ${ }^{40}$, T. Dias Do Vale ${ }^{139 a}$, M.A. Diaz ${ }^{146 a}$, F.G. Diaz Capriles ${ }^{24}$, J. Dickinson ${ }^{18}$, E.B. Diehl ${ }^{106}$, J. Dietrich ${ }^{19}$, S. Díez Cornell ${ }^{46}$, A. Dimitrievska ${ }^{18}$, W. Ding ${ }^{15 b}$, J. Dingfelder ${ }^{24}$, S.J. Dittmeier ${ }^{61 b}$, F. Dittus ${ }^{36}$, F. Djama ${ }^{102}$, T. Djobava ${ }^{158 b}$, J.I. Djuvsland ${ }^{17}$, M.A.B. Do Vale ${ }^{81 c}$, M. Dobre ${ }^{27 b}$, D. Dodsworth ${ }^{26}$, C. Doglioni ${ }^{97}$, J. Dolejsi ${ }^{142}$, Z. Dolezal ${ }^{142}$,

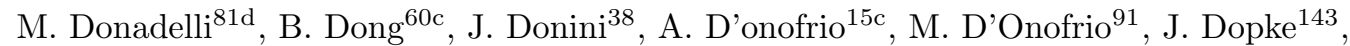

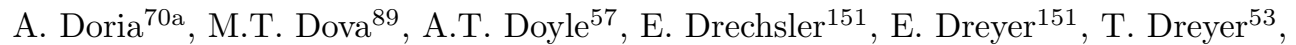
A.S. Drobac ${ }^{169}$, D. Du ${ }^{60 b}$, T.A. du Pree ${ }^{120}$, Y. Duan ${ }^{60 d}$, F. Dubinin ${ }^{111}$, M. Dubovsky ${ }^{28 a}$, A. Dubreuil ${ }^{54}$, E. Duchovni ${ }^{179}$, G. Duckeck ${ }^{114}$, O.A. Ducu ${ }^{27 b}$, D. Duda ${ }^{115}$, A. Dudarev ${ }^{36}$, A.C. Dudder ${ }^{100}$, E.M. Duffield ${ }^{18}$, M. D’uffizi ${ }^{101}$, L. Duflot ${ }^{65}$, M. Dührssen ${ }^{36}$, C. Dülsen ${ }^{181}$,

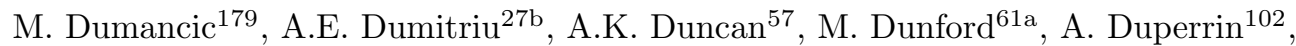
H. Duran Yildiz ${ }^{4 a}$, M. Düren ${ }^{56}$, A. Durglishvili ${ }^{158 b}$, D. Duschinger ${ }^{48}$, B. Dutta ${ }^{46}$, D. Duvnjak ${ }^{1}$, G.I. Dyckes ${ }^{136}$, M. Dyndal ${ }^{36}$, S. Dysch ${ }^{101}$, B.S. Dziedzic ${ }^{85}$, M.G. Eggleston ${ }^{49}$, T. Eifert ${ }^{8}$, G. Eigen ${ }^{17}$, K. Einsweiler ${ }^{18}$, T. Ekelof ${ }^{171}$, H. El Jarrari ${ }^{35 e}$, V. Ellajosyula ${ }^{171}$, M. Ellert ${ }^{171}$, F. Ellinghaus ${ }^{181}$, A.A. Elliot ${ }^{93}$, N. Ellis ${ }^{36}$, J. Elmsheuser ${ }^{29}$, M. Elsing ${ }^{36}$, D. Emeliyanov ${ }^{143}$, A. Emerman ${ }^{39}$, Y. Enari ${ }^{162}$, M.B. Epland ${ }^{49}$, J. Erdmann ${ }^{47}$, A. Ereditato ${ }^{20}$, P.A. Erland ${ }^{85}$, M. Errenst ${ }^{36}$, M. Escalier ${ }^{65}$, C. Escobar ${ }^{173}$, O. Estrada Pastor ${ }^{173}$, E. Etzion ${ }^{160}$, H. Evans ${ }^{66}$, M.O. Evans ${ }^{155}$, A. Ezhilov ${ }^{137}$, F. Fabbri ${ }^{57}$, L. Fabbri ${ }^{23 b, 23 a}$, V. Fabiani ${ }^{119}$, G. Facini ${ }^{177}$, R.M. Faisca Rodrigues Pereira ${ }^{139 a}$, R.M. Fakhrutdinov ${ }^{123}$, S. Falciano ${ }^{73 a}$, P.J. Falke ${ }^{24}$, S. Falke ${ }^{36}$, J. Faltova ${ }^{142}$, Y. Fang ${ }^{15 a}$, Y. Fang ${ }^{15 a}$, G. Fanourakis ${ }^{44}$, M. Fanti ${ }^{69 a, 69 b}$, M. Faraj67a,67c,, ,

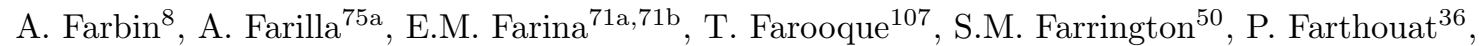
F. Fassi ${ }^{35 e}$, P. Fassnacht ${ }^{36}$, D. Fassouliotis ${ }^{9}$, M. Faucci Giannelli ${ }^{50}$, W.J. Fawcett ${ }^{32}$, L. Fayard ${ }^{65}$, O.L. Fedin ${ }^{137, o}$, W. Fedorko ${ }^{174}$, A. Fehr ${ }^{20}$, M. Feickert ${ }^{172}$, L. Feligioni ${ }^{102}$, A. Fell ${ }^{148}$, C. Feng ${ }^{60 b}$, M. Feng ${ }^{49}$, M.J. Fenton ${ }^{170}$, A.B. Fenyuk ${ }^{123}$, S.W. Ferguson ${ }^{43}$, J. Ferrando ${ }^{46}$, A. Ferrante ${ }^{172}$, A. Ferrari ${ }^{171}$, P. Ferrari ${ }^{120}$, R. Ferrari ${ }^{71 a}$, D.E. Ferreira de Lima ${ }^{61 b}$, A. Ferrer ${ }^{173}$, D. Ferrere ${ }^{54}$, C. Ferretti ${ }^{106}$, F. Fiedler ${ }^{100}$, A. Filipčič ${ }^{92}$, F. Filthaut ${ }^{119}$, K.D. Finelli ${ }^{25}$, 
M.C.N. Fiolhais ${ }^{139 a, 139 c, a}$, L. Fiorini ${ }^{173}$, F. Fischer ${ }^{114}$, J. Fischer ${ }^{100}$, W.C. Fisher ${ }^{107}$, T. Fitschen ${ }^{21}$, I. Fleck ${ }^{150}$, P. Fleischmann ${ }^{106}$, T. Flick ${ }^{181}$, B.M. Flierl ${ }^{114}$, L. Flores ${ }^{136}$, L.R. Flores Castillo ${ }^{63 a}$, F.M. Follega ${ }^{76 a, 76 b}$, N. Fomin ${ }^{17}$, J.H. Foo ${ }^{166}$, G.T. Forcolin ${ }^{76 a, 76 b}$, B.C. Forland ${ }^{66}$, A. Formica ${ }^{144}$, F.A. Förster ${ }^{14}$, A.C. Forti ${ }^{101}$, E. Fortin ${ }^{102}$, M.G. Foti ${ }^{134}$, D. Fournier ${ }^{65}$, H. Fox ${ }^{90}$, P. Francavilla ${ }^{72 a, 72 b}$, S. Francescato ${ }^{73 a, 73 b}$, M. Franchini ${ }^{23 b, 23 a}$, S. Franchino ${ }^{61 a}$, D. Francis ${ }^{36}$, L. Franco ${ }^{5}$, L. Franconi ${ }^{20}$, M. Franklin ${ }^{59}$, G. Frattari ${ }^{73 a, 73 b}$, A.N. Fray ${ }^{93}$, P.M. Freeman ${ }^{21}$, B. Freund ${ }^{110}$, W.S. Freund ${ }^{81 b}$, E.M. Freundlich ${ }^{47}$, D.C. Frizzell ${ }^{128}$, D. Froidevaux ${ }^{36}$, J.A. Frost ${ }^{134}$, M. Fujimoto ${ }^{126}$, C. Fukunaga ${ }^{163}$, E. Fullana Torregrosa ${ }^{173}$, T. Fusayasu ${ }^{116}$, J. Fuster ${ }^{173}$, A. Gabrielli ${ }^{23 b, 23 a}$, A. Gabrielli ${ }^{36}$, S. Gadatsch ${ }^{54}$, P. Gadow ${ }^{115}$, G. Gagliardi ${ }^{55 b, 55 a}$, L.G. Gagnon ${ }^{110}$, G.E. Gallardo ${ }^{134}$, E.J. Gallas ${ }^{134}$, B.J. Gallop ${ }^{143}$, G. Galster ${ }^{40}$, R. Gamboa Goni ${ }^{93}$, K.K. Gan ${ }^{127}$, S. Ganguly ${ }^{179}$, J. Gao ${ }^{60 a}$, Y. Gao ${ }^{50}$, Y.S. Gao ${ }^{31,1}$, F.M. Garay Walls ${ }^{146 a}$, C. García ${ }^{173}$, J.E. García Navarro ${ }^{173}$, J.A. García Pascual ${ }^{15 a}$, C. Garcia-Argos ${ }^{52}$, M. Garcia-Sciveres ${ }^{18}$, R.W. Gardner ${ }^{37}$, N. Garelli ${ }^{152}$, S. Gargiulo ${ }^{52}$, C.A. Garner ${ }^{166}$, V. Garonne ${ }^{133}$, S.J. Gasiorowski ${ }^{147}$, P. Gaspar ${ }^{81 b}$, A. Gaudiello ${ }^{55 b, 55 a}$, G. Gaudio ${ }^{71 a}$, I.L. Gavrilenko ${ }^{111}$, A. Gavrilyuk ${ }^{124}$, C. Gay ${ }^{174}$, G. Gaycken ${ }^{46}$, E.N. Gazis ${ }^{10}$, A.A. Geanta ${ }^{27 b}$, C.M. Gee ${ }^{145}$, C.N.P. Gee ${ }^{143}$, J. Geisen ${ }^{97}$, M. Geisen ${ }^{100}$, C. Gemme ${ }^{55 b}$, M.H. Genest ${ }^{58}$, C. Geng ${ }^{106}$, S. Gentile ${ }^{73 a, 73 b}$, S. George ${ }^{94}$, T. Geralis ${ }^{44}$, L.O. Gerlach ${ }^{53}$, P. Gessinger-Befurt ${ }^{100}$, G. Gessner ${ }^{47}$, S. Ghasemi ${ }^{150}$, M. Ghasemi Bostanabad ${ }^{175}$, M. Ghneimat ${ }^{150}$, A. Ghosh ${ }^{65}$, A. Ghosh ${ }^{78}$, B. Giacobbe ${ }^{23 b}$, S. Giagu ${ }^{73 a, 73 b}$, N. Giangiacomi ${ }^{23 b, 23 a}$, P. Giannetti ${ }^{72 a}$, A. Giannini ${ }^{70 a}, 70 b$, G. Giannini ${ }^{14}$, S.M. Gibson ${ }^{94}$, M. Gignac ${ }^{145}$, D.T. Gil ${ }^{84 b}$, D. Gillberg ${ }^{34}$, G. Gilles ${ }^{181}$, D.M. Gingrich ${ }^{3, a l}$, M.P. Giordani $67 a, 67 c$, P.F. Giraud ${ }^{144}$, G. Giugliarelli ${ }^{67 a, 67 c}$, D. Giugni69a ${ }^{69}$ F. Giuli ${ }^{74 a, 74 b}$, S. Gkaitatzis ${ }^{161}$, I. Gkialas ${ }^{9, g}$, E.L. Gkougkousis ${ }^{14}$, P. Gkountoumis ${ }^{10}$, L.K. Gladilin ${ }^{113}$, C. Glasman ${ }^{99}$, J. Glatzer ${ }^{14}$, P.C.F. Glaysher ${ }^{46}$, A. Glazov ${ }^{46}$, G.R. Gledhill ${ }^{131}$, I. Gnesi ${ }^{41 b, b}$, M. Goblirsch-Kolb ${ }^{26}$, D. Godin ${ }^{110}$, S. Goldfarb ${ }^{105}$, T. Golling ${ }^{54}$, D. Golubkov ${ }^{123}$, A. Gomes ${ }^{139 a, 139 b}$, R. Goncalves Gama ${ }^{53}$, R. Gonçalo ${ }^{139 a, 139 c}$, G. Gonella ${ }^{131}$, L. Gonella ${ }^{21}$, A. Gongadze ${ }^{80}$, F. Gonnella ${ }^{21}$, J.L. Gonski ${ }^{39}$, S. González de la $\mathrm{Hoz}^{173}$, S. Gonzalez Fernandez ${ }^{14}$, C. Gonzalez Renteria ${ }^{18}$, R. Gonzalez Suarez ${ }^{171}$, S. Gonzalez-Sevilla ${ }^{54}$, G.R. Gonzalvo Rodriguez ${ }^{173}$, L. Goossens ${ }^{36}$, N.A. Gorasia ${ }^{21}$, P.A. Gorbounov ${ }^{124}$, H.A. Gordon ${ }^{29}$, B. Gorini ${ }^{36}$, E. Gorini68a,68b , A. Gorišek ${ }^{92}$, A.T. Goshaw ${ }^{49}$, M.I. Gostkin ${ }^{80}$, C.A. Gottardo ${ }^{119}$, M. Gouighri ${ }^{35 b}$, A.G. Goussiou ${ }^{147}$, N. Govender ${ }^{33 c}$, C. Goy ${ }^{5}$, I. Grabowska-Bold ${ }^{84 a}$, E.C. Graham ${ }^{91}$, J. Gramling ${ }^{170}$, E. Gramstad ${ }^{133}$, S. Grancagnolo ${ }^{19}$, M. Grandi ${ }^{155}$, V. Gratchev ${ }^{137}$, P.M. Gravila ${ }^{27 f}$, F.G. Gravili68a,68b ${ }^{6}$ C. Gray ${ }^{57}$, H.M. Gray ${ }^{18}$, C. Grefe ${ }^{24}$, K. Gregersen ${ }^{97}$, I.M. Gregor ${ }^{46}$, P. Grenier ${ }^{152}$, K. Grevtsov ${ }^{46}$, C. Grieco ${ }^{14}$, N.A. Grieser ${ }^{128}$, A.A. Grillo ${ }^{145}$, K. Grimm ${ }^{31, \mathrm{k}}$, S. Grinstein ${ }^{14, \mathrm{w}}$, J.-F. Grivaz ${ }^{65}$, S. Groh ${ }^{100}$, E. Gross ${ }^{179}$, J. Grosse-Knetter ${ }^{53}$, Z.J. Grout ${ }^{95}$, C. Grud ${ }^{106}$, A. Grummer ${ }^{118}$, J.C. Grundy ${ }^{134}$, L. Guan ${ }^{106}$, W. Guan ${ }^{180}$, C. Gubbels ${ }^{174}$, J. Guenther ${ }^{36}$, A. Guerguichon ${ }^{65}$, J.G.R. Guerrero Rojas ${ }^{173}$, F. Guescini ${ }^{115}$, D. Guest ${ }^{170}$, R. Gugel ${ }^{100}$, T. Guillemin ${ }^{5}$, S. Guindon ${ }^{36}$,

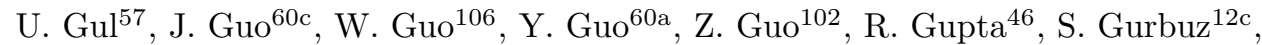
G. Gustavino ${ }^{128}$, M. Guth ${ }^{52}$, P. Gutierrez ${ }^{128}$, C. Gutschow ${ }^{95}$, C. Guyot ${ }^{144}$, C. Gwenlan ${ }^{134}$, C.B. Gwilliam ${ }^{91}$, E.S. Haaland ${ }^{133}$, A. $\operatorname{Haas}^{125}$, C. Haber ${ }^{18}$, H.K. Hadavand ${ }^{8}$, A. Hadef ${ }^{60 a}$, M. Haleem ${ }^{176}$, J. Haley ${ }^{129}$, J.J. Hall ${ }^{148}$, G. Halladjian ${ }^{107}$, G.D. Hallewell ${ }^{102}$, K. Hamano ${ }^{175}$,

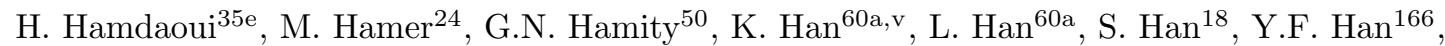
K. Hanagaki ${ }^{82, t}$, M. Hance ${ }^{145}$, D.M. Handl ${ }^{114}$, M.D. Hank ${ }^{37}$, R. Hankache ${ }^{135}$, E. Hansen ${ }^{97}$, J.B. Hansen ${ }^{40}$, J.D. Hansen ${ }^{40}$, M.C. Hansen ${ }^{24}$, P.H. Hansen ${ }^{40}$, E.C. Hanson ${ }^{101}$, K. Hara ${ }^{168}$, T. Harenberg ${ }^{181}$, S. Harkusha ${ }^{108}$, P.F. Harrison ${ }^{177}$, N.M. Hartman ${ }^{152}$, N.M. Hartmann ${ }^{114}$, Y. Hasegawa ${ }^{149}$, A. Hasib ${ }^{50}$, S. Hassani ${ }^{144}$, S. Haug ${ }^{20}$, R. Hauser ${ }^{107}$, L.B. Havener ${ }^{39}$, M. Havranek ${ }^{141}$, C.M. Hawkes ${ }^{21}$, R.J. Hawkings ${ }^{36}$, S. Hayashida ${ }^{117}$, D. Hayden ${ }^{107}$, C. Hayes ${ }^{106}$,

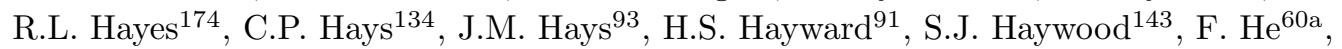


M.P. Heath ${ }^{50}$, V. Hedberg ${ }^{97}$, S. Heer ${ }^{24}$, A.L. Heggelund ${ }^{133}$, C. Heidegger ${ }^{52}$, K.K. Heidegger ${ }^{52}$, W.D. Heidorn ${ }^{79}$, J. Heilman ${ }^{34}$, S. Heim ${ }^{46}$, T. Heim ${ }^{18}$, B. Heinemann ${ }^{46, a j}$, J.G. Heinlein ${ }^{136}$, J.J. Heinrich ${ }^{131}$, L. Heinrich ${ }^{36}$, J. Hejbal ${ }^{140}$, L. Helary ${ }^{61 b}$, A. Held ${ }^{125}$, S. Hellesund ${ }^{133}$, C.M. Helling ${ }^{145}$, S. Hellman ${ }^{45 a, 45 b}$, C. Helsens ${ }^{36}$, R.C.W. Henderson ${ }^{90}$, Y. Heng ${ }^{180}$, L. Henkelmann ${ }^{32}$, A.M. Henriques Correia ${ }^{36}$, H. Herde ${ }^{26}$, Y. Hernández Jiménez ${ }^{33 e}, H . H^{2}{ }^{100}$, M.G. Herrmann ${ }^{114}$, T. Herrmann ${ }^{48}$, G. Herten ${ }^{52}$, R. Hertenberger ${ }^{114}$, L. Hervas ${ }^{36}$, T.C. Herwig ${ }^{136}$, G.G. Hesketh ${ }^{95}$, N.P. Hessey ${ }^{167 a}$, H. Hibi ${ }^{83}$, A. Higashida ${ }^{162}$, S. Higashino ${ }^{82}$, E. Higón-Rodriguez ${ }^{173}$, K. Hildebrand ${ }^{37}$, J.C. Hill ${ }^{32}$, K.K. Hill ${ }^{29}$, K.H. Hiller ${ }^{46}$, S.J. Hillier ${ }^{21}$, M. Hils ${ }^{48}$, I. Hinchliffe ${ }^{18}$, F. Hinterkeuser ${ }^{24}$, M. Hirose ${ }^{132}$, S. Hirose ${ }^{52}$, D. Hirschbuehl ${ }^{181}$, B. Hiti ${ }^{92}$, O. Hladik ${ }^{140}$, D.R. Hlaluku ${ }^{33 e}$, J. Hobbs ${ }^{154}$, N. Hod ${ }^{179}$, M.C. Hodgkinson ${ }^{148}$, A. Hoecker ${ }^{36}$, D. Hohn ${ }^{52}$, D. Hohov ${ }^{65}$, T. Holm ${ }^{24}$, T.R. Holmes ${ }^{37}$, M. Holzbock ${ }^{114}$, L.B.A.H. Hommels ${ }^{32}$, T.M. Hong ${ }^{138}$, J.C. Honig ${ }^{52}$, A. Hönle ${ }^{115}$, B.H. Hooberman ${ }^{172}$,

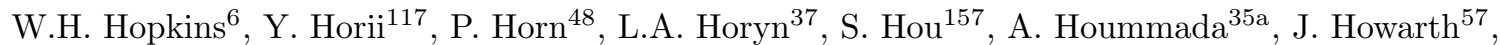
J. Hoya ${ }^{89}$, M. Hrabovsky ${ }^{130}$, J. Hrdinka ${ }^{77}$, J. Hrivnac ${ }^{65}$, A. Hrynevich ${ }^{109}$, T. Hryn'ova ${ }^{5}$, P.J. $\mathrm{Hsu}^{64}$, S.-C. $\mathrm{Hsu}^{147}$, Q. $\mathrm{Hu}^{29}$, S. $\mathrm{Hu}^{60 c}$, Y.F. Hu${ }^{15 a, 15 d, a n}$, D.P. Huang ${ }^{95}$, Y. Huang ${ }^{60 a}$, Y. Huang ${ }^{15 a}$, Z. Hubacek ${ }^{141}$, F. Hubaut ${ }^{102}$, M. Huebner ${ }^{24}$, F. Huegging ${ }^{24}$, T.B. Huffman ${ }^{134}$, M. Huhtinen ${ }^{36}$, R. Hulsken ${ }^{58}$, R.F.H. Hunter ${ }^{34}$, P. Huo ${ }^{154}$, N. Huseynov ${ }^{80, a c}$, J. Huston ${ }^{107}$, J. Huth ${ }^{59}$, R. Hyneman ${ }^{106}$, S. Hyrych ${ }^{28 a}$, G. Iacobucci ${ }^{54}$, G. Iakovidis ${ }^{29}$, I. Ibragimov ${ }^{150}$, L. Iconomidou-Fayard ${ }^{65}$, P. Iengo ${ }^{36}$, R. Ignazzi ${ }^{40}$, O. Igonkina ${ }^{120, y, *}$, R. Iguchi ${ }^{162}$, T. Iizawa ${ }^{54}$,

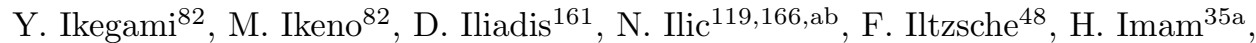

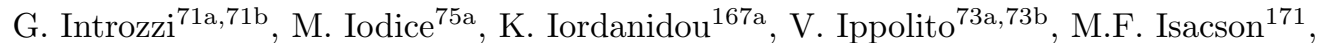

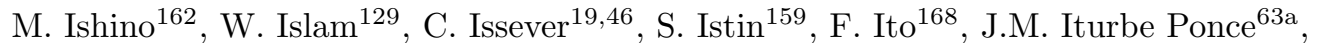

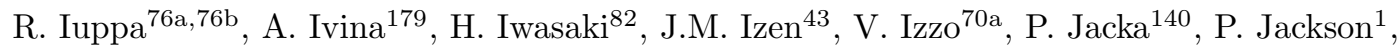
R.M. Jacobs ${ }^{46}$, B.P. Jaeger ${ }^{151}$, V. Jain ${ }^{2}$, G. Jäkel ${ }^{181}$, K.B. Jakobi ${ }^{100}$, K. Jakobs ${ }^{52}$, T. Jakoubek ${ }^{179}$, J. Jamieson ${ }^{57}$, K.W. Janas ${ }^{84 a}$, R. Jansky ${ }^{54}$, M. Janus ${ }^{53}$, P.A. Janus ${ }^{84 a}$, G. Jarlskog ${ }^{97}$, A.E. Jaspan ${ }^{91}$, N. Javadov ${ }^{80, a c}$, T. Javưrek ${ }^{36}$, M. Javurkova ${ }^{103}$, F. Jeanneau ${ }^{144}$, L. Jeanty ${ }^{131}$, J. Jejelava ${ }^{158 a}$, P. Jenni ${ }^{52, c}$, N. Jeong ${ }^{46}$, S. Jézéquel ${ }^{5}, \mathrm{H}$. Ji ${ }^{180}$, J. Jia ${ }^{154}$, H. Jiang ${ }^{79}$, Y. Jiang ${ }^{60 a}$, Z. Jiang ${ }^{152}$, S. Jiggins ${ }^{52}$, F.A. Jimenez Morales ${ }^{38}$, J. Jimenez Pena ${ }^{115}$, S. Jin ${ }^{15 c}$, A. Jinaru ${ }^{27 b}$, O. Jinnouchi ${ }^{164}$, H. Jivan ${ }^{33 e}$, P. Johansson ${ }^{148}$, K.A. Johns ${ }^{7}$, C.A. Johnson ${ }^{66}$, R.W.L. Jones ${ }^{90}$, S.D. Jones ${ }^{155}$, T.J. Jones ${ }^{91}$, J. Jongmanns ${ }^{61 a}$, J. Jovicevic ${ }^{36}$, X. Ju ${ }^{18}$, J.J. Junggeburth ${ }^{115}$, A. Juste Rozas ${ }^{14, w}$, A. Kaczmarska ${ }^{85}$, M. Kado ${ }^{73 a, 73 b}$, H. Kagan $^{127}$, M. Kagan ${ }^{152}$, A. Kahn ${ }^{39}$, C. Kahra ${ }^{100}$, T. Kaji ${ }^{178}$, E. Kajomovitz ${ }^{159}$, C.W. Kalderon ${ }^{29}$, A. Kaluza ${ }^{100}$,

A. Kamenshchikov ${ }^{123}$, M. Kaneda ${ }^{162}$, N.J. Kang ${ }^{145}$, S. Kang ${ }^{79}$, Y. Kano ${ }^{117}$, J. Kanzaki ${ }^{82}$, L.S. Kaplan ${ }^{180}$, D. Kar ${ }^{33 e}$, K. Karava ${ }^{134}$, M.J. Kareem ${ }^{167 b}$, I. Karkanias ${ }^{161}$, S.N. Karpov ${ }^{80}$, Z.M. Karpova ${ }^{80}$, V. Kartvelishvili ${ }^{90}$, A.N. Karyukhin ${ }^{123}$, A. Kastanas ${ }^{45 a, 45 b}$, C. Kato ${ }^{60 d, 60 c}$, J. Katzy ${ }^{46}$, K. Kawade ${ }^{149}$, K. Kawagoe ${ }^{88}$, T. Kawaguchi ${ }^{117}$, T. Kawamoto ${ }^{144}$, G. Kawamura ${ }^{53}$, E.F. Kay ${ }^{175}$, S. Kazakos ${ }^{14}$, V.F. Kazanin ${ }^{122 b, 122 a}$, R. Keeler ${ }^{175}$, R. Kehoe ${ }^{42}$, J.S. Keller ${ }^{34}$, E. Kellermann ${ }^{97}$, D. Kelsey ${ }^{155}$, J.J. Kempster ${ }^{21}$, J. Kendrick ${ }^{21}$, K.E. Kennedy ${ }^{39}$, O. Kepka ${ }^{140}$, S. Kersten ${ }^{181}$, B.P. Kerševan ${ }^{92}$, S. Ketabchi Haghighat ${ }^{166}$, M. Khader ${ }^{172}$, F. Khalil-Zada ${ }^{13}$, M. Khandoga ${ }^{144}$, A. Khanov ${ }^{129}$, A.G. Kharlamov ${ }^{122 b, 122 a}$, T. Kharlamova ${ }^{122 b, 122 a}$, E.E. Khoda ${ }^{174}$, A. Khodinov ${ }^{165}$, T.J. Khoo ${ }^{54}$, G. Khoriauli ${ }^{176}$, E. Khramov ${ }^{80}$, J. Khubua ${ }^{158 b}$, S. Kido ${ }^{83}$, M. Kiehn ${ }^{54}$, C.R. Kilby ${ }^{94}$, E. Kim ${ }^{164}$, Y.K. Kim ${ }^{37}$, N. Kimura ${ }^{95}$, B.T. King ${ }^{91, *}$, A. Kirchhoff ${ }^{53}$, D. Kirchmeier ${ }^{48}$, J. Kirk ${ }^{143}$, A.E. Kiryunin ${ }^{115}$, T. Kishimoto ${ }^{162}$, D.P. Kisliuk ${ }^{166}$, V. Kitali ${ }^{46}$, C. Kitsaki ${ }^{10}$, O. Kivernyk ${ }^{24}$, T. Klapdor-Kleingrothaus ${ }^{52}$, M. Klassen ${ }^{61 a}$, C. Klein ${ }^{34}$, M.H. Klein ${ }^{106}$, M. Klein ${ }^{91}$, U. Klein ${ }^{91}$, K. Kleinknecht ${ }^{100}$, P. Klimek ${ }^{121}$, A. Klimentov ${ }^{29}$, T. Klingl ${ }^{24}$, T. Klioutchnikova ${ }^{36}$, F.F. Klitzner ${ }^{114}$, P. Kluit ${ }^{120}$, S. Kluth ${ }^{115}$, E. Kneringer ${ }^{77}$, E.B.F.G. Knoops ${ }^{102}$, A. Knue ${ }^{52}$, D. Kobayashi ${ }^{88}$, T. Kobayashi ${ }^{162}$, M. Kobel ${ }^{48}$, M. Kocian ${ }^{152}$, T. Kodama ${ }^{162}$, P. Kodys ${ }^{142}$, D.M. Koeck ${ }^{155}$, P.T. Koenig ${ }^{24}$, T. Koffas ${ }^{34}$, N.M. Köhler ${ }^{36}$, 
M. Kolb ${ }^{144}$, I. Koletsou ${ }^{5}$, T. Komarek ${ }^{130}$, T. Kondo ${ }^{82}$, K. Köneke ${ }^{52}$, A.X.Y. Kong ${ }^{1}$, A.C. König ${ }^{119}$, T. Kono ${ }^{126}$, V. Konstantinides ${ }^{95}$, N. Konstantinidis ${ }^{95}$, B. Konya ${ }^{97}$,

R. Kopeliansky ${ }^{66}$, S. Koperny ${ }^{84 a}$, K. Korcyl ${ }^{85}$, K. Kordas ${ }^{161}$, G. Koren ${ }^{160}$, A. Korn ${ }^{95}$, I. Korolkov ${ }^{14}$, E.V. Korolkova ${ }^{148}$, N. Korotkova ${ }^{113}$, O. Kortner ${ }^{115}$, S. Kortner ${ }^{115}$, V.V. Kostyukhin ${ }^{148,165}$, A. Kotsokechagia ${ }^{65}$, A. Kotwal ${ }^{49}$, A. Koulouris ${ }^{10}$,

A. Kourkoumeli-Charalampidi ${ }^{71 a, 71 b}$, C. Kourkoumelis ${ }^{9}$, E. Kourlitis ${ }^{6}$, V. Kouskoura ${ }^{29}$,

R. Kowalewski ${ }^{175}$, W. Kozanecki ${ }^{101}$, A.S. Kozhin ${ }^{123}$, V.A. Kramarenko ${ }^{113}$, G. Kramberger ${ }^{92}$,

D. Krasnopevtsev ${ }^{60 a}$, M.W. Krasny ${ }^{135}$, A. Krasznahorkay ${ }^{36}$, D. Krauss ${ }^{115}$, J.A. Kremer ${ }^{100}$,

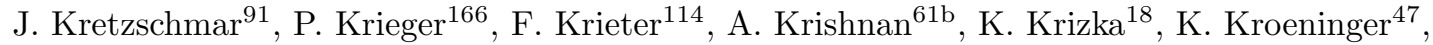
H. Kroha ${ }^{115}$, J. Kroll ${ }^{140}$, J. Kroll ${ }^{136}$, K.S. Krowpman ${ }^{107}$, U. Kruchonak ${ }^{80}$, H. Krüger ${ }^{24}$,

N. Krumnack ${ }^{79}$, M.C. Kruse ${ }^{49}$, J.A. Krzysiak ${ }^{85}$, O. Kuchinskaia ${ }^{165}$, S. Kuday ${ }^{4 b}$, J.T. Kuechler ${ }^{46}$, S. Kuehn ${ }^{36}$, A. Kugel ${ }^{61 a}$, T. Kuhl ${ }^{46}$, V. Kukhtin ${ }^{80}$, Y. Kulchitsky ${ }^{108, a e}$, S. Kuleshov ${ }^{146 b}$, Y.P. Kulinich ${ }^{172}$, M. Kuna ${ }^{58}$, T. Kunigo ${ }^{86}$, A. Kupco ${ }^{140}$, T. Kupfer ${ }^{47}$, O. Kuprash ${ }^{52}$, H. Kurashige ${ }^{83}$, L.L. Kurchaninov ${ }^{167 a}$, Y.A. Kurochkin ${ }^{108}$, A. Kurova ${ }^{112}$, M.G. Kurth ${ }^{15 a, 15 d}$, E.S. Kuwertz ${ }^{36}$, M. Kuze ${ }^{164}$, A.K. Kvam ${ }^{147}$, J. Kvita ${ }^{130}$, T. Kwan ${ }^{104}$, F. La Ruffa ${ }^{41 b, 41 a}$,

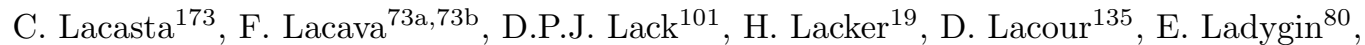
R. Lafaye $^{5}$, B. Laforge ${ }^{135}$, T. Lagouri ${ }^{146 b}$, S. Lai ${ }^{53}$, I.K. Lakomiec ${ }^{84 a}$, J.E. Lambert ${ }^{128}$,

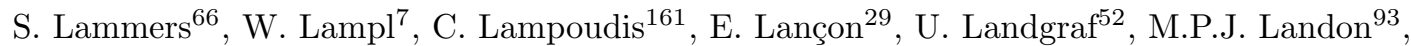
M.C. Lanfermann ${ }^{54}$, V.S. Lang ${ }^{52}$, J.C. Lange ${ }^{53}$, R.J. Langenberg ${ }^{103}$, A.J. Lankford ${ }^{170}$, F. Lanni ${ }^{29}$, K. Lantzsch ${ }^{24}$, A. Lanza ${ }^{71 a}$, A. Lapertosa ${ }^{55 b, 55 a}$, S. Laplace ${ }^{135}$, J.F. Laporte ${ }^{144}$,

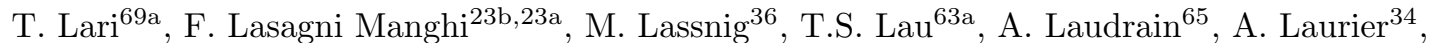
M. Lavorgna ${ }^{70 a, 70 b}$, S.D. Lawlor ${ }^{94}$, M. Lazzaroni ${ }^{69 a, 69 b}$, B. Le ${ }^{101}$, E. Le Guirriec ${ }^{102}$, A. Lebedev ${ }^{79}$, M. LeBlanc ${ }^{7}$, T. LeCompte ${ }^{6}$, F. Ledroit-Guillon ${ }^{58}$, A.C.A. Lee ${ }^{95}$, C.A. Lee ${ }^{29}$, G.R. Lee ${ }^{17}$, L. Lee ${ }^{59}$, S.C. Lee ${ }^{157}$, S. Lee ${ }^{79}$, B. Lefebvre ${ }^{167 a}$, H.P. Lefebvre ${ }^{94}$, M. Lefebvre ${ }^{175}$, C. Leggett ${ }^{18}$, K. Lehmann ${ }^{151}$, N. Lehmann ${ }^{20}$, G. Lehmann Miotto ${ }^{36}$, W.A. Leight ${ }^{46}$, A. Leisos ${ }^{161, u}$, M.A.L. Leite ${ }^{81 d}$, C.E. Leitgeb ${ }^{114}$, R. Leitner ${ }^{142}$, D. Lellouch ${ }^{179, *}$, K.J.C. Leney ${ }^{42}$, T. Lenz ${ }^{24}$, S. Leone ${ }^{72 a}$, C. Leonidopoulos ${ }^{50}$, A. Leopold ${ }^{135}$, C. Leroy ${ }^{110}$, R. Les ${ }^{166}$, C.G. Lester ${ }^{32}$, M. Levchenko ${ }^{137}$, J. Levêque ${ }^{5}$, D. Levin ${ }^{106}$, L.J. Levinson ${ }^{179}$, D.J. Lewis ${ }^{21}$, B. Li ${ }^{15 b}$, B. Li ${ }^{106}$,

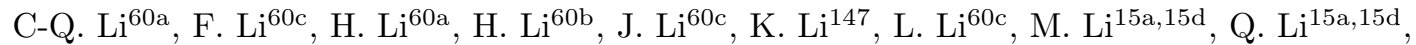

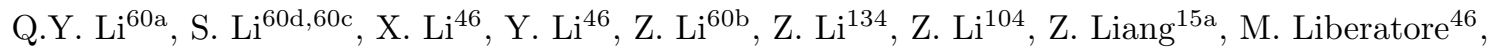
B. Liberti ${ }^{74 a}$, A. Liblong ${ }^{166}$, K. Lie ${ }^{63 c}$, S. $\operatorname{Lim}^{29}$, C.Y. $\operatorname{Lin}^{32}$, K. Lin ${ }^{107}$, R.A. Linck ${ }^{66}$, R.E. Lindley ${ }^{7}$, J.H. Lindon ${ }^{21}$, A. Linss $^{46}$, A.L. Lionti ${ }^{54}$, E. Lipeles ${ }^{136}$, A. Lipniacka ${ }^{17}$, T.M. Liss ${ }^{172, a k}$, A. Lister ${ }^{174}$, J.D. Little ${ }^{8}$, B. Liu ${ }^{79}$, B.L. Liu ${ }^{6}$, H.B. Liu ${ }^{29}$, J.B. Liu ${ }^{60 a}$,

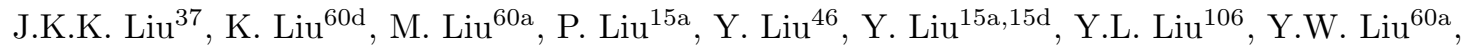
M. Livan ${ }^{71 a, 71 b}$, A. Lleres ${ }^{58}$, J. Llorente Merino ${ }^{151}$, S.L. Lloyd ${ }^{93}$, C.Y. Lo ${ }^{63 b}$, E.M. Lobodzinska ${ }^{46}$, P. Loch 7 , S. Loffredo ${ }^{74 a, 74 b}$, T. Lohse ${ }^{19}$, K. Lohwasser ${ }^{148}$, M. Lokajicek ${ }^{140}$, J.D. Long ${ }^{172}$, R.E. Long ${ }^{90}$, L. Longo ${ }^{36}$, K.A. Looper ${ }^{127}$, I. Lopez Paz ${ }^{101}$, A. Lopez Solis ${ }^{148}$, J. Lorenz ${ }^{114}$, N. Lorenzo Martinez ${ }^{5}$, A.M. Lory ${ }^{114}$, P.J. Lösel ${ }^{114}$, A. Lösle ${ }^{52}$, X. Lou ${ }^{46}$, X. Lou ${ }^{15 a}$, A. Lounis ${ }^{65}$, J. Love ${ }^{6}$, P.A. Love ${ }^{90}$, J.J. Lozano Bahilo ${ }^{173}$, M. Lu ${ }^{60 a}$, Y.J. Lu ${ }^{64}$, H.J. Lubatti ${ }^{147}$, C. Luci ${ }^{73 a, 73 b}$, F.L. Lucio Alves ${ }^{15 c}$, A. Lucotte ${ }^{58}$, F. Luehring ${ }^{66}$, I. Luise ${ }^{135}$, L. Luminari ${ }^{73 a}$, B. Lund-Jensen ${ }^{153}$,

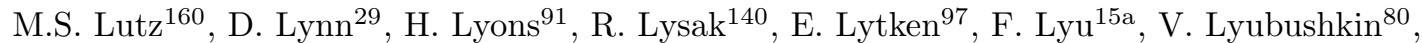
T. Lyubushkina ${ }^{80}$, H. $\mathrm{Ma}^{29}$, L.L. Ma ${ }^{60 b}$, Y. $\mathrm{Ma}^{95}$, D.M. Mac Donell ${ }^{175}$, G. Maccarrone ${ }^{51}$, A. Macchiolo ${ }^{115}$, C.M. Macdonald ${ }^{148}$, J.C. MacDonald ${ }^{148}$, J. Machado Miguens ${ }^{136}$, D. Madaffari ${ }^{173}$, R. Madar ${ }^{38}$, W.F. Mader ${ }^{48}$, M. Madugoda Ralalage Don ${ }^{129}$, N. Madysa ${ }^{48}$, J. Maeda ${ }^{83}$, T. Maeno ${ }^{29}$, M. Maerker ${ }^{48}$, V. Magerl ${ }^{52}$, N. Magini ${ }^{79}$, J. Magro67a,67c,q, D.J. Mahon ${ }^{39}$, C. Maidantchik ${ }^{81 b}$, T. Maier ${ }^{114}$, A. Maio139a,139b,139d, K. Maj ${ }^{84 a}$, O. Majersky ${ }^{28 a}$, S. Majewski ${ }^{131}$, Y. Makida ${ }^{82}$, N. Makovec ${ }^{65}$, B. Malaescu ${ }^{135}$, Pa. Malecki ${ }^{85}$, V.P. Maleev ${ }^{137}$, F. Malek ${ }^{58}$, U. Mallik ${ }^{78}$, D. Malon ${ }^{6}$, C. Malone ${ }^{32}$, S. Maltezos ${ }^{10}$, S. Malyukov ${ }^{80}$, J. Mamuzic ${ }^{173}$, 
G. Mancini ${ }^{70 a, 70 b}$, I. Mandić ${ }^{92}$, L. Manhaes de Andrade Filho ${ }^{81 a}$, I.M. Maniatis ${ }^{161}$, J. Manjarres Ramos ${ }^{48}$, K.H. Mankinen ${ }^{97}$, A. Mann ${ }^{114}$, A. Manousos ${ }^{77}$, B. Mansoulie ${ }^{144}$, I. Manthos ${ }^{161}$, S. Manzoni ${ }^{120}$, A. Marantis ${ }^{161}$, G. Marceca ${ }^{30}$, L. Marchese ${ }^{134}$, G. Marchiori ${ }^{135}$,

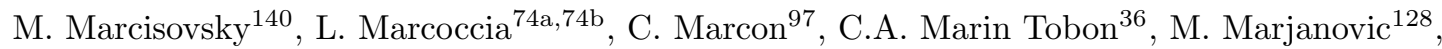
Z. Marshall ${ }^{18}$, M.U.F. Martensson ${ }^{171}$, S. Marti-Garcia ${ }^{173}$, C.B. Martin ${ }^{127}$, T.A. Martin ${ }^{177}$, V.J. Martin ${ }^{50}$, B. Martin dit Latour ${ }^{17}$, L. Martinelli ${ }^{75 a, 75 b}$, M. Martinez ${ }^{14, w}$,

P. Martinez Agullo ${ }^{173}$, V.I. Martinez Outschoorn ${ }^{103}$, S. Martin-Haugh ${ }^{143}$, V.S. Martoiu ${ }^{27 b}$, A.C. Martyniuk ${ }^{95}$, A. Marzin ${ }^{36}$, S.R. Maschek ${ }^{115}$, L. Masetti ${ }^{100}$, T. Mashimo ${ }^{162}$,

R. Mashinistov ${ }^{111}$, J. Masik ${ }^{101}$, A.L. Maslennikov122b,122a , L. Massa ${ }^{23 b, 23 a}$, P. Massarotti70a,70b, P. Mastrandrea ${ }^{72 a, 72 b}$, A. Mastroberardino ${ }^{41 b, 41 a}$, T. Masubuchi ${ }^{162}$, D. Matakias ${ }^{29}$, A. Matic ${ }^{114}$, N. Matsuzawa ${ }^{162}$, P. Mättig ${ }^{24}$, J. Maurer ${ }^{27 b}$, B. Maček ${ }^{92}$, D.A. Maximov ${ }^{122 b, 122 a}$, R. Mazini ${ }^{157}$, I. Maznas ${ }^{161}$, S.M. Mazza ${ }^{145}$, J.P. Mc Gowan ${ }^{104}$, S.P. Mc Kee ${ }^{106}$, T.G. McCarthy ${ }^{115}$, W.P. McCormack ${ }^{18}$, E.F. McDonald ${ }^{105}$, J.A. Mcfayden ${ }^{36}$, G. Mchedlidze ${ }^{158 b}$, M.A. McKay ${ }^{42}$, K.D. McLean ${ }^{175}$, S.J. McMahon ${ }^{143}$, P.C. McNamara ${ }^{105}$, C.J. McNicol ${ }^{177}$, R.A. McPherson ${ }^{175, a b}$, J.E. Mdhluli ${ }^{33 e}$, Z.A. Meadows ${ }^{103}$, S. Meehan ${ }^{36}$, T. Megy ${ }^{38}$, S. Mehlhase ${ }^{114}$, A. Mehta ${ }^{91}$,

B. Meirose ${ }^{43}$, D. Melini ${ }^{159}$, B.R. Mellado Garcia ${ }^{33 e}$, J.D. Mellenthin ${ }^{53}$, M. Melo ${ }^{28 a}$, F. Meloni ${ }^{46}$, A. Melzer ${ }^{24}$, E.D. Mendes Gouveia ${ }^{139 a, 139 e}$, L. Meng ${ }^{36}$, X.T. Meng ${ }^{106}$, S. Menke ${ }^{115}$,

E. Meoni ${ }^{41 b, 41 a}$, S. Mergelmeyer ${ }^{19}$, S.A.M. Merkt ${ }^{138}$, C. Merlassino ${ }^{134}$, P. Mermod ${ }^{54}$, L. Merola ${ }^{70 a, 70 b}$, C. Meroni ${ }^{69 a}$, G. Merz ${ }^{106}$, O. Meshkov ${ }^{113,111}$, J.K.R. Meshreki ${ }^{150}$, J. Metcalfe ${ }^{6}$, A.S. Mete $^{6}$, C. Meyer ${ }^{66}$, J-P. Meyer ${ }^{144}$, F. Miano ${ }^{155}$, M. Michetti ${ }^{19}$, R.P. Middleton ${ }^{143}$,

L. Mijović ${ }^{50}$, G. Mikenberg ${ }^{179}$, M. Mikestikova ${ }^{140}$, M. Mikuž ${ }^{92}$, H. Mildner ${ }^{148}$, M. Milesi ${ }^{105}$,

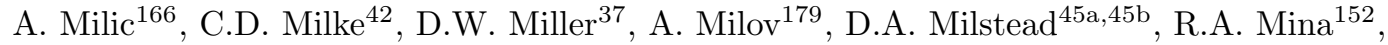
A.A. Minaenko ${ }^{123}$, I.A. Minashvili ${ }^{158 b}$, A.I. Mincer ${ }^{125}$, B. Mindur ${ }^{84 a}$, M. Mineev ${ }^{80}$, Y. Minegishi ${ }^{162}$, L.M. Mir ${ }^{14}$, M. Mironova ${ }^{134}$, A. Mirto ${ }^{68 a, 68 b}$, K.P. Mistry ${ }^{136}$, T. Mitani ${ }^{178}$, J. Mitrevski ${ }^{114}$, V.A. Mitsou ${ }^{173}$, M. Mittal ${ }^{60 c}$, O. Miu ${ }^{166}$, A. Miucci ${ }^{20}$, P.S. Miyagawa ${ }^{93}$,

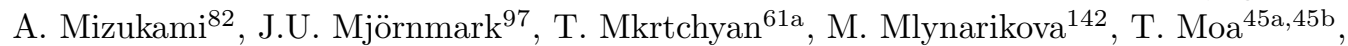
S. Mobius ${ }^{53}$, K. Mochizuki ${ }^{110}$, P. Mogg ${ }^{114}$, S. Mohapatra ${ }^{39}$, R. Moles-Valls ${ }^{24}$, K. Mönig ${ }^{46}$, E. Monnier ${ }^{102}$, A. Montalbano ${ }^{151}$, J. Montejo Berlingen ${ }^{36}$, M. Montella ${ }^{95}$, F. Monticelli ${ }^{89}$, S. Monzani ${ }^{69 a}$, N. Morange ${ }^{65}$, D. Moreno ${ }^{22 a}$, M. Moreno Llácer ${ }^{173}$, C. Moreno Martinez ${ }^{14}$, P. Morettini ${ }^{55 b}$, M. Morgenstern ${ }^{159}$, S. Morgenstern ${ }^{48}$, D. Mori ${ }^{151}$, M. Morii ${ }^{59}$, M. Morinaga ${ }^{178}$, V. Morisbak ${ }^{133}$, A.K. Morley ${ }^{36}$, G. Mornacchi ${ }^{36}$, A.P. Morris ${ }^{95}$, L. Morvaj ${ }^{154}$, P. Moschovakos ${ }^{36}$, B. $\operatorname{Moser}^{120}$, M. Mosidze ${ }^{158 b}$, T. Moskalets ${ }^{144}$, H.J. Moss ${ }^{148}$, J. Moss ${ }^{31, m}$, E.J.W. Moyse ${ }^{103}$, S. Muanza ${ }^{102}$, J. Mueller ${ }^{138}$, R.S.P. Mueller ${ }^{114}$, D. Muenstermann ${ }^{90}$, G.A. Mullier ${ }^{97}$, D.P. Mungo 69a,69b, J.L. Munoz Martinez ${ }^{14}$, F.J. Munoz Sanchez ${ }^{101}$, P. Murin ${ }^{28 b}$, W.J. Murray ${ }^{177,143}$, A. Murrone ${ }^{69 a, 69 b}$, J.M. Muse ${ }^{128}$, M. Muškinja ${ }^{18}$, C. Mwewa ${ }^{33 a}$, A.G. Myagkov ${ }^{123, a g}$, A.A. Myers ${ }^{138}$, J. Myers ${ }^{131}$, M. Myska ${ }^{141}$, B.P. Nachman ${ }^{18}$, O. Nackenhorst ${ }^{47}$, A.Nag Nag ${ }^{48}$, K. Nagai ${ }^{134}$, K. Nagano ${ }^{82}$, Y. Nagasaka ${ }^{62}$, J.L. Nagle ${ }^{29}$, E. Nagy ${ }^{102}$, A.M. Nairz ${ }^{36}$, Y. Nakahama ${ }^{117}$, K. Nakamura ${ }^{82}$, T. Nakamura ${ }^{162}$, H. Nanjo ${ }^{132}$, F. Napolitano ${ }^{61 a}$, R.F. Naranjo Garcia ${ }^{46}$, R. Narayan ${ }^{42}$, I. Naryshkin ${ }^{137}$, T. Naumann ${ }^{46}$, G. Navarro ${ }^{22 a}$, P.Y. Nechaeva ${ }^{111}$, F. Nechansky ${ }^{46}$, T.J. Neep ${ }^{21}$, A. Negri ${ }^{71 a, 71 b}$, M. Negrini ${ }^{23 b}$, C. Nellist ${ }^{119}$, C. Nelson ${ }^{104}$, M.E. Nelson ${ }^{45 a, 45 b}$, S. Nemecek ${ }^{140}$, M. Nessi ${ }^{36, e}$, M.S. Neubauer ${ }^{172}$, F. Neuhaus ${ }^{100}$, M. Neumann ${ }^{181}$, R. Newhouse ${ }^{174}$, P.R. Newman ${ }^{21}$, C.W. Ng$^{138}$, Y.S. $\mathrm{Ng}^{19}$, Y.W.Y. $\mathrm{Ng}^{170}$, B. Ngair ${ }^{35 e}$, H.D.N. Nguyen ${ }^{102}$, T. Nguyen Manh ${ }^{110}$, E. Nibigira ${ }^{38}$, R.B. Nickerson ${ }^{134}$, R. Nicolaidou ${ }^{144}$, D.S. Nielsen ${ }^{40}$, J. Nielsen ${ }^{145}$, M. Niemeyer ${ }^{53}$, N. Nikiforou ${ }^{11}$, V. Nikolaenko ${ }^{123, \text { ag }}$, I. Nikolic-Audit ${ }^{135}$, K. Nikolopoulos ${ }^{21}$, P. Nilsson ${ }^{29}$, H.R. Nindhito ${ }^{54}$,

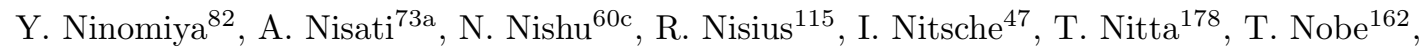
D.L. Noel ${ }^{32}$, Y. Noguchi ${ }^{86}$, I. Nomidis ${ }^{135}$, M.A. Nomura ${ }^{29}$, M. Nordberg ${ }^{36}$, J. Novak ${ }^{92}$,

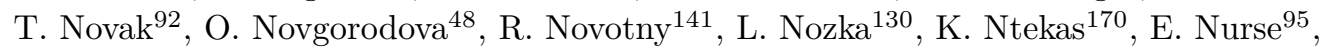


F.G. Oakham ${ }^{34, a l}$, H. Oberlack ${ }^{115}$, J. Ocariz ${ }^{135}$, A. Ochi ${ }^{83}$, I. Ochoa ${ }^{39}$, J.P. Ochoa-Ricoux ${ }^{146 a}$, K. O'Connor ${ }^{26}$, S. Oda ${ }^{88}$, S. Odaka ${ }^{82}$, S. Oerdek ${ }^{53}$, A. Ogrodnik ${ }^{84 a}$, A. Oh ${ }^{101}$, S.H. Oh ${ }^{49}$, C.C. $\mathrm{Ohm}^{153}$, H. Oide ${ }^{164}$, M.L. Ojeda ${ }^{166}$, H. Okawa ${ }^{168}$, Y. Okazaki ${ }^{86}$, M.W. O'Keefe ${ }^{91}$, Y. Okumura ${ }^{162}$, T. Okuyama ${ }^{82}$, A. Olariu ${ }^{27 b}$, L.F. Oleiro Seabra ${ }^{139 a}$, S.A. Olivares Pino ${ }^{146 a}$, D. Oliveira Damazio ${ }^{29}$, J.L. Oliver ${ }^{1}$, M.J.R. Olsson ${ }^{170}$, A. Olszewski ${ }^{85}$, J. Olszowska ${ }^{85}$, D.C. O'Neil ${ }^{151}$, A.P. O'neill ${ }^{134}$, A. Onofre ${ }^{139 a, 139 e}$, P.U.E. Onyisi ${ }^{11}$, H. Oppen ${ }^{133}$, R.G. Oreamuno Madriz ${ }^{121}$, M.J. Oreglia ${ }^{37}$, G.E. Orellana ${ }^{89}$, D. Orestano ${ }^{75 a, 75 b}$, N. Orlando ${ }^{14}$, R.S. Orr ${ }^{166}$, V. O'Shea ${ }^{57}$, R. Ospanov ${ }^{60 a}$, G. Otero y Garzon $^{30}$, H. Otono ${ }^{88}$, P.S. Ott ${ }^{61 a}$, G.J. Ottino ${ }^{18}$, M. Ouchrif ${ }^{35 d}$, J. Ouellette ${ }^{29}$, F. Ould-Saada ${ }^{133}$, A. Ouraou ${ }^{144}$, Q. Ouyang ${ }^{15 a}$, M. Owen ${ }^{57}$, R.E. Owen ${ }^{21}$, V.E. Ozcan ${ }^{12 c}$, N. Ozturk ${ }^{8}$, J. Pacalt ${ }^{130}$, H.A. Pacey ${ }^{32}$, K. Pachal ${ }^{49}$, A. Pacheco Pages ${ }^{14}$, C. Padilla Aranda ${ }^{14}$, S. Pagan Griso ${ }^{18}$, G. Palacino ${ }^{66}$, S. Palazzo ${ }^{50}$, S. Palestini ${ }^{36}$, M. Palka ${ }^{84 b}$, D. Pallin ${ }^{38}$, P. Palni ${ }^{84 a}$, C.E. Pandini ${ }^{54}$, J.G. Panduro Vazquez ${ }^{94}$, P. Pani ${ }^{46}$, G. Panizzo ${ }^{67 a, 67 c}$, L. Paolozzi ${ }^{54}$, C. Papadatos ${ }^{110}$, K. Papageorgiou ${ }^{9, g}$, S. Parajuli ${ }^{42}$, A. Paramonov ${ }^{6}$, C. Paraskevopoulos ${ }^{10}$, D. Paredes Hernandez ${ }^{63 b}$, S.R. Paredes Saenz ${ }^{134}$, B. Parida ${ }^{179}$, T.H. Park ${ }^{166}$, A.J. Parker ${ }^{31}$, M.A. Parker ${ }^{32}$, F. Parodi ${ }^{55 b, 55 a, ~ E . W . ~ P a r r i s h ~}{ }^{121}$, J.A. Parsons ${ }^{39}$, U. Parzefall ${ }^{52}$, L. Pascual Dominguez ${ }^{135}$, V.R. Pascuzzi ${ }^{18}$, J.M.P. Pasner ${ }^{145}$, F. Pasquali ${ }^{120}$, E. Pasqualucci ${ }^{73 a}$, S. Passaggio ${ }^{55 b}$, F. Pastore ${ }^{94}$, P. Pasuwan ${ }^{45 a, 45 b}$, S. Pataraia ${ }^{100}$, J.R. Pater ${ }^{101}$, A. Pathak ${ }^{180, \text { i , J. Patton }}{ }^{91}$, T. Pauly ${ }^{36}$, J. Pearkes ${ }^{152}$, B. Pearson ${ }^{115}$, M. Pedersen ${ }^{133}$, L. Pedraza Diaz ${ }^{119}$, R. Pedro ${ }^{139 a}$, T. Peiffer ${ }^{53}$, S.V. Peleganchuk ${ }^{122 b, 122 a}$, O. Penc ${ }^{140}$, H. Peng ${ }^{60 a}$, B.S. Peralva ${ }^{81 a}$, M.M. Perego ${ }^{65}$, A.P. Pereira Peixoto ${ }^{139 a}$, L. Pereira Sanchez ${ }^{45 a, 45 b}$, D.V. Perepelitsa ${ }^{29}$, E. Perez Codina ${ }^{167 a}$, F. Peri ${ }^{19}$, L. Perini ${ }^{69 a, 69 b}$, H. Pernegger ${ }^{36}$, S. Perrella ${ }^{139 a}$, A. Perrevoort ${ }^{120}$, K. Peters ${ }^{46}$, R.F.Y. Peters ${ }^{101}$, B.A. Petersen ${ }^{36}$, T.C. Petersen ${ }^{40}$, E. Petit ${ }^{102}$, A. Petridis ${ }^{1}$, C. Petridou ${ }^{161}$, P. Petroff ${ }^{65}$, F. Petrucci ${ }^{75 a, 75 b}$, M. Pettee ${ }^{182}$, N.E. Pettersson ${ }^{103}$, K. Petukhova ${ }^{142}$, A. Peyaud ${ }^{144}$, R. Pezoa ${ }^{146 d}$, L. Pezzotti ${ }^{71 a, 71 b}$, T. Pham $^{105}$, F.H. Phillips ${ }^{107}$, P.W. Phillips ${ }^{143}$, M.W. Phipps ${ }^{172}$, G. Piacquadio ${ }^{154}$, E. Pianori ${ }^{18}$, A. Picazio ${ }^{103}$, R.H. Pickles ${ }^{101}$, R. Piegaia ${ }^{30}$, D. Pietreanu ${ }^{27 b}$, J.E. Pilcher ${ }^{37}$, A.D. Pilkington ${ }^{101}$, M. Pinamonti ${ }^{67 a, 67 c}$, J.L. Pinfold ${ }^{3}$, C. Pitman Donaldson ${ }^{95}$, M. Pitt ${ }^{160}$, L. Pizzimento ${ }^{74 a, 74 b}$, M.-A. Pleier ${ }^{29}$, V. Pleskot ${ }^{142}$, E. Plotnikova ${ }^{80}$, P. Podberezko ${ }^{122 b, 122 a}$, R. Poettgen ${ }^{97}$, R. Poggi $^{54}$, L. Poggioli ${ }^{135}$, I. Pogrebnyak ${ }^{107}$, D. Pohl ${ }^{24}$, I. Pokharel ${ }^{53}$, G. Polesello ${ }^{71 a}$, A. Poley ${ }^{18}$, A. Policicchio ${ }^{73 a, 73 b}$, R. Polifka ${ }^{142}$, A. Polini ${ }^{23 b}$, C.S. Pollard ${ }^{46}$, V. Polychronakos ${ }^{29}$, D. Ponomarenko ${ }^{112}$, L. Pontecorvo ${ }^{36}$, S. Popa ${ }^{27 a}$, G.A. Popeneciu ${ }^{27 d}$, L. Portales ${ }^{5}$, D.M. Portillo Quintero ${ }^{58}$, S. Pospisil ${ }^{141}$, K. Potamianos ${ }^{46}$, I.N. Potrap ${ }^{80}$, C.J. Potter ${ }^{32}$, H. Potti ${ }^{11}$, T. Poulsen ${ }^{97}$, J. Poveda ${ }^{173}$, T.D. Powell ${ }^{148}$, G. Pownall ${ }^{46}$, M.E. Pozo Astigarraga ${ }^{36}$, P. Pralavorio ${ }^{102}$, S. Prell ${ }^{79}$, D. Price ${ }^{101}$, M. Primavera ${ }^{68 a}$, M.L. Proffitt ${ }^{147}$, N. Proklova ${ }^{112}$, K. Prokofiev ${ }^{63 c}$, F. Prokoshin ${ }^{80}$, S. Protopopescu ${ }^{29}$, J. Proudfoot ${ }^{6}$, M. Przybycien ${ }^{84 a}$, D. Pudzha ${ }^{137}$, A. Puri ${ }^{172}$, P. Puzo ${ }^{65}$, D. Pyatiizbyantseva ${ }^{112}$, J. Qian ${ }^{106}$, Y. Qin ${ }^{101}$, A. Quadt ${ }^{53}$,

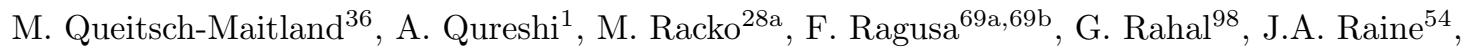
S. Rajagopalan ${ }^{29}$, A. Ramirez Morales ${ }^{93}$, K. Ran ${ }^{15 a, 15 d}$, T. Rashid ${ }^{65}$, D.M. Rauch ${ }^{46}$, F. Rauscher ${ }^{114}$, S. Rave ${ }^{100}$, B. Ravina ${ }^{148}$, I. Ravinovich ${ }^{179}$, J.H. Rawling ${ }^{101}$, M. Raymond ${ }^{36}$, A.L. $\operatorname{Read}^{133}$, N.P. Readioff ${ }^{58}$, M. Reale ${ }^{68 a, 68 b}$, D.M. Rebuzzi ${ }^{71 a, 71 b}$, G. Redlinger ${ }^{29}$, K. Reeves ${ }^{43}$, J. Reichert ${ }^{136}$, D. Reikher ${ }^{160}$, A. Reiss ${ }^{100}$, A. Rej ${ }^{150}$, C. Rembser ${ }^{36}$, A. Renardi ${ }^{46}$, M. Renda ${ }^{27 b}$, M.B. Rendel ${ }^{115}$, S. Resconi ${ }^{69 a}$, E.D. Resseguie ${ }^{18}$, S. Rettie ${ }^{95}$, B. Reynolds ${ }^{127}$, E. Reynolds ${ }^{21}$, O.L. Rezanova ${ }^{122 b, 122 a}$, P. Reznicek ${ }^{142}$, E. Ricci ${ }^{76 a, 76 b}$, R. Richter ${ }^{115}$, S. Richter ${ }^{46}$, E. Richter-Was ${ }^{84 b}$, M. Ridel ${ }^{135}$, P. Rieck ${ }^{115}$, O. Rifki ${ }^{46}$, M. Rijssenbeek ${ }^{154}$, A. Rimoldi ${ }^{71 a, 71 b}$, M. Rimoldi ${ }^{46}$, L. Rinaldi ${ }^{23 b}$, T.T. Rinn ${ }^{172}$, G. Ripellino ${ }^{153}$, I. Riu ${ }^{14}$, P. Rivadeneira ${ }^{46}$, J.C. Rivera Vergara ${ }^{175}$, F. Rizatdinova ${ }^{129}$, E. Rizvi ${ }^{93}$, C. Rizzi ${ }^{36}$, S.H. Robertson ${ }^{104, a b}$ M. Robin ${ }^{46}$, D. Robinson ${ }^{32}$, C.M. Robles Gajardo ${ }^{146 \mathrm{~d}}$, M. Robles Manzano ${ }^{100}$, A. Robson ${ }^{57}$, A. Rocchi ${ }^{74 a, 74 b}$, E. Rocco ${ }^{100}$, C. Roda ${ }^{72 a, 72 b}$, S. Rodriguez Bosca ${ }^{173}$, A.M. Rodríguez Vera ${ }^{167 b}$, 
S. Roe ${ }^{36}$, J. Roggel ${ }^{181}$, O. Røhne ${ }^{133}$, R. Röhrig ${ }^{115}$, R.A. Rojas ${ }^{146 d}$, B. Roland ${ }^{52}$, C.P.A. Roland ${ }^{66}$, J. Roloff ${ }^{29}$, A. Romaniouk ${ }^{112}$, M. Romano ${ }^{23 b, 23 a}$, N. Rompotis ${ }^{91}$, M. Ronzani ${ }^{125}$, L. Roos ${ }^{135}$, S. Rosati ${ }^{73 a}$, G. Rosin ${ }^{103}$, B.J. Rosser ${ }^{136}$, E. Rossi ${ }^{46}$, E. Rossi ${ }^{75 a}$,75b , E. Rossi ${ }^{70 a, 70 b}$,

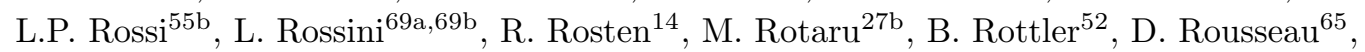
G. Rovelli71a,71b, A. Roy ${ }^{11}$, D. Roy ${ }^{33 e}$, A. Rozanov ${ }^{102}$, Y. Rozen ${ }^{159}$, X. Ruan ${ }^{33 e}$, F. Rühr ${ }^{52}$, A. Ruiz-Martinez ${ }^{173}$, A. Rummler ${ }^{36}$, Z. Rurikova ${ }^{52}$, N.A. Rusakovich ${ }^{80}$, H.L. Russell ${ }^{104}$, L. Rustige ${ }^{38,47}$, J.P. Rutherfoord ${ }^{7}$, E.M. Rüttinger ${ }^{148}$, M. Rybar ${ }^{39}$, G. Rybkin ${ }^{65}$, E.B. Rye ${ }^{133}$, A. Ryzhov ${ }^{123}$, J.A. Sabater Iglesias ${ }^{46}$, P. Sabatini ${ }^{53}$, S. Sacerdoti ${ }^{65}$, H.F-W. Sadrozinski ${ }^{145}$, R. Sadykov ${ }^{80}$, F. Safai Tehrani ${ }^{73 a}$, B. Safarzadeh Samani ${ }^{155}$, M. Safdari ${ }^{152}$, P. Saha ${ }^{121}$, S. Saha ${ }^{104}$, M. Sahinsoy ${ }^{115}$, A. Sahu ${ }^{181}$, M. Saimpert ${ }^{36}$, M. Saito ${ }^{162}$, T. Saito ${ }^{162}$, H. Sakamoto ${ }^{162}$, D. Salamani ${ }^{54}$, G. Salamanna ${ }^{75 a, 75 b}$, A. Salnikov ${ }^{152}$, J. Salt ${ }^{173}$, A. Salvador Salas ${ }^{14}$,

D. Salvatore ${ }^{41 b, 41 a}$, F. Salvatore ${ }^{155}$, A. Salvucci ${ }^{63 a, 63 b, 63 c}$, A. Salzburger ${ }^{36}$, J. Samarati ${ }^{36}$,

D. Sammel ${ }^{52}$, D. Sampsonidis ${ }^{161}$, D. Sampsonidou ${ }^{161}$, J. Sánchez ${ }^{173}$, A. Sanchez Pineda ${ }^{67 a, 36,67 c}$, H. Sandaker ${ }^{133}$, C.O. Sander ${ }^{46}$, I.G. Sanderswood ${ }^{90}$, M. Sandhoff ${ }^{181}$, C. Sandoval ${ }^{22 a}$, D.P.C. Sankey ${ }^{143}$, M. Sannino ${ }^{55 b, 55 a}$, Y. Sano ${ }^{117}$, A. Sansoni ${ }^{51}$, C. Santoni ${ }^{38}$, H. Santos ${ }^{139 a, 139 b}$, S.N. Santpur ${ }^{18}$, A. Santra ${ }^{173}$, A. Sapronov ${ }^{80}$, J.G. Saraiva ${ }^{139 a, 139 d}$, O. Sasaki ${ }^{82}$, K. Sato ${ }^{168}$, F. Sauerburger ${ }^{52}$, E. Sauvan ${ }^{5}$, P. Savard ${ }^{166, a l}$, R. Sawada ${ }^{162}$, C. Sawyer ${ }^{143}$, L. Sawyer ${ }^{96, a f}$, I. Sayago Galvan ${ }^{173}$, C. Sbarra ${ }^{23 b}$, A. Sbrizzi ${ }^{67 a, 67 c}$, T. Scanlon ${ }^{95}$, J. Schaarschmidt ${ }^{147}$, P. Schacht ${ }^{115}$, D. Schaefer ${ }^{37}$, L. Schaefer ${ }^{136}$, S. Schaepe ${ }^{36}$, U. Schäfer ${ }^{100}$, A.C. Schaffer ${ }^{65}$, D. Schaile ${ }^{114}$, R.D. Schamberger ${ }^{154}$, E. Schanet ${ }^{114}$, N. Scharmberg ${ }^{101}$, V.A. Schegelsky ${ }^{137}$, D. Scheirich ${ }^{142}$, F. Schenck ${ }^{19}$, M. Schernau ${ }^{170}$, C. Schiavi ${ }^{55 b, 55 a}$, L.K. Schildgen ${ }^{24}$, Z.M. Schillaci ${ }^{26}$, E.J. Schioppa ${ }^{68 a, 68 b}$, M. Schioppa ${ }^{41 b, 41 a}$, K.E. Schleicher ${ }^{52}$, S. Schlenker ${ }^{36}$, K.R. Schmidt-Sommerfeld ${ }^{115}$, K. Schmieden ${ }^{36}$, C. Schmitt ${ }^{100}$, S. Schmitt ${ }^{46}$, J.C. Schmoeckel ${ }^{46}$, L. Schoeffel ${ }^{144}$, A. Schoening ${ }^{61 b}$, P.G. Scholer ${ }^{52}$, E. Schopf ${ }^{134}$, M. Schott ${ }^{100}$, J.F.P. Schouwenberg ${ }^{119}$, J. Schovancova ${ }^{36}$, S. Schramm ${ }^{54}$, F. Schroeder ${ }^{181}$, A. Schulte ${ }^{100}$, H-C. Schultz-Coulon ${ }^{61 a}$, M. Schumacher ${ }^{52}$, B.A. Schumm ${ }^{145}$, Ph. Schune ${ }^{144}$, A. Schwartzman ${ }^{152}$, T.A. Schwarz ${ }^{106}$, Ph. Schwemling ${ }^{144}$, R. Schwienhorst ${ }^{107}$, A. Sciandra ${ }^{145}$, G. Sciolla ${ }^{26}$, M. Scodeggio ${ }^{46}$, M. Scornajenghi ${ }^{41 b, 41 a}$, F. Scuri ${ }^{72 a}$, F. Scutti ${ }^{105}$, L.M. Scyboz ${ }^{115}$, C.D. Sebastiani ${ }^{91}$, P. Seema ${ }^{19}$, S.C. Seidel ${ }^{118}$, A. Seiden ${ }^{145}$, B.D. Seidlitz ${ }^{29}$, T. Seiss ${ }^{37}$, C. Seitz ${ }^{46}$, J.M. Seixas ${ }^{81 b}$, G. Sekhniaidze ${ }^{70 a}$, S.J. Sekula ${ }^{42}$, N. Semprini-Cesari ${ }^{23 b, 23 a}$, S. Sen ${ }^{49}$, C. Serfon ${ }^{29}$, L. Serin ${ }^{65}$, L. Serkin ${ }^{67 a, 67 b}$, M. Sessa ${ }^{60 a}$, H. Severini ${ }^{128}$, S. Sevova ${ }^{152}$, F. Sforza ${ }^{55 b, 55 a}$, A. Sfyrla ${ }^{54}$, E. Shabalina ${ }^{53}$, J.D. Shahinian ${ }^{145}$, N.W. Shaikh ${ }^{45 a, 45 b}$, D. Shaked Renous ${ }^{179}$, L.Y. Shan ${ }^{15 a}$, M. Shapiro ${ }^{18}$, A. Sharma ${ }^{134}$, A.S. Sharma ${ }^{1}$, P.B. Shatalov ${ }^{124}$, K. Shaw ${ }^{155}$, S.M. Shaw ${ }^{101}$, M. Shehade ${ }^{179}$, Y. Shen ${ }^{128}$, A.D. Sherman ${ }^{25}$, P. Sherwood ${ }^{95}$, L. Shi ${ }^{95}$, S. Shimizu ${ }^{82}$, C.O. Shimmin ${ }^{182}$, Y. Shimogama ${ }^{178}$, M. Shimojima ${ }^{116}$, I.P.J. Shipsey ${ }^{134}$, S. Shirabe ${ }^{164}$, M. Shiyakova ${ }^{80, z}$, J. Shlomi ${ }^{179}$, A. Shmeleva ${ }^{111}$, M.J. Shochet ${ }^{37}$, J. Shojaii ${ }^{105}$, D.R. Shope ${ }^{128}$, S. Shrestha ${ }^{127}$, E.M. Shrif ${ }^{33 e}$, E. Shulga ${ }^{179}$, P. Sicho ${ }^{140}$, A.M. Sickles ${ }^{172}$, E. Sideras Haddad ${ }^{33 e}$, O. Sidiropoulou ${ }^{36}$, A. Sidoti ${ }^{23 b, 23 a}$, F. Siegert ${ }^{48}$, Dj. Sijacki ${ }^{16}$, M.Jr. Silva ${ }^{180}$, M.V. Silva Oliveira ${ }^{36}$, S.B. Silverstein ${ }^{45 a}$, S. Simion ${ }^{65}$, R. Simoniello ${ }^{100}$, C.J. Simpson-allsop ${ }^{21}$, S. Simsek ${ }^{12 b}$, P. Sinervo ${ }^{166}$, V. Sinetckii ${ }^{113}$, S. Singh ${ }^{151}$, M. Sioli ${ }^{23 b, 23 a}$, I. Siral ${ }^{131}$, S.Yu. Sivoklokov ${ }^{113}$, J. Sjölin ${ }^{45 a, 45 b}$, A. Skaf ${ }^{53}$, E. Skorda ${ }^{97}$, P. Skubic ${ }^{128}$, M. Slawinska ${ }^{85}$, K. Sliwa ${ }^{169}$, R. Slovak ${ }^{142}$, V. Smakhtin ${ }^{179}$, B.H. Smart ${ }^{143}$, J. Smiesko ${ }^{28 b}$, N. Smirnov ${ }^{112}$, S.Yu. Smirnov ${ }^{112}$, Y. Smirnov ${ }^{112}$, L.N. Smirnova ${ }^{113, r}$, O. Smirnova ${ }^{97}$, H.A. Smith ${ }^{134}$, M. Smizanska ${ }^{90}$, K. Smolek ${ }^{141}$, A. Smykiewicz ${ }^{85}$, A.A. Snesarev ${ }^{111}$, H.L. Snoek ${ }^{120}$, I.M. Snyder ${ }^{131}$, S. Snyder ${ }^{29}$, R. Sobie ${ }^{175, a b}$, A. Soffer ${ }^{160}$, A. Søgaard ${ }^{50}$, F. Sohns ${ }^{53}$, C.A. Solans Sanchez ${ }^{36}$, E.Yu. Soldatov ${ }^{112}$, U. Soldevila ${ }^{173}$, A.A. Solodkov ${ }^{123}$, A. Soloshenko ${ }^{80}$, O.V. Solovyanov ${ }^{123}$, V. Solovyev ${ }^{137}$, P. Sommer ${ }^{148}$, H. Son ${ }^{169}$, W. Song ${ }^{143}$, W.Y. Song ${ }^{167 b}$, A. Sopczak ${ }^{141}$, A.L. Sopio ${ }^{95}$, F. Sopkova ${ }^{28 b}$, S. Sottocornola ${ }^{71 a, 71 b}$, R. Soualah ${ }^{67 a, 67 c}$, A.M. Soukharev ${ }^{122 b, 122 a}$, 
D. South ${ }^{46}$, S. Spagnolo68a,68b, M. Spalla ${ }^{115}$, M. Spangenberg ${ }^{177}$, F. Spanò ${ }^{94}$, D. Sperlich ${ }^{52}$, T.M. Spieker ${ }^{61 a}$, G. Spigo ${ }^{36}$, M. Spina ${ }^{155}$, D.P. Spiteri ${ }^{57}$, M. Spousta ${ }^{142}$, A. Stabile ${ }^{69 a, 69 b}$, B.L. Stamas ${ }^{121}$, R. Stamen ${ }^{61 a}$, M. Stamenkovic ${ }^{120}$, E. Stanecka ${ }^{85}$, B. Stanislaus ${ }^{134}$, M.M. Stanitzki ${ }^{46}$, M. Stankaityte ${ }^{134}$, B. Stapf ${ }^{120}$, E.A. Starchenko ${ }^{123}$, G.H. Stark ${ }^{145}$, J. Stark ${ }^{58}$, P. Staroba ${ }^{140}$, P. Starovoitov ${ }^{61 a}$, S. Stärz ${ }^{104}$, R. Staszewski ${ }^{85}$, G. Stavropoulos ${ }^{44}$, M. Stegler ${ }^{46}$, P. Steinberg ${ }^{29}$, A.L. Steinhebel ${ }^{131}$, B. Stelzer ${ }^{151}$, H.J. Stelzer ${ }^{138}$, O. Stelzer-Chilton ${ }^{167 a}$, H. Stenzel ${ }^{56}$, T.J. Stevenson ${ }^{155}$, G.A. Stewart ${ }^{36}$, M.C. Stockton ${ }^{36}$, G. Stoicea ${ }^{27 b}$, M. Stolarski ${ }^{139 a}$, S. Stonjek ${ }^{115}$, A. Straessner ${ }^{48}$, J. Strandberg ${ }^{153}$, S. Strandberg ${ }^{45 a, 45 b}$, M. Strauss ${ }^{128}$, T. Strebler ${ }^{102}$, P. Strizenec ${ }^{28 b}$, R. Ströhmer ${ }^{176}$, D.M. Strom ${ }^{131}$, R. Stroynowski ${ }^{42}$, A. Strubig ${ }^{50}$, S.A. Stucci ${ }^{29}$, B. Stugu ${ }^{17}$, J. Stupak ${ }^{128}$, N.A. Styles ${ }^{46}$, D. Su ${ }^{152}$, W. Su ${ }^{60 c, 147}$, S. Suchek ${ }^{61 a}$, V.V. Sulin ${ }^{111}$, M.J. Sullivan ${ }^{91}$, D.M.S. Sultan ${ }^{54}$, S. Sultansoy ${ }^{4 c}$, T. Sumida ${ }^{86}$, S. Sun ${ }^{106}$, X. Sun ${ }^{101}$, K. Suruliz ${ }^{155}$, C.J.E. Suster ${ }^{156}$, M.R. Sutton ${ }^{155}$, S. Suzuki ${ }^{22}$, M. Svatos ${ }^{140}$, M. Swiatlowski ${ }^{167 a}$, S.P. Swift ${ }^{2}$, T. Swirski ${ }^{176}$, A. Sydorenko ${ }^{100}$, I. Sykora ${ }^{28 a}$, M. Sykora ${ }^{142}$, T. Sykora ${ }^{142}$, D. Ta ${ }^{100}$, K. Tackmann ${ }^{46, x}$, J. Taenzer ${ }^{160}$, A. Taffard ${ }^{170}$, R. Tafirout ${ }^{167 a}$, H. Takai ${ }^{29}$, R. Takashima ${ }^{87}$, K. Takeda ${ }^{83}$, T. Takeshita ${ }^{149}$, E.P. Takeva ${ }^{50}$, Y. Takubo ${ }^{82}$, M. Talby ${ }^{102}$, A.A. Talyshev ${ }^{122 b, 122 a}$, K.C. Tam ${ }^{63 b}$, N.M. Tamir ${ }^{160}$, J. Tanaka ${ }^{162}$, R. Tanaka ${ }^{65}$, S. Tapia Araya ${ }^{172}$, S. Tapprogge ${ }^{100}$, A. Tarek Abouelfadl Mohamed ${ }^{107}$, S. Tarem ${ }^{159}$, K. Tariq ${ }^{60 b}$, G. Tarna ${ }^{27 b, d}$, G.F. Tartarelli ${ }^{69 a}$, P. Tas ${ }^{142}$, M. Tasevsky ${ }^{140}$, T. Tashiro ${ }^{86}$, E. Tassi ${ }^{41 b, 41 a}$, A. Tavares Delgado ${ }^{139 a}$, Y. Tayalati ${ }^{35 e}$, A.J. Taylor ${ }^{50}$, G.N. Taylor ${ }^{105}$, W. Taylor ${ }^{167 b}$, H. Teagle ${ }^{91}$, A.S. Tee ${ }^{90}$, R. Teixeira De Lima ${ }^{152}$, P. Teixeira-Dias ${ }^{94}$, H. Ten Kate ${ }^{36}$, J.J. Teoh ${ }^{120}$, S. Terada ${ }^{82}$, K. Terashi ${ }^{162}$, J. Terron ${ }^{99}$, S. Terzo ${ }^{14}$, M. Testa ${ }^{51}$, R.J. Teuscher ${ }^{166, a b}$, S.J. Thais ${ }^{182}$,

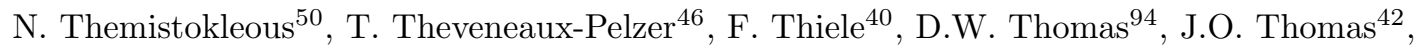
J.P. Thomas ${ }^{21}$, E.A. Thompson ${ }^{46}$, P.D. Thompson ${ }^{21}$, E. Thomson ${ }^{136}$, E.J. Thorpe ${ }^{93}$, R.E. Ticse Torres ${ }^{53}$, V.O. Tikhomirov ${ }^{111, a h}$, Yu.A. Tikhonov ${ }^{122 b, 122 a}$, S. Timoshenko ${ }^{112}$,

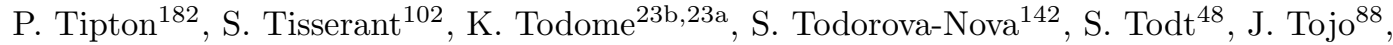
S. Tokár ${ }^{28 a}$, K. Tokushuku ${ }^{82}$, E. Tolley ${ }^{127}$, R. Tombs ${ }^{32}$, K.G. Tomiwa ${ }^{33 e}$, M. Tomoto ${ }^{117}$, L. Tompkins ${ }^{152}$, P. Tornambe ${ }^{103}$, E. Torrence ${ }^{131}$, H. Torres ${ }^{48}$, E. Torró Pastor ${ }^{147}$, C. Tosciri ${ }^{134}$, J. Toth $^{102, \text { aa }}$, D.R. Tovey ${ }^{148}$, A. Traeet $^{17}$, C.J. Treado ${ }^{125}$, T. Trefzger ${ }^{176}$, F. Tresoldi ${ }^{155}$, A. Tricoli ${ }^{29}$, I.M. Trigger ${ }^{167 a}$, S. Trincaz-Duvoid ${ }^{135}$, D.A. Trischuk ${ }^{174}$, W. Trischuk ${ }^{166}$,

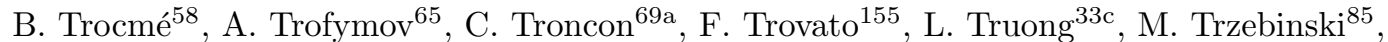

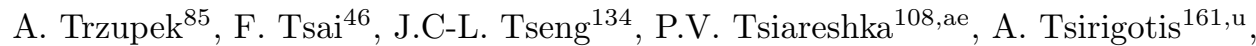
V. Tsiskaridze ${ }^{154}$, E.G. Tskhadadze ${ }^{158 a}$, M. Tsopoulou ${ }^{161}$, I.I. Tsukerman ${ }^{124}$, V. Tsulaia ${ }^{18}$,

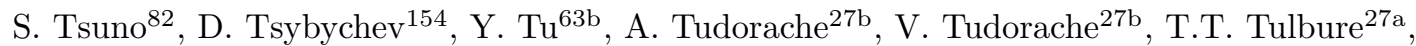
A.N. Tuna ${ }^{59}$, S. Turchikhin ${ }^{80}$, D. Turgeman ${ }^{179}$, I. Turk Cakir ${ }^{4 b, s}$, R.J. Turner ${ }^{21}$, R. Turra ${ }^{69 a}$, P.M. Tuts ${ }^{39}$, S. Tzamarias ${ }^{161}$, E. Tzovara ${ }^{100}$, K. Uchida ${ }^{162}$, F. Ukegawa ${ }^{168}$, G. Unal ${ }^{36}$,

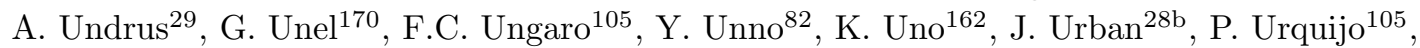

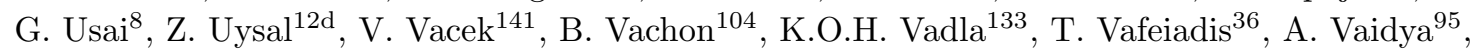
C. Valderanis ${ }^{114}$, E. Valdes Santurio ${ }^{45 a, 45 b}$, M. Valente ${ }^{54}$, S. Valentinetti ${ }^{23 b, 23 a}$, A. Valero ${ }^{173}$, L. Valéry ${ }^{46}$, R.A. Vallance ${ }^{21}$, A. Vallier ${ }^{36}$, J.A. Valls Ferrer ${ }^{173}$, T.R. Van Daalen ${ }^{14}$, P. Van Gemmeren ${ }^{6}$, I. Van Vulpen ${ }^{120}$, M. Vanadia ${ }^{74 a, 74 b}$, W. Vandelli ${ }^{36}$, M. Vandenbroucke ${ }^{144}$,

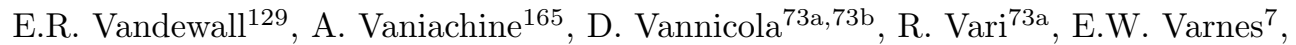
C. Varni ${ }^{55 b, 55 a}$, T. Varol ${ }^{157}$, D. Varouchas ${ }^{65}$, K.E. Varvell ${ }^{156}$, M.E. Vasile ${ }^{27 b}$, G.A. Vasquez ${ }^{175}$, F. Vazeille ${ }^{38}$, D. Vazquez Furelos ${ }^{14}$, T. Vazquez Schroeder ${ }^{36}$, J. Veatch $^{53}$, V. Vecchio ${ }^{101}$, M.J. Veen ${ }^{120}$, L.M. Veloce ${ }^{166}$, F. Veloso ${ }^{139 a, 139 c}$, S. Veneziano ${ }^{73 a}$, A. Ventura ${ }^{68 a, 68 b}$, N. Venturi ${ }^{36}$,

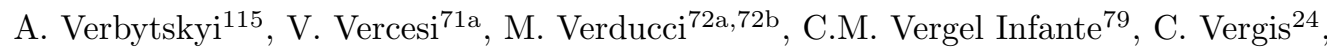
W. Verkerke ${ }^{120}$, A.T. Vermeulen ${ }^{120}$, J.C. Vermeulen ${ }^{120}$, C. Vernieri ${ }^{152}$, M.C. Vetterli ${ }^{151, \text { al }}$, N. Viaux Maira ${ }^{146 d}$, T. Vickey ${ }^{148}$, O.E. Vickey Boeriu ${ }^{148}$, G.H.A. Viehhauser ${ }^{134}$, L. Vigani ${ }^{61 b}$, M. Villa ${ }^{23 b, 23 a}$, M. Villaplana Perez $^{3}$, E.M. Villhauer ${ }^{50}$, E. Vilucchi ${ }^{51}$, M.G. Vincter ${ }^{34}$, 
G.S. Virdee ${ }^{21}$, A. Vishwakarma ${ }^{50}$, C. Vittori ${ }^{23 b, 23 a}$, I. Vivarelli ${ }^{155}$, M. Vogel ${ }^{181}$, P. Vokac $^{141}$, S.E. von Buddenbrock ${ }^{33 e}$, E. Von Toerne ${ }^{24}$, V. Vorobel $^{142}$, K. Vorobev ${ }^{112}$, M. Vos ${ }^{173}$, J.H. Vossebeld ${ }^{91}$, M. Vozak ${ }^{101}$, N. Vranjes ${ }^{16}$, M. Vranjes Milosavljevic ${ }^{16}$, V. Vrba ${ }^{141}$, M. Vreeswijk ${ }^{120}$, R. Vuillermet ${ }^{36}$, I. Vukotic ${ }^{37}$, S. Wada ${ }^{168}$, P. Wagner ${ }^{24}$, W. Wagner ${ }^{181}$, J. Wagner-Kuhr ${ }^{114}$, S. Wahdan ${ }^{181}$, H. Wahlberg ${ }^{89}$, R. Wakasa ${ }^{168}$, V.M. Walbrecht ${ }^{115}$, J. Walder ${ }^{90}$, R. Walker ${ }^{114}$, S.D. Walker ${ }^{94}$, W. Walkowiak ${ }^{150}$, V. Wallangen ${ }^{45 a, 45 b}$, A.M. Wang ${ }^{59}$, A.Z. Wang ${ }^{180}$, C. Wang ${ }^{60 c}$, F. Wang ${ }^{180}$, H. Wang ${ }^{18}$, H. Wang ${ }^{3}$, J. Wang ${ }^{63 a}$, P. Wang ${ }^{42}$, Q. Wang ${ }^{128}$, R.-J. Wang ${ }^{100}$, R. Wang ${ }^{60 a}$, R. Wang ${ }^{6}$, S.M. Wang ${ }^{157}$, W.T. Wang ${ }^{60 a}$, W. Wang ${ }^{15 c}$, W.X. Wang ${ }^{60 a}$, Y. Wang ${ }^{60 a}$, Z. Wang ${ }^{106}$, C. Wanotayaroj ${ }^{46}$, A. Warburton ${ }^{104}$, C.P. Ward ${ }^{32}$, D.R. Wardrope ${ }^{95}$, N. Warrack ${ }^{57}$, A.T. Watson ${ }^{21}$, M.F. Watson ${ }^{21}$, G. Watts ${ }^{147}$, B.M. Waugh ${ }^{95}$, A.F. Webb ${ }^{11}$, C. Weber ${ }^{29}$, M.S. Weber ${ }^{20}$, S.A. Weber ${ }^{34}$, S.M. Weber ${ }^{61 a}$, A.R. Weidberg ${ }^{134}$, J. Weingarten ${ }^{47}$, M. Weirich ${ }^{100}$, C. Weiser ${ }^{52}$, P.S. Wells ${ }^{36}$, T. Wenaus ${ }^{29}$, T. Wengler ${ }^{36}$, S. Wenig ${ }^{36}$, N. Wermes ${ }^{24}$, M. Wessels ${ }^{61 a}$, T.D. Weston ${ }^{20}$, K. Whalen ${ }^{131}$, N.L. Whallon ${ }^{147}$, A.M. Wharton ${ }^{90}$, A.S. White ${ }^{106}$, A. White ${ }^{8}$, M.J. White ${ }^{1}$, D. Whiteson ${ }^{170}$, B.W. Whitmore ${ }^{90}$, W. Wiedenmann ${ }^{180}$, C. Wiel ${ }^{48}$, M. Wielers ${ }^{143}$, N. Wieseotte ${ }^{100}$, C. Wiglesworth ${ }^{40}$, L.A.M. Wiik-Fuchs ${ }^{52}$, H.G. Wilkens ${ }^{36}$, L.J. Wilkins ${ }^{94}$, H.H. Williams ${ }^{136}$, S. Williams ${ }^{32}$, S. Willocq ${ }^{103}$, P.J. Windischhofer ${ }^{134}$, I. Wingerter-Seez ${ }^{5}$, E. Winkels ${ }^{155}$, F. Winklmeier ${ }^{131}$, B.T. Winter ${ }^{52}$, M. Wittgen ${ }^{152}$, M. Wobisch ${ }^{96}$, A. Wolf ${ }^{100}$, R. Wölker ${ }^{134}$, J. Wollrath ${ }^{52}$, M.W. Wolter ${ }^{85}$, H. Wolters ${ }^{139 a, 139 c}$, V.W.S. Wong ${ }^{174}$, N.L. Woods ${ }^{145}$, S.D. Worm ${ }^{46}$, B.K. Wosiek ${ }^{85}$, K.W. Woźniak ${ }^{85}$, K. Wraight ${ }^{57}$, S.L. Wu ${ }^{180}$, X. Wu ${ }^{54}$, Y. Wu ${ }^{60 a}$, J. Wuerzinger ${ }^{134}$, T.R. Wyatt ${ }^{101}$, B.M. Wynne ${ }^{50}$, S. Xella ${ }^{40}$, L. Xia ${ }^{177}$, J. Xiang ${ }^{63 c}$, X. Xiao ${ }^{106}$, X. Xie ${ }^{60 a}$, I. Xiotidis ${ }^{155}$, D. Xu ${ }^{15 a}$, H. Xu ${ }^{60 a}, H . \mathrm{Xu}^{60 a}$, L. Xu ${ }^{29}$, T. Xu ${ }^{144}$, W. Xu ${ }^{106}$, Z. Xu ${ }^{60 b}$, Z. Xu ${ }^{152}$, B. Yabsley ${ }^{156}$, S. Yacoob ${ }^{33 a}$, K. Yajima ${ }^{132}$, D.P. Yallup ${ }^{95}$, N. Yamaguchi ${ }^{88}$, Y. Yamaguchi ${ }^{164}$, A. Yamamoto ${ }^{82}$, M. Yamatani ${ }^{162}$, T. Yamazaki ${ }^{162}$, Y. Yamazaki ${ }^{83}$, J. Yan $^{60 c}$,

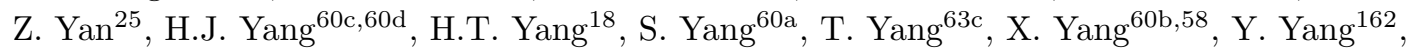

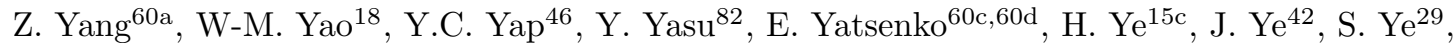

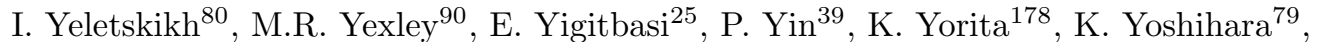
C.J.S. Young ${ }^{36}$, C. Young ${ }^{152}$, J. Yu ${ }^{79}$, R. Yuan ${ }^{60 b, h}$, X. Yue ${ }^{61 a}$, M. Zaazoua ${ }^{35 e}$, B. Zabinski ${ }^{85}$, G. Zacharis ${ }^{10}$, E. Zaffaroni ${ }^{54}$, J. Zahreddine ${ }^{135}$, A.M. Zaitsev ${ }^{123, a g}$, T. Zakareishvili ${ }^{158 b}$, N. Zakharchuk ${ }^{34}$, S. Zambito ${ }^{36}$, D. Zanzi ${ }^{36}$, D.R. Zaripovas ${ }^{57}$, S.V. Zeißner ${ }^{47}$, C. Zeitnitz ${ }^{181}$, G. Zemaityte ${ }^{134}$, J.C. Zeng ${ }^{172}$, O. Zenin ${ }^{123}$, T. Ženišs ${ }^{28 a}$, D. Zerwas ${ }^{65}$, M. Zgubič ${ }^{134}$, B. Zhang ${ }^{15 c}$, D.F. Zhang ${ }^{15 b}$, G. Zhang ${ }^{15 b}$, J. Zhang ${ }^{6}$, Kaili. Zhang ${ }^{15 a}$, L. Zhang ${ }^{15 c}$, L. Zhang ${ }^{60 a}$, M. Zhang ${ }^{172}$, R. Zhang ${ }^{180}$, S. Zhang ${ }^{106}$, X. Zhang ${ }^{60 c}$, X. Zhang ${ }^{60 b}$, Y. Zhang ${ }^{15 a, 15 d}$, Z. Zhang ${ }^{63 a}$, Z. Zhang ${ }^{65}$, P. Zhao ${ }^{49}$, Z. Zhao ${ }^{60 a}$, A. Zhemchugov ${ }^{80}$, Z. Zheng ${ }^{106}$, D. Zhong ${ }^{172}$, B. Zhou ${ }^{106}$, C. Zhou ${ }^{180}$, H. Zhou ${ }^{7}$, M.S. Zhou ${ }^{15 a, 15 d}$, M. Zhou ${ }^{154}$, N. Zhou ${ }^{60 c}$, Y. Zhou ${ }^{7}$, C.G. Zhu ${ }^{60 b}$, C. Zhu ${ }^{15 a, 15 d}$, H.L. Zhu ${ }^{60 a}$, H. Zhu ${ }^{15 a}$, J. Zhu ${ }^{106}$, Y. Zhu ${ }^{60 a}$, X. Zhuang ${ }^{15 a}$, K. Zhukov ${ }^{111}$, V. Zhulanov ${ }^{122 b, 122 a, ~}$ D. Zieminska ${ }^{66}$, N.I. Zimine ${ }^{80}$, S. Zimmermann ${ }^{52}$, Z. Zinonos ${ }^{115}$, M. Ziolkowski ${ }^{150}$, L. Živković ${ }^{16}$, G. Zobernig ${ }^{180}$, A. Zoccoli ${ }^{23 b}$,23a, K. Zoch ${ }^{53}$, T.G. Zorbas ${ }^{148}$, R. Zou ${ }^{37}$, L. Zwalinski ${ }^{36}$.

1 Department of Physics, University of Adelaide, Adelaide; Australia

2 Physics Department, SUNY Albany, Albany NY; United States of America

3 Department of Physics, University of Alberta, Edmonton AB; Canada

4 Department of Physics ${ }^{(a)}$, Ankara University, Ankara; Istanbul Aydin University ${ }^{(b)}$, Application and Research Center for Advanced Studies, Istanbul; Division of Physics ${ }^{(c)}$, TOBB University of Economics and Technology, Ankara; Turkey

5 LAPP, Université Grenoble Alpes, Université Savoie Mont Blanc, CNRS/IN2P3, Annecy; France

6 High Energy Physics Division, Argonne National Laboratory, Argonne IL; United States of America

7 Department of Physics, University of Arizona, Tucson AZ; United States of America

8 Department of Physics, University of Texas at Arlington, Arlington TX; United States of America 
9 Physics Department, National and Kapodistrian University of Athens, Athens; Greece

10 Physics Department, National Technical University of Athens, Zografou; Greece

11 Department of Physics, University of Texas at Austin, Austin TX; United States of America

12 Bahcesehir University ${ }^{(a)}$, Faculty of Engineering and Natural Sciences, Istanbul; Istanbul Bilgi University $^{(b)}$, Faculty of Engineering and Natural Sciences, Istanbul; Department of Physics ${ }^{(c)}$, Bogazici University, Istanbul; Department of Physics Engineering ${ }^{(d)}$, Gaziantep University, Gaziantep; Turkey

13 Institute of Physics, Azerbaijan Academy of Sciences, Baku; Azerbaijan

14 Institut de Física d'Altes Energies (IFAE), Barcelona Institute of Science and Technology, Barcelona; Spain

15 Institute of High Energy Physics ${ }^{(a)}$, Chinese Academy of Sciences, Beijing; Physics Department ${ }^{(b)}$, Tsinghua University, Beijing; Department of Physicss ${ }^{(c)}$, Nanjing University, Nanjing; University of Chinese Academy of Science $(U C A S)^{(d)}$, Beijing; China

16 Institute of Physics, University of Belgrade, Belgrade; Serbia

17 Department for Physics and Technology, University of Bergen, Bergen; Norway

18 Physics Division, Lawrence Berkeley National Laboratory and University of California, Berkeley CA; United States of America

19 Institut für Physik, Humboldt Universität zu Berlin, Berlin; Germany

20 Albert Einstein Center for Fundamental Physics and Laboratory for High Energy Physics, University of Bern, Bern; Switzerland

21 School of Physics and Astronomy, University of Birmingham, Birmingham; United Kingdom

22 Facultad de Ciencias y Centro de Investigaciónes ${ }^{(a)}$, Universidad Antonio Nariño, Bogotá; Departamento de Física ${ }^{(b)}$, Universidad Nacional de Colombia, Bogotá, Colombia; Colombia

23 INFN Bologna and Universita' di Bologna ${ }^{(a)}$, Dipartimento di Fisica; INFN Sezione di Bologna ${ }^{(b)}$; Italy

24 Physikalisches Institut, Universität Bonn, Bonn; Germany

25 Department of Physics, Boston University, Boston MA; United States of America

26 Department of Physics, Brandeis University, Waltham MA; United States of America

27 Transilvania University of Brasov ${ }^{(a)}$, Brasov; Horia Hulubei National Institute of Physics and Nuclear Engineering ${ }^{(b)}$, Bucharest; Department of Physics ${ }^{(c)}$, Alexandru Ioan Cuza University of Iasi, Iasi; National Institute for Research and Development of Isotopic and Molecular Technologies $^{(d)}$, Physics Department, Cluj-Napoca; University Politehnica Bucharest ${ }^{(e)}$, Bucharest; West University in Timisoara ${ }^{(f)}$, Timisoara; Romania

28 Faculty of Mathematics ${ }^{(a)}$, Physics and Informatics, Comenius University, Bratislava; Department of Subnuclear Physics ${ }^{(b)}$, Institute of Experimental Physics of the Slovak Academy of Sciences, Kosice; Slovak Republic

29 Physics Department, Brookhaven National Laboratory, Upton NY; United States of America

30 Departamento de Física, Universidad de Buenos Aires, Buenos Aires; Argentina

31 California State University, CA; United States of America

32 Cavendish Laboratory, University of Cambridge, Cambridge; United Kingdom

33 Department of Physics ${ }^{(a)}$, University of Cape Town, Cape Town; iThemba Labs ${ }^{(b)}$, Western Cape; Department of Mechanical Engineering Science ${ }^{(c)}$, University of Johannesburg, Johannesburg; University of South Africa ${ }^{(d)}$, Department of Physics, Pretoria; School of Physics ${ }^{(e)}$, University of the Witwatersrand, Johannesburg; South Africa

34 Department of Physics, Carleton University, Ottawa ON; Canada

35 Faculté des Sciences Ain Chock ${ }^{(a)}$, Réseau Universitaire de Physique des Hautes Energies Université Hassan II, Casablanca; Faculté des Sciences ${ }^{(b)}$, Université Ibn-Tofail, Kénitra; Faculté des Sciences Semlalia ${ }^{(c)}$, Université Cadi Ayyad, LPHEA-Marrakech; Faculté des Sciences ${ }^{(d)}$, Université Mohamed Premier and LPTPM, Oujda; Faculté des sciences ${ }^{(e)}$, Université Mohammed V, Rabat; Morocco

36 CERN, Geneva; Switzerland

37 Enrico Fermi Institute, University of Chicago, Chicago IL; United States of America 
40 Niels Bohr Institute, University of Copenhagen, Copenhagen; Denmark

41 Dipartimento di Fisica ${ }^{(a)}$, Università della Calabria, Rende; INFN Gruppo Collegato di Cosenza ${ }^{(b)}$, Laboratori Nazionali di Frascati; Italy

42 Physics Department, Southern Methodist University, Dallas TX; United States of America

43 Physics Department, University of Texas at Dallas, Richardson TX; United States of America

44 National Centre for Scientific Research "Demokritos", Agia Paraskevi; Greece

45 Department of Physics ${ }^{(a)}$, Stockholm University; Oskar Klein Centre ${ }^{(b)}$, Stockholm; Sweden

46 Deutsches Elektronen-Synchrotron DESY, Hamburg and Zeuthen; Germany

47 Lehrstuhl für Experimentelle Physik IV, Technische Universität Dortmund, Dortmund; Germany

48 Institut für Kern- und Teilchenphysik, Technische Universität Dresden, Dresden; Germany

49 Department of Physics, Duke University, Durham NC; United States of America

50 SUPA - School of Physics and Astronomy, University of Edinburgh, Edinburgh; United Kingdom

51 INFN e Laboratori Nazionali di Frascati, Frascati; Italy

52 Physikalisches Institut, Albert-Ludwigs-Universität Freiburg, Freiburg; Germany

53 II. Physikalisches Institut, Georg-August-Universität Göttingen, Göttingen; Germany

54 Département de Physique Nucléaire et Corpusculaire, Université de Genève, Genève; Switzerland

55 Dipartimento di Fisica ${ }^{(a)}$, Università di Genova, Genova; INFN Sezione di Genova ${ }^{(b)}$; Italy

56 II. Physikalisches Institut, Justus-Liebig-Universität Giessen, Giessen; Germany

57 SUPA - School of Physics and Astronomy, University of Glasgow, Glasgow; United Kingdom

58 LPSC, Université Grenoble Alpes, CNRS/IN2P3, Grenoble INP, Grenoble; France

59 Laboratory for Particle Physics and Cosmology, Harvard University, Cambridge MA; United States of America

60 Department of Modern Physics and State Key Laboratory of Particle Detection and Electronics ${ }^{(a)}$, University of Science and Technology of China, Hefei; Institute of Frontier and Interdisciplinary Science and Key Laboratory of Particle Physics and Particle Irradiation (MOE) ${ }^{(b)}$, Shandong University, Qingdao; School of Physics and Astronomy ${ }^{(c)}$, Shanghai Jiao Tong University, KLPPAC-MoE, SKLPPC, Shanghai; Tsung-Dao Lee Institute ${ }^{(d)}$, Shanghai; China

61 Kirchhoff-Institut für Physik ${ }^{(a)}$, Ruprecht-Karls-Universität Heidelberg, Heidelberg; Physikalisches Institut ${ }^{(b)}$, Ruprecht-Karls-Universität Heidelberg, Heidelberg; Germany

62 Faculty of Applied Information Science, Hiroshima Institute of Technology, Hiroshima; Japan

63 Department of Physics ${ }^{(a)}$, Chinese University of Hong Kong, Shatin, N.T., Hong Kong; Department of Physics ${ }^{(b)}$, University of Hong Kong, Hong Kong; Department of Physics and Institute for Advanced Study ${ }^{(c)}$, Hong Kong University of Science and Technology, Clear Water Bay, Kowloon, Hong Kong; China

64 Department of Physics, National Tsing Hua University, Hsinchu; Taiwan

65 IJCLab, Université Paris-Saclay, CNRS/IN2P3, 91405, Orsay; France

66 Department of Physics, Indiana University, Bloomington IN; United States of America

67 INFN Gruppo Collegato di Udine ${ }^{(a)}$, Sezione di Trieste, Udine; ICTP ${ }^{(b)}$, Trieste; Dipartimento Politecnico di Ingegneria e Architettura ${ }^{(c)}$, Università di Udine, Udine; Italy

68 INFN Sezione di Lecce ${ }^{(a)}$; Dipartimento di Matematica e Fisica ${ }^{(b)}$, Università del Salento, Lecce; Italy

69 INFN Sezione di Milano ${ }^{(a)}$; Dipartimento di Fisica ${ }^{(b)}$, Università di Milano, Milano; Italy

70 INFN Sezione di Napoli ${ }^{(a)}$; Dipartimento di Fisica ${ }^{(b)}$, Università di Napoli, Napoli; Italy

71 INFN Sezione di Pavia ${ }^{(a)}$; Dipartimento di Fisica ${ }^{(b)}$, Università di Pavia, Pavia; Italy

72 INFN Sezione di Pisa ${ }^{(a)}$; Dipartimento di Fisica E. Fermi ${ }^{(b)}$, Università di Pisa, Pisa; Italy

73 INFN Sezione di Roma ${ }^{(a)}$; Dipartimento di Fisica ${ }^{(b)}$, Sapienza Università di Roma, Roma; Italy

74 INFN Sezione di Roma Tor Vergata ${ }^{(a)}$; Dipartimento di Fisica ${ }^{(b)}$, Università di Roma Tor Vergata, Roma; Italy

75 INFN Sezione di Roma Tre ${ }^{(a)}$; Dipartimento di Matematica e Fisica ${ }^{(b)}$, Università Roma Tre, Roma; Italy 
76 INFN-TIFPA ${ }^{(a)}$; Università degli Studi di Trento ${ }^{(b)}$, Trento; Italy

77 Institut für Astro- und Teilchenphysik, Leopold-Franzens-Universität, Innsbruck; Austria

78 University of Iowa, Iowa City IA; United States of America

79 Department of Physics and Astronomy, Iowa State University, Ames IA; United States of America

80 Joint Institute for Nuclear Research, Dubna; Russia

81 Departamento de Engenharia Elétrica ${ }^{(a)}$, Universidade Federal de Juiz de Fora (UFJF), Juiz de Fora; Universidade Federal do Rio De Janeiro COPPE/EE/IF ${ }^{(b)}$, Rio de Janeiro; Universidade Federal de São João del Rei $(U F S J)^{(c)}$, São João del Rei; Instituto de Física ${ }^{(d)}$, Universidade de São Paulo, São Paulo; Brazil

82 KEK, High Energy Accelerator Research Organization, Tsukuba; Japan

83 Graduate School of Science, Kobe University, Kobe; Japan

84 AGH University of Science and Technology ${ }^{(a)}$, Faculty of Physics and Applied Computer Science, Krakow; Marian Smoluchowski Institute of Physicss ${ }^{(b)}$, Jagiellonian University, Krakow; Poland

85 Institute of Nuclear Physics Polish Academy of Sciences, Krakow; Poland

86 Faculty of Science, Kyoto University, Kyoto; Japan

87 Kyoto University of Education, Kyoto; Japan

88 Research Center for Advanced Particle Physics and Department of Physics, Kyushu University, Fukuoka; Japan

89 Instituto de Física La Plata, Universidad Nacional de La Plata and CONICET, La Plata; Argentina

90 Physics Department, Lancaster University, Lancaster; United Kingdom

91 Oliver Lodge Laboratory, University of Liverpool, Liverpool; United Kingdom

92 Department of Experimental Particle Physics, Jožef Stefan Institute and Department of Physics, University of Ljubljana, Ljubljana; Slovenia

93 School of Physics and Astronomy, Queen Mary University of London, London; United Kingdom

94 Department of Physics, Royal Holloway University of London, Egham; United Kingdom

95 Department of Physics and Astronomy, University College London, London; United Kingdom

96 Louisiana Tech University, Ruston LA; United States of America

97 Fysiska institutionen, Lunds universitet, Lund; Sweden

98 Centre de Calcul de l'Institut National de Physique Nucléaire et de Physique des Particules (IN2P3), Villeurbanne; France

99 Departamento de Física Teorica C-15 and CIAFF, Universidad Autónoma de Madrid, Madrid; Spain

100 Institut für Physik, Universität Mainz, Mainz; Germany

101 School of Physics and Astronomy, University of Manchester, Manchester; United Kingdom

102 CPPM, Aix-Marseille Université, CNRS/IN2P3, Marseille; France

103 Department of Physics, University of Massachusetts, Amherst MA; United States of America

104 Department of Physics, McGill University, Montreal QC; Canada

105 School of Physics, University of Melbourne, Victoria; Australia

106 Department of Physics, University of Michigan, Ann Arbor MI; United States of America

107 Department of Physics and Astronomy, Michigan State University, East Lansing MI; United States of America

108 B.I. Stepanov Institute of Physics, National Academy of Sciences of Belarus, Minsk; Belarus

109 Research Institute for Nuclear Problems of Byelorussian State University, Minsk; Belarus

110 Group of Particle Physics, University of Montreal, Montreal QC; Canada

111 P.N. Lebedev Physical Institute of the Russian Academy of Sciences, Moscow; Russia

112 National Research Nuclear University MEPhI, Moscow; Russia

113 D.V. Skobeltsyn Institute of Nuclear Physics, M.V. Lomonosov Moscow State University, Moscow; Russia

114 Fakultät für Physik, Ludwig-Maximilians-Universität München, München; Germany

115 Max-Planck-Institut für Physik (Werner-Heisenberg-Institut), München; Germany

116 Nagasaki Institute of Applied Science, Nagasaki; Japan

117 Graduate School of Science and Kobayashi-Maskawa Institute, Nagoya University, Nagoya; Japan 
Department of Physics and Astronomy, University of New Mexico, Albuquerque NM; United States of America

119 Institute for Mathematics, Astrophysics and Particle Physics, Radboud University Nijmegen/Nikhef, Nijmegen; Netherlands

120 Nikhef National Institute for Subatomic Physics and University of Amsterdam, Amsterdam; Netherlands

121 Department of Physics, Northern Illinois University, DeKalb IL; United States of America

122 Budker Institute of Nuclear Physics and NSU ${ }^{(a)}, S B$ RAS, Novosibirsk; Novosibirsk State University Novosibirsk ${ }^{(b)}$; Russia

123 Institute for High Energy Physics of the National Research Centre Kurchatov Institute, Protvino; Russia

124 Institute for Theoretical and Experimental Physics named by A.I. Alikhanov of National Research Centre "Kurchatov Institute", Moscow; Russia

125 Department of Physics, New York University, New York NY; United States of America

${ }^{126}$ Ochanomizu University, Otsuka, Bunkyo-ku, Tokyo; Japan

127 Ohio State University, Columbus OH; United States of America

128 Homer L. Dodge Department of Physics and Astronomy, University of Oklahoma, Norman OK; United States of America

129 Department of Physics, Oklahoma State University, Stillwater OK; United States of America

130 Palacký University, RCPTM, Joint Laboratory of Optics, Olomouc; Czech Republic

131 Institute for Fundamental Science, University of Oregon, Eugene, OR; United States of America

132 Graduate School of Science, Osaka University, Osaka; Japan

133 Department of Physics, University of Oslo, Oslo; Norway

134 Department of Physics, Oxford University, Oxford; United Kingdom

135 LPNHE, Sorbonne Université, Université de Paris, CNRS/IN2P3, Paris; France

136 Department of Physics, University of Pennsylvania, Philadelphia PA; United States of America

137 Konstantinov Nuclear Physics Institute of National Research Centre "Kurchatov Institute", PNPI, St. Petersburg; Russia

138 Department of Physics and Astronomy, University of Pittsburgh, Pittsburgh PA; United States of America

139 Laboratório de Instrumentação e Física Experimental de Partículas - LIP ${ }^{(a)}$, Lisboa; Departamento de Física ${ }^{(b)}$, Faculdade de Ciências, Universidade de Lisboa, Lisboa; Departamento de Física ${ }^{(c)}$, Universidade de Coimbra, Coimbra; Centro de Física Nuclear da Universidade de Lisboa $^{(d)}$, Lisboa; Departamento de Física ${ }^{(e)}$, Universidade do Minho, Braga; Departamento de Física Teórica y del $\operatorname{Cosmos}^{(f)}$, Universidad de Granada, Granada (Spain); Dep Física and CEFITEC of Faculdade de Ciências e Tecnologia ${ }^{(g)}$, Universidade Nova de Lisboa, Caparica; Instituto Superior Técnico ${ }^{(h)}$, Universidade de Lisboa, Lisboa; Portugal

140 Institute of Physics of the Czech Academy of Sciences, Prague; Czech Republic

${ }^{141}$ Czech Technical University in Prague, Prague; Czech Republic

142 Charles University, Faculty of Mathematics and Physics, Prague; Czech Republic

143 Particle Physics Department, Rutherford Appleton Laboratory, Didcot; United Kingdom

144 IRFU, CEA, Université Paris-Saclay, Gif-sur-Yvette; France

145 Santa Cruz Institute for Particle Physics, University of California Santa Cruz, Santa Cruz CA; United States of America

146 Departamento de Física ${ }^{(a)}$, Pontificia Universidad Católica de Chile, Santiago; Universidad Andres Bello $^{(b)}$, Department of Physics, Santiago; Instituto de Alta Investigación ${ }^{(c)}$, Universidad de Tarapacá; Departamento de Física ${ }^{(d)}$, Universidad Técnica Federico Santa María, Valparaíso; Chile

147 Department of Physics, University of Washington, Seattle WA; United States of America

148 Department of Physics and Astronomy, University of Sheffield, Sheffield; United Kingdom

149 Department of Physics, Shinshu University, Nagano; Japan

150 Department Physik, Universität Siegen, Siegen; Germany

151 Department of Physics, Simon Fraser University, Burnaby BC; Canada 
152 SLAC National Accelerator Laboratory, Stanford CA; United States of America

153 Physics Department, Royal Institute of Technology, Stockholm; Sweden

154 Departments of Physics and Astronomy, Stony Brook University, Stony Brook NY; United States of America

155 Department of Physics and Astronomy, University of Sussex, Brighton; United Kingdom

156 School of Physics, University of Sydney, Sydney; Australia

157 Institute of Physics, Academia Sinica, Taipei; Taiwan

158 E. Andronikashvili Institute of Physics ${ }^{(a)}$, Iv. Javakhishvili Tbilisi State University, Tbilisi; High Energy Physics Institute ${ }^{(b)}$, Tbilisi State University, Tbilisi; Georgia

159 Department of Physics, Technion, Israel Institute of Technology, Haifa; Israel

160 Raymond and Beverly Sackler School of Physics and Astronomy, Tel Aviv University, Tel Aviv; Israel

161 Department of Physics, Aristotle University of Thessaloniki, Thessaloniki; Greece

162 International Center for Elementary Particle Physics and Department of Physics, University of Tokyo, Tokyo; Japan

163 Graduate School of Science and Technology, Tokyo Metropolitan University, Tokyo; Japan

164 Department of Physics, Tokyo Institute of Technology, Tokyo; Japan

165 Tomsk State University, Tomsk; Russia

166 Department of Physics, University of Toronto, Toronto ON; Canada

167 TRIUMF ${ }^{(a)}$, Vancouver BC; Department of Physics and Astronomy ${ }^{(b)}$, York University, Toronto ON; Canada

168 Division of Physics and Tomonaga Center for the History of the Universe, Faculty of Pure and Applied Sciences, University of Tsukuba, Tsukuba; Japan

169 Department of Physics and Astronomy, Tufts University, Medford MA; United States of America

170 Department of Physics and Astronomy, University of California Irvine, Irvine CA; United States of America

171 Department of Physics and Astronomy, University of Uppsala, Uppsala; Sweden

172 Department of Physics, University of Illinois, Urbana IL; United States of America

173 Instituto de Física Corpuscular (IFIC), Centro Mixto Universidad de Valencia - CSIC, Valencia; Spain

174 Department of Physics, University of British Columbia, Vancouver BC; Canada

175 Department of Physics and Astronomy, University of Victoria, Victoria BC; Canada

176 Fakultät für Physik und Astronomie, Julius-Maximilians-Universität Würzburg, Würzburg; Germany

177 Department of Physics, University of Warwick, Coventry; United Kingdom

178 Waseda University, Tokyo; Japan

179 Department of Particle Physics, Weizmann Institute of Science, Rehovot; Israel

180 Department of Physics, University of Wisconsin, Madison WI; United States of America

181 Fakultät für Mathematik und Naturwissenschaften, Fachgruppe Physik, Bergische Universität Wuppertal, Wuppertal; Germany

182 Department of Physics, Yale University, New Haven CT; United States of America

a Also at Borough of Manhattan Community College, City University of New York, New York NY; United States of America

${ }^{b}$ Also at Centro Studi e Ricerche Enrico Fermi; Italy

c Also at CERN, Geneva; Switzerland

d Also at CPPM, Aix-Marseille Université, CNRS/IN2P3, Marseille; France

e Also at Département de Physique Nucléaire et Corpusculaire, Université de Genève, Genève; Switzerland

${ }^{f}$ Also at Departament de Fisica de la Universitat Autonoma de Barcelona, Barcelona; Spain

$g$ Also at Department of Financial and Management Engineering, University of the Aegean, Chios; Greece 
$h$ Also at Department of Physics and Astronomy, Michigan State University, East Lansing MI; United States of America

${ }^{i}$ Also at Department of Physics and Astronomy, University of Louisville, Louisville, KY; United States of America

$j$ Also at Department of Physics, Ben Gurion University of the Negev, Beer Sheva; Israel

${ }^{k}$ Also at Department of Physics, California State University, East Bay; United States of America

${ }^{l}$ Also at Department of Physics, California State University, Fresno; United States of America

${ }^{m}$ Also at Department of Physics, California State University, Sacramento; United States of America

$n$ Also at Department of Physics, King's College London, London; United Kingdom

o Also at Department of Physics, St. Petersburg State Polytechnical University, St. Petersburg; Russia

${ }^{p}$ Also at Department of Physics, University of Fribourg, Fribourg; Switzerland

$q$ Also at Dipartimento di Matematica, Informatica e Fisica, Università di Udine, Udine; Italy

$r$ Also at Faculty of Physics, M.V. Lomonosov Moscow State University, Moscow; Russia

$s$ Also at Giresun University, Faculty of Engineering, Giresun; Turkey

${ }^{t}$ Also at Graduate School of Science, Osaka University, Osaka; Japan

u Also at Hellenic Open University, Patras; Greece

$v$ Also at IJCLab, Université Paris-Saclay, CNRS/IN2P3, 91405, Orsay; France

w Also at Institucio Catalana de Recerca i Estudis Avancats, ICREA, Barcelona; Spain

$x$ Also at Institut für Experimentalphysik, Universität Hamburg, Hamburg; Germany

y Also at Institute for Mathematics, Astrophysics and Particle Physics, Radboud University Nijmegen/Nikhef, Nijmegen; Netherlands

$z$ Also at Institute for Nuclear Research and Nuclear Energy (INRNE) of the Bulgarian Academy of Sciences, Sofia; Bulgaria

aa Also at Institute for Particle and Nuclear Physics, Wigner Research Centre for Physics, Budapest; Hungary

ab Also at Institute of Particle Physics (IPP), Vancouver; Canada

ac Also at Institute of Physics, Azerbaijan Academy of Sciences, Baku; Azerbaijan

ad Also at Instituto de Fisica Teorica, IFT-UAM/CSIC, Madrid; Spain

ae Also at Joint Institute for Nuclear Research, Dubna; Russia

af Also at Louisiana Tech University, Ruston LA; United States of America

ag Also at Moscow Institute of Physics and Technology State University, Dolgoprudny; Russia

ah Also at National Research Nuclear University MEPhI, Moscow; Russia

ai Also at Physics Department, An-Najah National University, Nablus; Palestine

aj Also at Physikalisches Institut, Albert-Ludwigs-Universität Freiburg, Freiburg; Germany

ak Also at The City College of New York, New York NY; United States of America

al Also at TRIUMF, Vancouver BC; Canada

am Also at Universita di Napoli Parthenope, Napoli; Italy

an Also at University of Chinese Academy of Sciences (UCAS), Beijing; China

* Deceased 